\title{
Reliability based analysis of the crosswind stability of railway vehicles
}

\author{
vorgelegt von \\ Antonio Carrarini \\ von der Fakultät V - Verkehrs- und Maschinensysteme \\ der Technischen Universität Berlin \\ zur Erlangung des akademischen Grades \\ Doktor der Ingenieurwissenschaft (Dr.-Ing.) \\ genehmigte Dissertation
}

Promotionsausschuss:

Vorsitzender: Prof. Dr.rer.nat. V. Popov (TU Berlin)

Berichter: Prof. Dr.-Ing. M. Hecht (TU Berlin)

Prof. Dr.-Ing. C. Proppe (Universität Karlsruhe TH)

Tag der wissenschaftlichen Aussprache: 24.3.06

Berlin 2006

D 83 


\section{Danksagung}

Diese Arbeit ist während meiner Tätigkeit als wissenschaftlicher Mitarbeiter im Deutschen Zentrum für Luft- und Raumfahrt e.V. (DLR) in Oberpfaffenhofen entstanden. Mein Dank geht also zunächst an den ehemaligen Abteilungsleiter Herrn Prof. Willi Kortüm, der leider kurz nach meiner Einstellung verstarb, und an Herrn Dr. Alfred Jaschinski, der mit Hilfe von Herrn Prof. Martin Arnold die Abteilung in Abwesenheit von Herrn Kortüm leitete und mein Vorhaben unterstützte. Besonders muss ich mich bei Herrn Dr. Johann Bals bedanken, von dessen Abteilung die Gruppe Kortüm übernommen wurde; er förderte weiter das Vorhaben und schaffte die Bedingungen, um die Arbeit fertig zu stellen.

Mein bester Dank geht dann an Herrn Prof. Markus Hecht von der TU-Berlin, der als erster Gutachter die Promotion durch seine Engagement überhaupt ermöglichte und dem Thema große Aufmerksamkeit schenkte, und an Herrn Prof. Carsten Proppe von der Universität Karlsruhe, der das Koreferat übernahm und mich mit vielen sehr wertvollen Anregungen versorgt hat.

Schließlich muss ich mich bei den Mitarbeitern im DLR bedanken, von denen viele inzwischen schon in der Industrie tätig sind, die mir fachlich und menschlich zur Seite standen. Ich hoffe, selbst ein so angenehmer Kollege gewesen zu sein.

Für alle andere gilt einfach: grazie!

München, März 2006

Diese Arbeit erscheint auch beim Shaker Verlag.

This work is also published by Shaker Verlag. 


\section{Contents}

Contents

Notation and abbreviations

$\begin{array}{ll}\text { Abstract } & \text { ix }\end{array}$

Zusammenfassung $\quad$ x

1 Introduction $\quad 1$

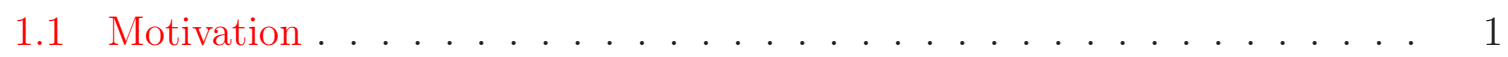

1.2 Proposed approach . . . . . . . . . . . . . . . . 2

1.3 Aim of the work . . . . . . . . . . . . . . . . . 3

1.4 State of the art . . . . . . . . . . . . . . . . 4

1.5 Structure of the work . . . . . . . . . . . . . . 6

2 Crosswind stability $\quad 8$

2.1 Crosswind stability proof . . . . . . . . . . . . . . 8

2.1.1 Overall framework ................. 8

2.1 .2 Risk analysis . . . . . . . . . . . . . . . . . 9

2.1.3 Reliability based approach . . . . . . . . . . . . . . 10

Parameters ...................... . . . 10

Risk analysis and risk assessment . . . . . . . . . . . . 11

2.2 Aerodynamics topics . . . . . . . . . . . . . . . . . . . . . . 13

2.2.1 Railway aerodynamics . . . . . . . . . . . . . . 13

Bluff bodies . . . . . . . . . . . . . . . . . . . . 14

Aerodynamic loads . . . . . . . . . . . . . . . . . . . 16

Steady aerodynamics . . . . . . . . . . . . . . 17

Unsteady aerodynamics . . . . . . . . . . . . . . 18

Nonhomogeneous flow . . . . . . . . . . . . . . . 19

2.2 .2 Wind . . . . . . . . . . . . . . . . . . . . 20

Steady wind . . . . . . . . . . . . . . . . . . . . . . . . . . . . . . . 20

Unsteady wind . . . . . . . . . . . . . . . . 21

Ideal gusts . . . . . . . . . . . . . . . . . . . . . . . . 23

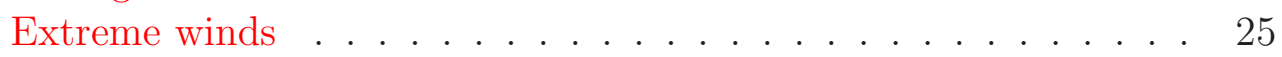

2.2 .3 Analysis tools . . . . . . . . . . . . . . . . 26

Experimental techniques . . . . . . . . . . . . . . 26

Numerical techniques . . . . . . . . . . . . . . . . . . 27 
2.3 Driving dynamics topics . . . . . . . . . . . . . . . . . . . . 27

2.3.1 Definitions . . . . . . . . . . . . . . . 27

Wheel forces . . . . . . . . . . . . . . . . . 27

Criteria and indicators for crosswind stability . . . . . . . . . 28

2.3.2 Effects of aerodynamic loads on driving dynamics . . . . . . . . 29

Steady phenomena . . . . . . . . . . . . . . 29

Unsteady phenomena . . . . . . . . . . . . . . . . . . . . . 29

2.3 .3 Analysis tools . . . . . . . . . . . . . . . . . . . . . . . . . . . . . . . . . . 30

Multibody dynamics .................... . . . 31

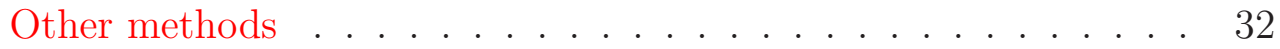

2.4 Parametric uncertainty/variability . . . . . . . . . . . . . . . . . . . . . . . . . . 33

2.4.1 Sources of uncertainty and variability . . . . . . . . . . . . . 33

2.4.2 Models for uncertainty . . . . . . . . . . . . . . . . . . . 33

2.4.3 Stochastic variables . . . . . . . . . . . . . . . . 34

Dual representation . . . . . . . . . . . . . . 36

3 Modelling and simulation $\quad 38$

3.1 Vehicle model . . . . . . . . . . . . . . . . . . . . . . . . . . . 39

3.1 .1 Mechanical model . . . . . . . . . . . . . . . . . . . 39

Multibody model . . . . . . . . . . . . . . . . . . 39

Multibody model without W/R contact . . . . . . . . . . . 40

Linear model . . . . . . . . . . . . . . . . . . . . . . . . . . . . . . . . 42

3.1.2 Aerodynamic model of the vehicle . . . . . . . . . . . . . . . . . . . . 42

Aerodynamic coefficients . . . . . . . . . . . . . 43

Statistics of the aerodynamic coefficients . . . . . . . . . 43

References ...................... . . 44

3.2 Environment model . . . . . . . . . . . . . . . . . . . . 46

3.2.1 Track scenario . . . . . . . . . . . . . . . . . 46

3.2.2 Aerodynamic model of the environment . . . . . . . . . . . . . 46

Gust shape . . . . . . . . . . . . . . . . . 47

Gust amplitude (gust factor) . . . . . . . . . . . . . . . . . . 48

Gust length (duration) . . . . . . . . . . . . . . . . . . . . 49

Final crosswind scenario . . . . . . . . . . . . 50

3.3 Simulation . . . . . . . . . . . . . . . . . . 51

4 Reliability evaluation $\quad 53$

4.1 Basic concepts . . . . . . . . . . . . . . . . . . . . . . . . . . 54

4.1 .1 Probability of failure . . . . . . . . . . . . . 54

4.1 .2 Limit state . . . . . . . . . . . . . . . . . 56

4.1.3 Limit state for the crosswind stability . . . . . . . . . . . . . 57

4.2 Evaluation of the probability of failure . . . . . . . . . . . . . . . . . . . . . . . 58

4.2.1 Semi-analytical techniques . . . . . . . . . . . . . . 58

Mean value . . . . . . . . . . . . . . . . . . . . 58

First order reliability method . . . . . . . . . . . . . 59 
MPP locus . . . . . . . . . . . . . . . . . . 63

4.2.2 Nonnormal and correlated variates . . . . . . . . . . 66

Rosenblatt transformation . . . . . . . . . . . . 66

Rackwitz-Fiessler (R-F) . . . . . . . . . . . . . . 66

Orthogonal transformation . . . . . . . . . . . . . . . 68

Comparison . . . . . . . . . . . . . . . . . . 70

4.2.3 Sampling based techniques . . . . . . . . . . . . . . . 70

Monte Carlo . . . . . . . . . . . . . . . . . . . . . 70

Accuracy and variance reduction . . . . . . . . . . . . 72

4.2.4 Other tools . . . . . . . . . . . . . . . . . . . . 73

Metamodels .................... . . 73

Screening . . . . . . . . . . . . . . . . 75

4.2.5 Comparison of the methods . . . . . . . . . . . . 76

4.3 Probabilistic sensitivity analysis . . . . . . . . . . . . . 76

4.3 .1 Reliability sensitivity . . . . . . . . . . . . . . . 77

FORM approach . . . . . . . . . . . . . . . 77

Sampling based approach . . . . . . . . . . . . . 80

4.3 .2 Response sensitivity . . . . . . . . . . . . . . . . . 81

Uncertainty propagation . . . . . . . . . . . . 81

Variance based sensitivity . . . . . . . . . . . . . . . 82

4.4 Optimisation . . . . . . . . . . . . . . . . 86

4.4.1 Optimisation and risk analysis . . . . . . . . . . . 86

4.4 Multiobjective optimisation . . . . . . . . . . . . . . . 87

4.4.3 Reliability based optimisation . . . . . . . . . . . . . . . . 88

4.4.4 Robust crosswind stability . . . . . . . . . . . . . . . 89

$\begin{array}{lll}5 & \text { Results } & 91\end{array}$

5.1 Straight track . . . . . . . . . . . . . . . . . . . 91

5.1.1 Probabilistic characteristic wind curve . . . . . . . . . . . 91

5.1 .2 Sensitivity . . . . . . . . . . . . . . . . . 91

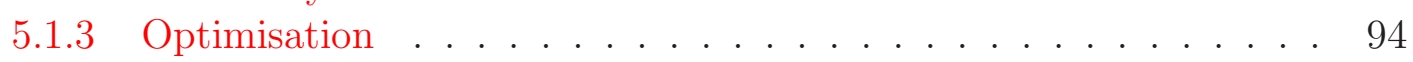

5.2 Curve . . . . . . . . . . . . . . . . . . . . . . . . 94

5.2.1 General results . . . . . . . . . . . . . . . . . . 94

5.2 .2 Characteristic wind curves . . . . . . . . . . . . . . . 97

6 Summary and conclusions 99

6.1 Main results and contributions . . . . . . . . . . . . . . . . . . . 99

6.2 Suggestions . . . . . . . . . . . . . . . . . . . . 100

6.3 Further work . . . . . . . . . . . . . . . . . 101

$\begin{array}{ll}\text { Bibliography } & 109\end{array}$ 


\section{Notation and abbreviations}

Having given precedence to the use of the standard notation in each of the different fields addressed in this work, some incongruences and overlappings in the notation could not be avoided.

Vectors and matrices are bold.

In nondeterministic context:

$A, B \quad$ stochastic variates

$a, b \quad$ outcome of stochastic variates $\mid$ deterministic parameters

$\hat{a}, \hat{b} \quad$ Monte Carlo estimates

$a_{0}, b_{0} \quad$ nominal (design) value of uncertain/variable parameters

Greek letters:

$\alpha \quad$ direction cosine

$\beta \quad$ reliability index

$\delta \quad$ wind angle | Kronecker's delta

$\phi \quad$ normal probability distribution function | roll angle

$\Phi \quad$ normal cumulative distribution function

$\gamma \quad$ nick angle

$\mu \quad$ expected value

$\Omega \quad$ failure region

$\rho \quad$ correlation coefficient | air density

$\sigma \quad$ standard deviation

$\theta \quad$ yaw angle (wind)

Latin letters:

$\begin{array}{ll}A & \text { reference area } \\ c & \text { track cant | constraint of the optimisation task } \\ c_{d} & \text { cant deficiency } \\ C & \text { aerodynamic coefficient }{ }^{1} \\ \mathrm{E}[\cdot] & \text { expected value } \\ F & \text { cumulative distribution function } \mid \text { aerodynamic load }\end{array}$

${ }^{1}$ When not otherwise noted, the aerodynamic coefficients refer to a yaw angle $\theta=20^{\circ}$. 


\begin{tabular}{|c|c|}
\hline$f$ & probability distribution function \\
\hline$g$ & limit state function in the parameter space \\
\hline$g^{\prime}$ & limit state function in the gaussian space \\
\hline GustF & gust factor \\
\hline Gust $\Delta t$ & gust duration \\
\hline$h$ & importance sampling distribution \\
\hline$H$ & aerodynamic admittance function \\
\hline$M_{X}$ & aerodynamic roll moment \\
\hline$M_{Y}$ & aerodynamic pitch moment \\
\hline$M_{Z}$ & aerodynamic yaw moment \\
\hline$n$ & number of system parameters/variables $(=$ dimension of $\mathbf{X}$ ) \\
\hline$N$ & number of Monte Carlo samples \\
\hline$p$ & generic parameter influencing the reliability index $\beta$ \\
\hline$p_{F}$ & probability of failure \\
\hline$Q$ & $\mathrm{~W} / \mathrm{R}$ vertical force (wheel load) \\
\hline$Q^{\prime}$ & wheel unloading \\
\hline$Q_{L}^{\prime}$ & limit for the wheel unloading \\
\hline$r$ & curve radius \\
\hline$R$ & risk \\
\hline$s$ & rail span \\
\hline$S$ & power spectral density \\
\hline$S_{i j \ldots}$ & sensitivity index \\
\hline$t$ & time \\
\hline$T$ & transformation for correlated variates \\
\hline$U$ & wind speed | normal variate \\
\hline$U_{0}$ & mean (background) wind speed \\
\hline$U_{r}$ & relative wind velocity \\
\hline$V$ & driving velocity \\
\hline $\operatorname{Var}[\cdot]$ & variance \\
\hline$V_{i j \ldots} \ldots$ & factor of the variance decomposition \\
\hline$X$ & uncertain/variable system parameter (stochastic variable) \\
\hline$X^{\prime}$ & reduced variables $\left(\mu_{X}=0, \sigma_{X}=1\right)$ \\
\hline $\bar{x}$ & deterministic design parameter \\
\hline$x^{*}$ & coordinate of the MPP \\
\hline$x^{\prime *}$ & coordinate of the MPP in the reduced space \\
\hline $\mathbf{x}_{\sim i j \ldots}$ & vector $\mathbf{x}$ rid of the $\mathrm{i}$-th, $\mathrm{j}$-th, $\ldots$ elements \\
\hline$Y$ & $\begin{array}{l}\text { aerodynamic side force } \mid \mathrm{W} / \mathrm{R} \text { lateral force } \mid \text { transformed } \\
\text { variable (via } T \text { ) }\end{array}$ \\
\hline$Z$ & aerodynamic lift force \\
\hline
\end{tabular}




$\begin{array}{ll}\text { Abbreviations: } \\ \text { CDF } & \text { cumulative distribution function } \\ \text { CFD } & \text { computational fluid dynamics } \\ \text { CWC } & \text { characteristic wind curve } \\ \text { DB } & \text { major German railway company } \\ \text { DLR } & \text { German aerospace centre } \\ \text { DOF } & \text { degree of freedom } \\ \text { EOG } & \text { extreme operational gust } \\ \text { FORM } & \text { first order reliability method }{ }^{2} \\ \text { ICE } & \text { German high speed trains family } \\ \text { IS } & \text { importance sampling } \\ \text { MBS } & \text { multibody system, multibody dynamics } \\ \text { MC } & \text { Monte Carlo } \\ \text { MOPS } & \text { optimisation package developed at the DLR } \\ \text { MPP } & \text { most probable (failure) point } \\ \text { MV } & \text { mean value method } \\ \text { PCWC } & \text { probabilistic characteristic wind curve } \\ \text { PDF } & \text { probability distribution function } \\ \text { RIL401 } & \text { German guideline for crosswind stability issued by DB } \\ \text { RS } & \text { response surface } \\ \text { SIMPACK } & \text { multibody simulation software originally developed at the DLR } \\ \text { TSI } & \text { technical specifications of interoperability (European norms) } \\ \text { W/R } & \text { wheel/rail }\end{array}$

\footnotetext{
${ }^{2}$ This designation, and the corresponding acronym, are commonly used in practice but actually not correct, see for example [DM96].

${ }^{3}$ The part dealing with crosswind stability (EN 14067-4) is meant.
} 


\section{Abstract}

Keywords: crosswind stability, railway vehicles, driving dynamics, reliability analysis

Nowadays, crosswind stability is a key topic for the homologation of railway vehicles and thus a pivotal boundary condition in their design process. In many countries, including Germany, the safety proof is based on the numerical simulation of the driving behaviour of the vehicle in extreme situations and must necessarily include the aerodynamic and driving performances of the vehicle as well as the wind conditions to be reasonably expected during operation. It follows that the quality of the safety proof depends on the accuracy of the available models. In this respect a deep gap can be observed: on the one hand high accuracy can be reached by multibody simulation in the investigation of the driving dynamics; on the other hand the aerodynamic loads acting on the vehicle, which depend on the vehicle shape and the wind scenario, can be estimated only with poor accuracy. The latter problem is due to the difficulties in the set up of models and the determination of the system parameters because of the complexity of the three dimensional flow around the vehicle and the implicit stochastic nature of atmospheric wind. In the present norms for crosswind stability such modelling uncertainties are usually not considered or are very empirically taken into account by safety factors.

In this work some improvement in the modelling of the aerodynamic phenomena and the driving dynamics are firstly introduced. It could be observed that with regard to aerodynamics more complex models than usual are necessary, e.g. to cover unsteady phenomena; on the contrary, simpler models than usual can be sufficient for the analysis of the driving dynamics, allowing, for example, the use of linear system theory. Then, in order to include parametric uncertainty in the safety proof, the conventional risk analysis for crosswind stability has been coupled with methods from reliability analysis. Such methods, which are quite common in structural mechanics and are available in different formulations, lead to the efficient assessment of the risk and thus to a reduction of the safety factors, provided that a statistical description of the uncertainties is available. Even though the provision of such a description is often a challenge, good results can be also achieved on the basis of little available information. Furthermore, sensitivity analysis and optimisation can be reformulated on the basis of the proposed approach, so that reliability analysis can be integrated not only in the safety proof but also in the design process. The discussed methods have been tested on the real case of a German high speed train. 


\section{Zusammenfassung}

Schlüsselwörter: Seitenwindstabilität, Schienenfahrzeuge, Fahrdynamik, Zuverlässigkeitsanalyse

Die Seitenwindstabilität spielt heutzutage eine entscheidende Rolle im Zulassungsverfahren von Schienenfahrzeugen und wird oft zur zentralen Randbedingung im Entwurfsprozess. In vielen Ländern, einschließlich Deutschland, basiert der Sicherheitsnachweis auf der numerischen Simulation des Fahrverhaltens unter ausgewählten Randbedingungen und enthält sowohl die fahrdynamischen und aerodynamischen Eigenschaften des Fahrzeugs als auch die zu erwartenden Windverhältnisse. Die Güte des Nachweises hängt deshalb von der Genauigkeit der verfügbaren Berechnungsmodelle ab. In dieser Hinsicht ist eine große Diskrepanz festzustellen: mittels der Mehrkörperdynamik ist es einerseits möglich, bei bekannten Randbedingungen das Verhalten des fahrenden Fahrzeugs sehr genau zu ermitteln; andererseits können die auf das Fahrzeug wirkenden aerodynamischen Lasten, die von der aerodynamischen Gestaltung des Fahrzeugs und dem Windszenario abhängen, nur grob geschätzt werden. Die Ursache liegt in der Komplexität der Strömung um das Fahrzeug und dem stochastischen Charakter des atmosphärischen Windes, die die Erstellung von Modellen und die Ermittlung der Werte der Systemparameter erschweren. In den Normen zur Seitenwindstabilität bleiben solche parametrischen Unsicherheiten grundsätzlich unberücksichtigt bzw. sie werden mit empirischen Sicherheitsfaktoren behandelt.

In dieser Arbeit werden zunächst Verbesserungen in der Modellierung erarbeitet, sowohl auf der aerodynamischen als auch der fahrzeugdynamischen Seite. Es ergibt sich, dass im ersteren Fall komplexe Modelle unbedingt notwendig sind, um z.B. instationäre aerodynamische Vorgänge abzubilden, während im letzteren Fall einfachere Modelle als die üblichen kompletten Mehrkörpermodelle ausreichend wären und zum Beispiel die Anwendung der linearen Systemanalyse erlauben würden. Um die parametrischen Unsicherheiten in den Sicherheitsnachweis einzubeziehen, wird dann in dieser Arbeit die konventionelle Risikoanalyse mit Methoden aus der Zuverlässigkeitsanalyse gekoppelt. Solche Methoden, die in verschiedenen Formulierungen verfügbar sind und breite Anwendung in der Strukturmechanik finden, führen zur Quantifizierung des Risikos und erlauben deshalb letztendlich eine Reduktion der notwendigen Sicherheitsfaktoren. Dabei wird vorausgesetzt, dass eine statistische Beschreibung der Unsicherheiten gegeben ist. Obwohl die Erfüllung dieser Bedingung eine große Herausforderung darstellt, können wertvolle Aussagen auch bei minimaler verfügbarer Information gemacht werden. Darüber hinaus werden Sensitivitätsanalyse und Optimierung entsprechend dem vorgestellten Ansatzes formuliert und erweitert, um die Anwendung der Zuverlässigkeitsanalyse nicht nur im Nachweis- sondern auch im Entwurfsprozess zu ermöglichen. Die vorgestellten Methoden werden auf den realistischen Fall eines Hochgeschwindigkeitszugs angewandt. 


\section{Introduction}

\subsection{Motivation}

In the last years the crosswind stability of railway vehicles has been object of large interest by research and industry. EU projects on this topic were carried out and norms have been or are being compiled in different countries as well as on international basis. One reason for this attention can be found in the dramatic evolution performed in these years by railway transportation systems. Three characteristics of modern vehicles that significantly influence the crosswind stability can be pointed out:

- Lightweight construction increases the technical and economic efficiency of the vehicles. On the other hand, the force of gravity is the only restoring force which can prevent the train from overturning in cross winds.

- High driving velocities allow shorter journey times. On the other hand, the relative oncoming flow velocity and thus the related aerodynamic loads increase.

- Distributed traction increases the traction performances. On the other hand, the first car, which is the most endangered by aerodynamic loads, is no more a heavy locomotive but a comparatively lighter car.

Even though the main attention is often given to high-speed transportation, crosswind stability is a relevant topic also for freight vehicles, because of their low tare weight, and for slow but very light vehicles exposed to high winds, like those operating on railway lines in the Alps. A fundamental difference between such systems and high-speed transportation is that, for the former, a precautionary service interruption is bearable and thus solutions based on wind velocity monitoring along the track are of interest. For the latter, on the contrary, service interruption has to be avoided and, even though wind monitoring is still a possible aid, "all-weather" operation is striven for. This can mainly be achieved by appropriate design of the vehicles and track fencing.

As a matter of fact, crosswind stability has become one of the most critical items in the approval procedure of rolling stock and thus also a crucial boundary condition in the design process of new vehicles. Frequently the only possible solution to insufficient crosswind stability, even though in many respects uneconomic and counterproductive, is to provide the vehicles with ballast in the underbelly or in the bogies.

Today, the proof of the crosswind stability is usually the result of a process based on a more or less complex risk analysis. This process will be summarised and discussed in Sec. 2; at this point, only the main features must be stressed: 


\section{Introduction}

- The risk analysis is based on the computation of the driving behaviour (typically multibody simulations). The vehicle is assumed to drive in an ideal track/wind scenario which should represent the most endangering situation encountered during real operation (worst case).

- All the parameters (aerodynamic coefficients and mechanical parameters of the vehicle, parameters describing the scenario, etc.) used in the computations are assumed to be correct and unvarying, i.e. deterministic.

These features were and still are object of criticism. Except for the fact that computations are used, which is at the present day the only reasonable way to tackle the problem, the use of an ideal worst case scenario instead of closer to reality, possibly measured, environmental data is questionable. Moreover, even accepting that a representative ideal scenario can be identified, the assumption that the parameters are exact and fixed is an oversimplification. Firstly, natural wind is a stochastic process which cannot be modelled satisfactory in a deterministic way. Secondly, some of the parameters of the system, in particular the aerodynamic coefficients, are difficult to measure and are consequently known with a relatively low level of accuracy; their values can also be affected by large fluctuations because are influenced by factors, like turbulence intensity, that are not easy to handle.

The main drawbacks arising from the conventional deterministic approach are:

- over-conservative limits are produced, because the risk analysis is based on a poor modelling of reality, and at the same time large potential risk regions remain unchecked;

- little information suitable to suggest improvements in the design of future vehicles and to guide efforts for better modelling is provided.

Finally, it must be noted that the problem of parametric uncertainty is intrinsically considered in every norm by the introduction of safety margins. For example, the RIL401 norm sets a safety margin of $5 \%$ for the wheel unloading and explicitly justifies this choice referring to the uncertainties in the aerodynamic and mechanical parameters of the computational models.

\subsection{Proposed approach}

In this work the uncertain/variable parameters of the crosswind stability analysis are modelled as stochastic variables and managed by well established methods from the reliability analysis. In Fig. 1.1 the basic components of this approach are summarised and compared with the conventional approach; a complete description will be given in Sec. 2.1. The proposed approach will be tested on a German ICE2 driving trailer.

The main steps of the analysis process are:

- Definition of the parameters. Deterministic parameters are substituted by stochastic variables to account for uncertainty and variability. A probabilistic description of the parameters must be available as an input for the process. 


\subsection{Aim of the work}

- Computation. In the conventional approach series of simulations are performed ("parametric analysis") whereas in the proposed approach the simulations are organised in a reliability analysis framework, which is necessary to manage the uncertainties.

- Analysis of the results. In the conventional approach deterministic limits are obtained, clearly separating the safe from the unsafe vehicle behaviour. On the contrary, the output of the reliability based approach are probabilities of failure, which are finally used to compute the overall risk.

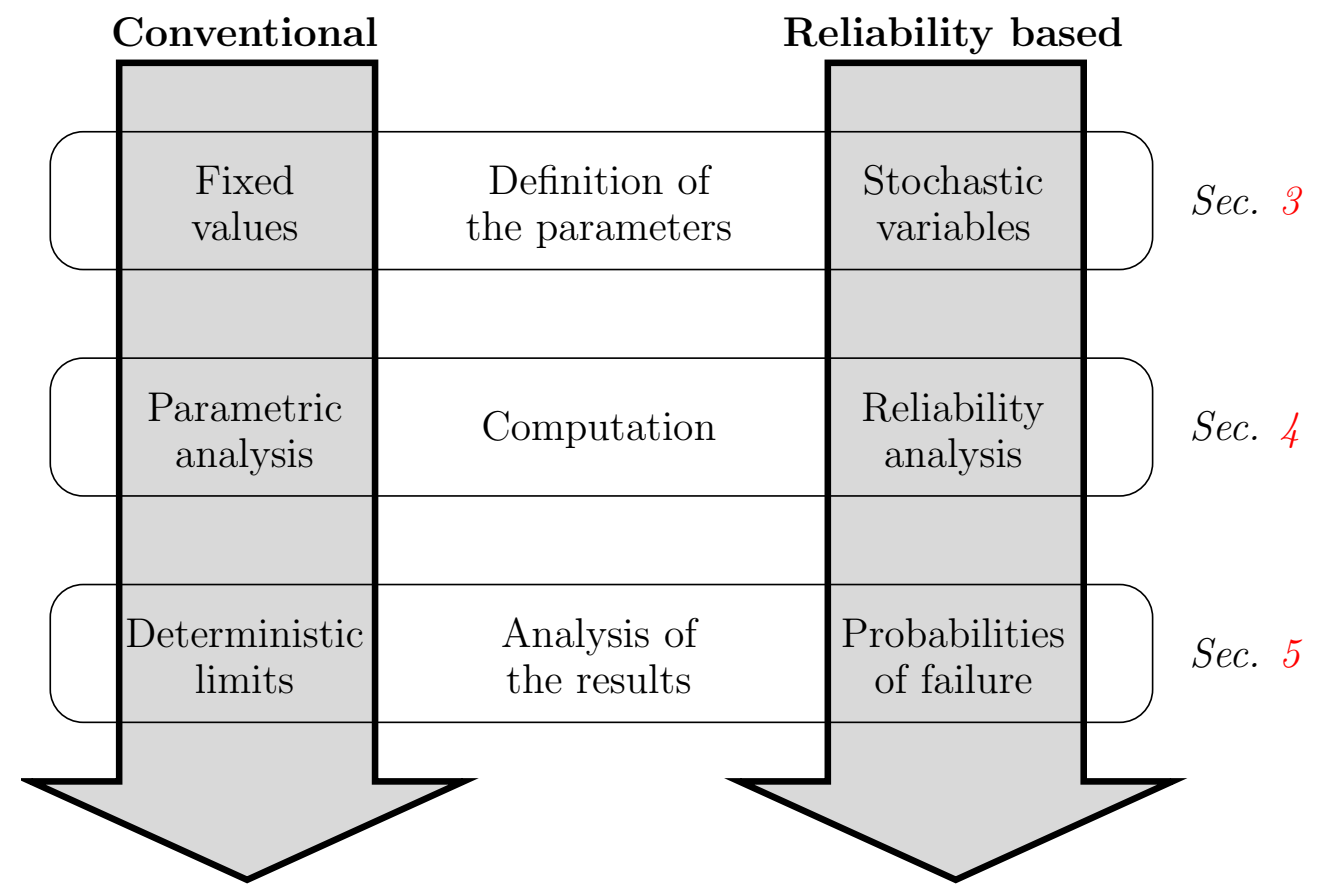

Figure 1.1: Comparison between the conventional (left) and the proposed reliability based (right) approach to the crosswind stability, with references to the sections in this work.

\subsection{Aim of the work}

The aim of the work is to outline a methodology for a closer to reality assessment of the crosswind induced risk by considering the uncertainty and variability in the parameters of the computational models. This methodology should be capable to be integrated in the corresponding crosswind stability norms, as has been done for example in structural engineering, where norms are largely based on considerations on parametric uncertainty. Not only more sensible risk assessment and thus better founded safety is to be expected, but also a general increase of operative limits, as the need for empirical safety margins is reduced. 


\section{Introduction}

The downside of the improvement are the computational effort and the large amount of input required (statistical description of the uncertainties). The former point is not an hurdle, as computational power becomes continuously cheaper. On the contrary, the latter is still a critical issue because it calls for among others for expensive and technically challenging aerodynamic experiments. However, the costs presently induced by the crosswind stability question (operative limitations, wind fencing, ballasting of vehicles, etc.) justify almost any effort.

At the same time, aim of this work is to address special aspects of the crosswind stability task that are usually given little attention. The most important is the unsteady aerodynamics topic, which can have dramatic influence on the risk assessment and is strongly connected to the choice of a representative risk scenario (ideal gust). Secondly, linear vehicle models, derived directly and automatically from the complete multibody model, would allow, with acceptable computational effort, a very efficient risk assessment using, for example, real measured wind scenarios in time or frequency domain. Finally, sensitivity and optimisation are considered, because, even though not directly required for the crosswind stability proof, they are the most important tools in the design process of new vehicles.

It must be stressed that in this work topics are addressed which stem from different engineering fields: driving dynamics, aerodynamics, probability and statistics, structural mechanics, wind engineering. None of these topics has been worked out up to its state-ofthe-art but the problem of the crosswind stability has been tackled in a multidisciplinar way, not focusing on the single aspects but on the question in its completeness. As a matter of fact, the crosswind stability issue can only be managed efficiently by the cooperation of many specialists. This work also aims to give an overview on a possible framework for such a cooperation.

\subsection{State of the art}

Historical remarks In Japan, because of the risk of typhoons and the large diffusion of narrow gauge tracks, crosswind induced accidents are reported systematically starting from the end of the XIX century and anemometers on exposed locations were already used around 1930, [Sai02]. On the contrary, the interest for the crosswind stability topic in Europe is relatively recent. Even though some wind induced accidents at the beginning of the XX century are reported, [Gaw94], and aerodynamic loads arising from asymmetric inflow were investigated in wind tunnel already in the 50s, e.g. [Ibi53], the attention raised with the diffusion of high-speed transportation in the 80s, [NS76, Coo79, Sac83]. The definitive impulse was given by the introduction of Electrical Multiple Units, which led to the substitution of locomotives with driving trailers and motor coaches. A large European research project on the theme, TRANSAERO, [SW+ 02], run in the late 90s and the first guideline dealing with the crosswind stability topic in Germany, the RIL401, [MGB02], was issued in 2000. Finally, the section of the Technical Specification of Interoperability (TSI) dealing with the crosswind stability, [CEN05], is expected to be approved in 2005 . 
Reliability analysis Reliability analysis is a well established engineering field, mainly in connection with structural mechanics, [MKL86, DM96, Mel99, Rac01]. In the last decade the topic has gained larger attention also for its suitability to robust design and design under uncertainties, [PSP93, OSR98, DC00]. As a matter of fact many computer codes are available which deal with reliability analysis and related topics (Isight, Nessus, Dakota, MSC.Robust Design, etc.). Nonetheless, the application of reliability analysis techniques to the risk analysis arising from the crosswind stability of vehicles is a new issue. This can be explained observing what follows:

- For road vehicles the crosswind stability task does not lead to a reliability problem because the main attention is on the driver's control; risk is only relevant for high sided vehicles like buses and lorries. In this field two works have to be cited: in [Bak94] extensive series of computations are performed to map the crosswind stability of cars for ranges of driver model parameters and then quantify the risk; in [SS98] reliability techniques are applied to the crosswind stability task of buses to analyse, a posteriori, occurred accidents.

- For railway vehicles the risk analysis connected with the crosswind stability can be well managed by reliability analysis, as was discussed in the previous sections. However, the problem has emerged only recently and limited efforts have been made yet; moreover, problems related to railway transportation are given in general far less attention than those related to road transportation ${ }^{1}$.

Lastly, it must be stressed that approaches to the reliability analysis exist which are not based on a modelling of the uncertain parameters as stochastic variables. To be mentioned are interval based, [PG02, DS03], and, more recently, fuzzy based techniques, [MB04].

Crosswind stability As the crosswind stability is a relatively young topic, not much research work has been done yet. The reference is defined by the corresponding norms or guidelines, where they exist; in Germany it is at the moment the RIL401, [MGB02]. A good overview of norms and standards in different countries can be found in [Lip99], where modelling and computational aspects are focused. In [AHSS04] more recent developments are reported, covering the whole risk assessment process. In France special effort was recently done for the double deck (and thus high endangered) TGV Duplex as well as for the very exposed TGV Mediterranée line, [RR01, D $\left.{ }^{+} 03\right]$. A good overview on ongoing work, with special attention on UK, can also be found in [BS03].

All the current approaches are based on a scheme that is mainly unchanged since its first formulation in [Coo79]. In this context only one basic assumption of this scheme must be pointed out: the parameters which determine the overturning wind velocity are deterministic and thus a deterministic Characteristic Wind Curve is defined and used in the subsequent risk assessment. This fact, which will be discussed in Sec. 2.1.1, represents in sum the state of the art for the topics handled in this work.

\footnotetext{
${ }^{1}$ This is obvious considering for example that in Germany at the moment more than $80 \%$ of the passenger movements is covered by individual road transportation and less than $10 \%$ by railway transportation.
} 


\section{Introduction}

\subsection{Structure of the work}

The structure of the work can be grasped referring to the general framework of the crosswind stability depicted in Fig. 1.2. In such diagram, which will be referenced and explained in the next sections, the whole framework is included; however, this work focuses on the risk analysis, which is the core of the overall process. The content of each chapters can be summarised as follows:

Ch. 2 The crosswind stability is presented and the risk analysis framed and discussed as a basis for the following explanation of the proposed reliability based approach. The two conventional building blocks of the process, aerodynamics (including both vehicle and environmental aerodynamics) and driving dynamics, are then critically reviewed; the third and new block due to the proposed approach, namely the parametric uncertainty/variability, is finally introduced.

Ch. 3 The computational model (vehicle and environment) and the simulation process are presented. Special attention is given to unsteady aerodynamic effects, which are neglected in the present norms, and to alternative modelling strategies for the driving dynamics part.

Ch. 4 Basic concepts of reliability analysis and different techniques for the reliability evaluation are described. Some features of sensitivity analysis and optimisation in the reliability framework are also introduced. In this chapter the methodologies are exemplified by application to the complete model but considering only one driving velocity.

Ch. 5 Selected results for the whole operative driving velocity range, mainly in form of Characteristic Wind Curves, are reported.

Ch. 6 After the final discussion suggestions and indications for further work are given. 


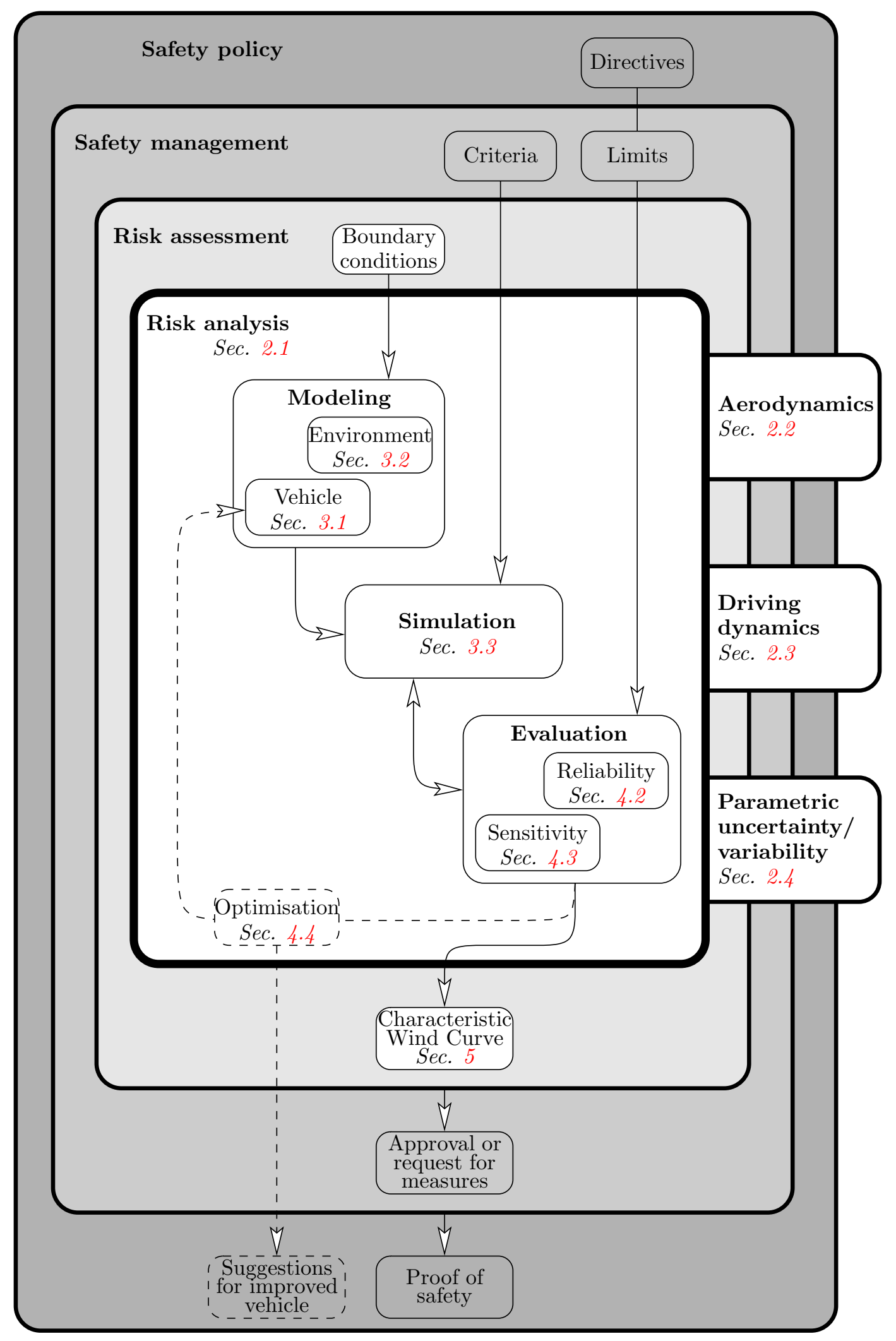

Figure 1.2: Overall framework of the crosswind stability with references to the sections in this work, which focuses on the risk analysis. 


\section{Crosswind stability}

\section{Chapter overview}

This work copes with the risk analysis, which is the part of the overall crosswind stability assessment process that deals directly with the driving dynamics. The risk analysis establishes a computational model of the vehicle and the environment and assesses under which conditions the vehicle overturns (or reaches the stability limits).

In this chapter the risk analysis task is formulated and its elements are introduced and discussed: aerodynamics and driving dynamics for the crosswind stability in general, and parametric uncertainty/variability for the proposed reliability based approach. At the same time a critical review is carried on throughout the chapter, corroborating on the one hand the need for the proposed approach and, on the other hand, suggesting general improvements to the usual models, from which also the conventional deterministic approach could benefit.

\subsection{Crosswind stability proof}

\subsubsection{Overall framework}

The typical framework of the crosswind stability is depicted in Fig. 1.2 on page 7. Although the structure of Fig. 1.2 is very general and representative, a universally accepted denomination for the components of the framework does not exist; this work follows the conventions used in [Bun96a, Bun96b]. The framework consists of four nested levels:

- The outmost level is the safety policy, which is fulfilled by the Parliaments or high political institutions. At this level directives are enacted defining the tolerability of risk up to concrete values (e.g. accidents for Mkm, [Rai02]).

- The second level is called safety management and is under the responsibility of national railway bureaus and railway companies. The risk is identified, analysed and described and indications for the criteria to be used (e.g. wheel unloading) are given. At the same time the directives from the safety policy level are converted into concrete limits (e.g. value of the acceptable wheel unloading).

- The risk assessment denotes the quantification of the risk for a specific vehicle on a specific connection (track and timetable); this is in practice the level of the norms. The track is firstly divided into sections with homogeneous characteristics: curved or straight track, exposition to wind, expected relative wind direction, expected 


\subsection{Crosswind stability proof}

maximal wind velocity in one year, etc. Many different methods can then be used to assess the risk; for example, according to the German RIL401 a kind of cumulative risk over the whole line for the vehicle under examination is compared to the risk corresponding to a reference vehicle that is assumed to be safe.

- Finally, the risk analysis is the module which determines if the vehicle drives safely under given boundary conditions, i.e. which crosswind velocity leads to the limit wheel unloading for the vehicle driving on a generic environment corresponding to the given conditions.

Once the risk analysis has been performed and the crosswind stability performances of the vehicle are known, the process recursively goes through the levels, see Fig. 1.2. Firstly, the risk is quantified in the risk assessment. This leads, if the specifications are met, to the approval by the safety management board; otherwise measures like track fencing, modifications of the time table or even modification of the vehicle (ballasting) are requested. The iteration is continued until the safety management approves the connection and the proof of safety can be issued.

\subsubsection{Risk analysis}

The risk analysis has been defined as the core of the risk assessment. However, it must be seen in practice as a pre-processing module, mapping a priori the behaviour of the vehicle for all reasonable boundary conditions, not only those actually needed for the risk assessment. This is to say, the risk analysis is performed only once in the life of a vehicle to establish the CWC, which can be seen as a part of the vehicle's data sheet; many risk assessments are then performed, usually at different times, for every line on which the train is going to operate.

The risk analysis is composed of three main steps, see Fig. 1.2 :

- Modeling. A wide range of computational model are used or have been proposed. As to the vehicle, a multibody model is usually used for higher driving velocities. For the environment, ideal track and a deterministic (ideal) wind gust are usually used.

- Simulation. The behaviour of the vehicle is simulated to estimate the indicators of the safety criteria. According to the model this step can be e.g. a time integration or the solution of an equation system.

- Evaluation. The response is finally evaluated to check if and to which extent the safety limits are exceeded. In the approach proposed in this work the evaluation is generalised by a reliability analysis. The sensitivity of the system is also evaluated for a possible use in an optimisation loop.

With regard to simulation and evaluation, it has to be emphasised that in the conventional deterministic approach, simulations covering the whole operative range are first performed and then the risk is evaluated; the evaluation consists only in checking 


\section{Crosswind stability}

whether a given limit is exceeded or not. On the contrary, in the reliability based approach the scenarios to be simulated cannot be set a priori but only in the course of the analysis according to the reliability evaluation algorithm. In fact such algorithms are, for nonlinear systems, always iterative and cause an interdependency between simulation and evaluation, see Sec. 4. This is depicted in Fig. 1.2 be means of a bidirectional connection linking the simulation and evaluation blocks.

The final output of the risk analysis is the Characteristic Wind Curve (CWC), which reports for every driving velocity the crosswind velocity which can be borne by the vehicle, i.e. the wind velocity for which the indicators (e.g. wheel unloading) reach their limit. Equivalently the curve can be interpreted as the maximal safe driving velocity for every given crosswind velocity or, more in general, as the limit between safe and failure region. In practice there are many curves for a vehicle, referring to different conditions which influence the driving dynamics, mainly straight track and curved track with different cant deficiencies. However, track quality and other parameters can be considered too. It must be finally observed that the definition of the wind velocity can be problematic. At this moment it is only important to stress that the wind velocity referenced in the characteristic wind curve is the mean (also background) wind velocity $U_{0}$ and not the gust peak velocity. More details will be given in Sec. 3 .

\subsubsection{Reliability based approach}

In the deterministic approach the crosswind stability is influenced, for given vehicle and track conditions, only by the driving velocity $V$ and the wind velocity $U_{0}$ because all system parameters are assumed to have certain fixed values. It follows that the risk analysis has only to check for every couple $\left(U_{0}, V\right)$ if the vehicle drives safely, the result of this parametric study being the CWC. Then, the risk assessment uses the CWC to compute the risk on a specific track with specific meteorological conditions and a specific timetable.

In practice, the system parameters are affected by uncertainties and variability, which are normally neglected or managed by empirical safety limits. Thus, in the proposed approach the uncertainties in the parameters are modelled and their effects are quantified by reliability analysis techniques. The main concept behind these techniques is that, when a system is affected by uncertainties in its parameters, it is not possible to determine if it is safe or unsafe under given conditions and only a probability of failure $p_{F}$ can be evaluated. A formal definition of $p_{F}$ will be given in Sec. 4 , where the methods for its evaluation are also presented.

\section{Parameters}

In the crosswind stability, the parameters which are mainly affected by uncertainty are the aerodynamic coefficients of the vehicle and the parameters that describe the ideal gust (gust amplitude and gust duration, for a given gust shape). They will be discussed in details in Sec. 3. In the reliability based approach such parameters are modelled as stochastic variables and denoted as $X_{i}$. These parameters exist only in the context of 


\subsection{Crosswind stability proof}

the risk analysis which aims to check if the vehicle drives safely for every couple $\left(U_{0}, V\right)$ and in the presence of the uncertainties. The relationship between risk analysis and assessment is discussed more deeply in the next subsection, see Fig. 2.1.

The wind velocity $U_{0}$ must be given special attention. In the risk analysis $U_{0}$ is a deterministic parameter: the general crosswind stability performance of the vehicle is checked considering every possible (reasonable) atmospheric condition i.e. every value of $U_{0}$. On the contrary, $U_{0}$ is a stochastic variable in the risk assessment, where the crosswind stability is checked for a specific track for which real atmospheric conditions and thus statistics for the wind velocity $U_{0}$, possibly not constant along the track, are considered, see Table 2.1.

Table 2.1: Stochastic and deterministic parameters arising in the risk analysis and in the risk assessment.

\begin{tabular}{c|c||c|c}
\multicolumn{2}{c||}{ parameters } & $\begin{array}{c}\text { risk } \\
\text { analysis }\end{array}$ & $\begin{array}{c}\text { risk } \\
\text { assessment }\end{array}$ \\
\hline \hline $\begin{array}{c}X_{i} \\
i=1 . . n\end{array}$ & $\begin{array}{c}\text { aerodinamic coefficients, } \\
\text { gust amplitude and duration }\end{array}$ & stochastic & - \\
\hline$U_{0}$ & mean wind velocity & deterministic & stochastic \\
\hline & driving velocity & deterministic & deterministic
\end{tabular}

In this work it is assumed that the statistics of the gust parameters are independent from the mean wind velocity $U_{0}$. This assumption is not always admissible but simplifies the reliability analysis and does not influence the general validity of the approach.

\section{Risk analysis and risk assessment}

To better comprehend the probabilistic approach to the crosswind stability the risk analysis, which is the proper subject of this work, must be framed in the risk assessment. In what follows, a homogeneous scenario is assumed, i.e. the properties of the track and of the environment as well as the driving velocity are supposed to be constant along the track. This is equivalent to considering only a track section with constant properties.

The process is structured as follows, see Fig. 2.1:

- The core is the reliability analysis, which computes the probability of failure $p_{F}$ as a function of the stochastic parameters $\mathbf{X}$ (aerodynamic coefficients of the 


\section{Crosswind stability}

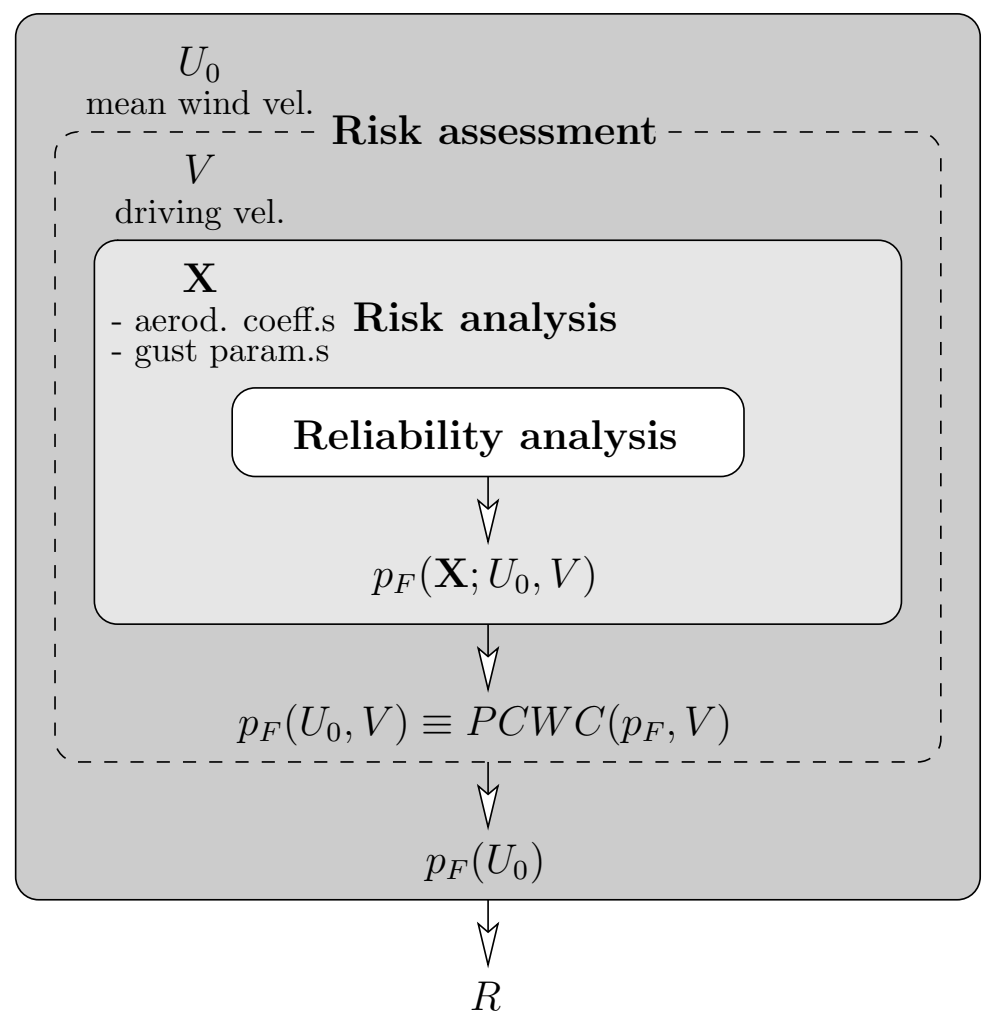

Figure 2.1: Risk analysis and assessment.

vehicle and gust parameters), the driving velocity $V$ and the mean wind velocity $U_{0}$ :

$$
p_{F}=p_{F}\left(\mathbf{X} ; U_{0}, V\right) .
$$

In the reliability analysis the driving velocity and the mean wind velocity are deterministic parameters and thus not directly involved in the reliability evaluation. This is to say, the reliability analysis for the stochastic parameters $\mathbf{X}$ is parameterised with $U_{0}$ and $V$.

- In the risk analysis the variables $\mathbf{X}$ are specified. In the deterministic approach this step would only consist in the determination of the aerodynamic coefficients of the vehicle and the definition of the ideal gust. On the contrary, in the probabilistic approach the statistics of the coefficients and of the ideal gust have to be set. When the parameters $\mathbf{X}$ have been set (2.1) becomes:

$$
p_{F}=p_{F}\left(U_{0}, V\right) \text {. }
$$

This relation can be rearranged as:

$$
U_{0}=U_{0}\left(p_{F}, V\right)
$$

which gives the maximal bearable mean wind velocity $U_{0}$ for a given probability of failure and driving velocity. It can be seen as a generalisation of the conventional 


\subsection{Aerodynamics topics}

CWC and is called in this work probabilistic wind characteristic curve, PCWC:

$$
U_{0}=P C W C\left(p_{F}, V\right) .
$$

The PCWC is the output of the risk analysis. At this point it would be possible to fix, on empirical basis, a limit value $p_{F 0}$ for the probability of failure. In this case $P C W C\left(p_{F 0}, V\right)$ would be a conventional deterministic wind characteristic curve and the crosswind stability task could be carried on in the usual way. On the contrary, in the reliability based approach the PCWC is passed in its general from (2.2) to the risk assessment.

- In the risk assessment the driving velocity and the mean wind velocity are considered in two steps, see Fig. 2.1.

Firstly, the driving velocity $V$ is fixed as scheduled. In the conventional approach the maximal acceptable mean crosswind velocity $U_{0 \max }$ corresponding to the fixed $V$ could now be determined from the CWC. On the contrary, in the probabilistic approach Eq. (2.2) becomes:

$$
p_{F}=p_{F}\left(U_{0}\right)
$$

The mean wind velocity $U_{0}$ is then considered. $U_{0}$ is now a stochastic variable with probability distribution function $f_{U_{0}}$ describing the recurrence of the wind; extreme winds will be discussed in Sec. 2.2.2. The risk $R$ can now be assessed. In the deterministic approach the risk would correspond to the probability that $U_{0}$ overrides $U_{0 \max }$. On the contrary, in the reliability based approach Eq. (2.5) is used. Applying the statistical notation ( $U_{0}$ stochastic variable, $u_{0}$ outcome), the risk is:

$$
R=\int_{0}^{\infty} f_{U_{0}}\left(u_{0}\right) p_{F}\left(u_{0}\right) \mathrm{d} u_{0} \equiv E\left[p_{F}\right] .
$$

The risk can be thus interpreted as the expected value, over the wind velocity $U_{0}$, of the probability of failure $p_{F}$.

By way of example, Fig. 2.2 depicts the risk evaluation for a real case. The vehicle model that will be introduced in Sec. 3 with a driving velocity $V=250 \mathrm{~km} / \mathrm{h}$ and a typical scenario have been considered. In the top subplot two curves are reported: the relation (2.5) evaluated over the range $U_{0}<20 \mathrm{~m} / \mathrm{s}$ using the reliability techniques that will be described in Sec. 4; the assumed exemplary

probability distribution of the wind velocity $f_{U_{0}}$ (Type I extreme, see Table 2.3 on page 35). In the bottom subplot their product, i.e. the integrand of (2.6), is plotted. The resultant risk is $R=0.002$.

\subsection{Aerodynamics topics}

\subsubsection{Railway aerodynamics}

Railway aerodynamics is a general term covering many different phenomena in addition to the crosswind stability: pressure effects (pressure waves in tunnel, pressure gradients 


\section{Crosswind stability}
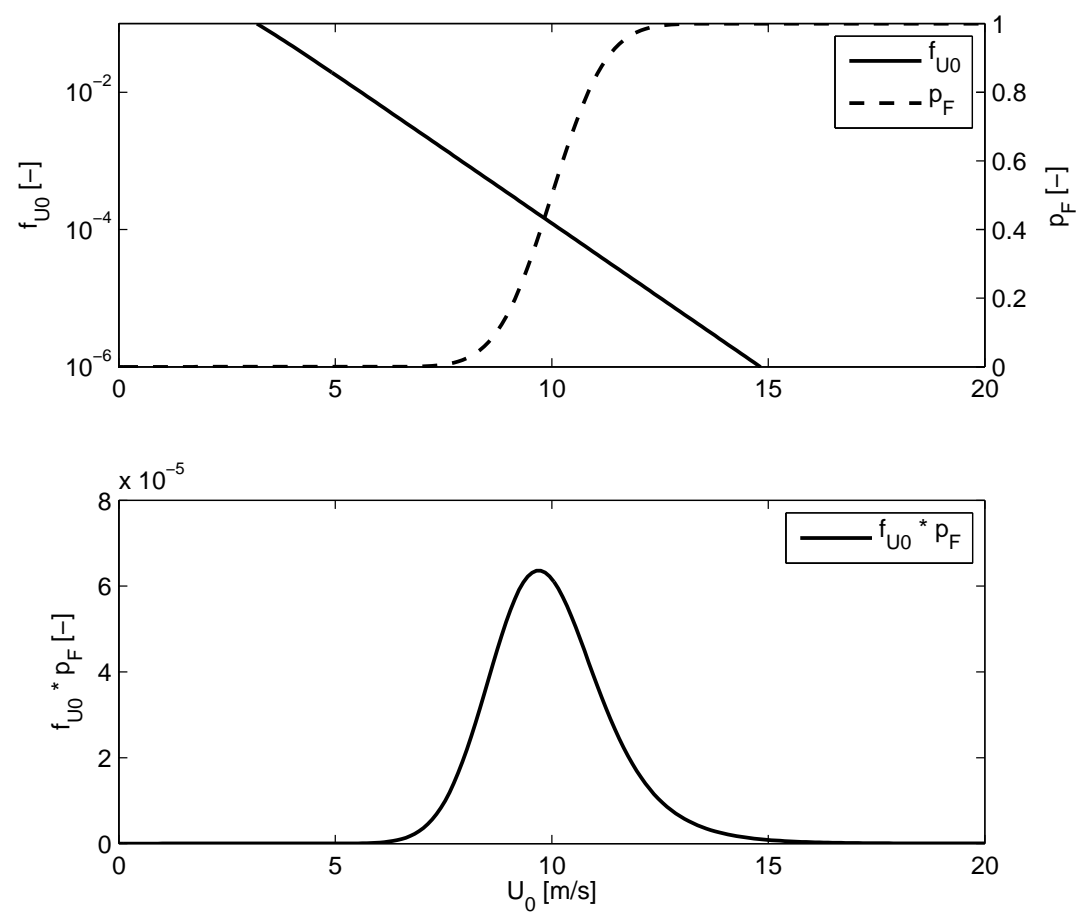

Figure 2.2: Top: PDF of the mean wind velocity $f_{U_{0}}$ (log. ordinate) and probability of failure $p_{F}\left(U_{0}\right)$. Bottom: integrand in (2.6); the risk $R$ is the are spanned by the curve.

during passing manoeuvres, pressure fluctuations in the wake), driving resistance (form drag, viscous drag), etc. Summaries on the topic are given in [Glü85, Soc96, Sch01] and a large overview on ongoing work can be found in [BS03].

The wind angle $\delta$, the yaw angle $\theta$ and the relative wind velocity $U_{r}$ are defined in Fig. 2.3 for a generic case. In this work only pure crosswind, i.e. $\delta=90^{\circ}$, will be considered; it follows that:

$$
\begin{aligned}
U_{r} & =\sqrt{U^{2}+V^{2}}, \\
\theta & =\arctan \left(\frac{U}{V}\right) .
\end{aligned}
$$

The choice of $\delta=90^{\circ}$ does not automatically lead to the worst case but the differences that can be observed using other values of the wind angle are little and can be usually neglected. However, the wind angle can be a relevant factor when the wind direction with respect to the track is taken into account in the risk assessment, see for example [ $\left.\mathrm{I}^{+} 02\right]$.

\section{Bluff bodies}

A basic definition of bluff bodies ${ }^{1}$ is that the flow around such bodies, like most vehicles and buildings, is characterised by large separated regions; on the contrary the flow around

\footnotetext{
${ }^{1}$ German: stumpfe Körper.
} 


\subsection{Aerodynamics topics}

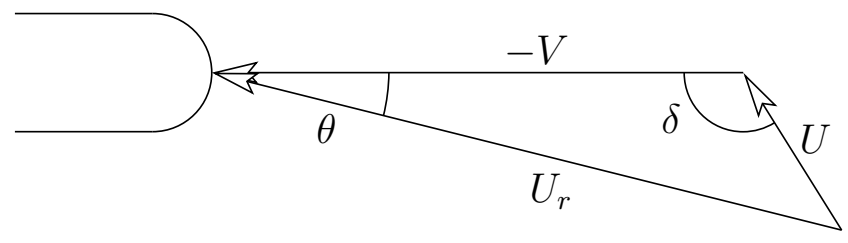

Figure 2.3: Definition of wind angle $\delta$ and yaw angle $\theta$ (top view of the vehicle).

slender bodies ${ }^{2}$, like airfoils, remains attached, [And95]. Moreover, the flow around bluff bodies is often referred to as nonlinear, which briefly means that the nonlinear terms in the equations of motion of the flow cannot be neglected; this is physically due to the fact that the viscous effects influence large regions of the flow.

Contrary to road vehicles, railway vehicles can be seen during normal operation as slender bodies because regions of separated flow are comparatively small and do not have a large influence on the overall flow. However, in the presence of crosswind, railway vehicles must be seen as bluff bodies, because large separated regions with peculiar flow patterns appear on the lee side. The separated, strongly turbulent flow between underbelly and track, which is always present and has e.g. large influence on drag, as well as the wake after the last car of the convoy, are not considered here.

Almost no analytical methods exist for the study or the prediction of the flow around bluff bodies and the few empirical models which are available only suit very simple configurations. Basically only experiments (wind tunnel tests or CFD computations) can provide some information about the flow. Still more complex are the phenomena for time dependent configurations, i.e. when the body moves in a steady flow and/or the flow is unsteady.

The aerodynamic loads acting on a driving vehicle depends in general on both vehicle and wind characteristics and time. Within the scope of vehicle aerodynamics only few quantities are usually considered, [Huc98, Huc02]:

$$
F=F(\underbrace{\text { shape, } A, V}_{\text {body related }}, \underbrace{\delta, \rho, U(z), H(\omega), L_{U}^{x}(z), t}_{\text {wind related }}) .
$$

where $z$ is the height over ground, $A$ a vehicle characteristic area, $V$ the driving velocity, $\delta$ the wind angle, $\rho$ the air density, $U_{0}$ the mean wind velocity, $H$ the aerodynamic admittance, $L_{U}^{x}$ the longitudinal turbulence length, $t$ the time. It must be mentioned that the dependency on $z$ is often neglected, even though the effects of spatial nonhomogeneity, originating mainly from the boundary layer, are known to be influential, see for example [Bak91a].

The description of the aerodynamic coefficients and the questions connected to steady and unsteady phenomena are summarised in the following sections, even though a clear separation between steady and unsteady aerodynamics is not always possible.

\footnotetext{
${ }^{2}$ German: schlanke Körper.
} 


\section{Crosswind stability}

\section{Aerodynamic loads}

The aerodynamic coefficient $C_{F}$ corresponding to a generic aerodynamic load $F$ (side and lift force; roll, pitch and yaw moment) for a given configuration is the value of the load adimensionalised with the dynamic pressure $\frac{1}{2} \rho U_{r}^{2}$ and a characteristic area $A$ of the body. In a planar case, $\theta$ being the yaw angle, Fig. 2.3, the load can be expressed as:

$$
F\left(\theta, U_{r}\right)=\frac{1}{2} \rho A C_{F}(\theta) U_{r}^{2}
$$

The coefficient $C_{F}(\theta)$ can be measured in wind tunnel or computed by CFD by a series of tests or computations for different values of $\theta$. For ground vehicles an almost linear dependence of the coefficients on the yaw angle in the range $\theta= \pm 20^{\circ}$ is observed. This fact is physically well justified by the flow patterns around the vehicles, see for example [Huc98], and is also the reason why the aerodynamic coefficients for $\theta=20^{\circ}$ are often taken as a reference.

In Fig. 2.4 the generic aerodynamic load $F$ is reported over the crosswind velocity for different driving velocities; linearity of the coefficient $\left(C_{F}(\theta)=\bar{C}_{F} \cdot \theta\right.$ with $\bar{C}_{F}=$ const) is assumed. It can be observed that for high driving velocities the load depends almost linearly on the crosswind velocity and only very low yaw angles $\left(<20^{\circ}\right)$ are reached. These facts suggests that a linearisation of the loads would be a good approximation.

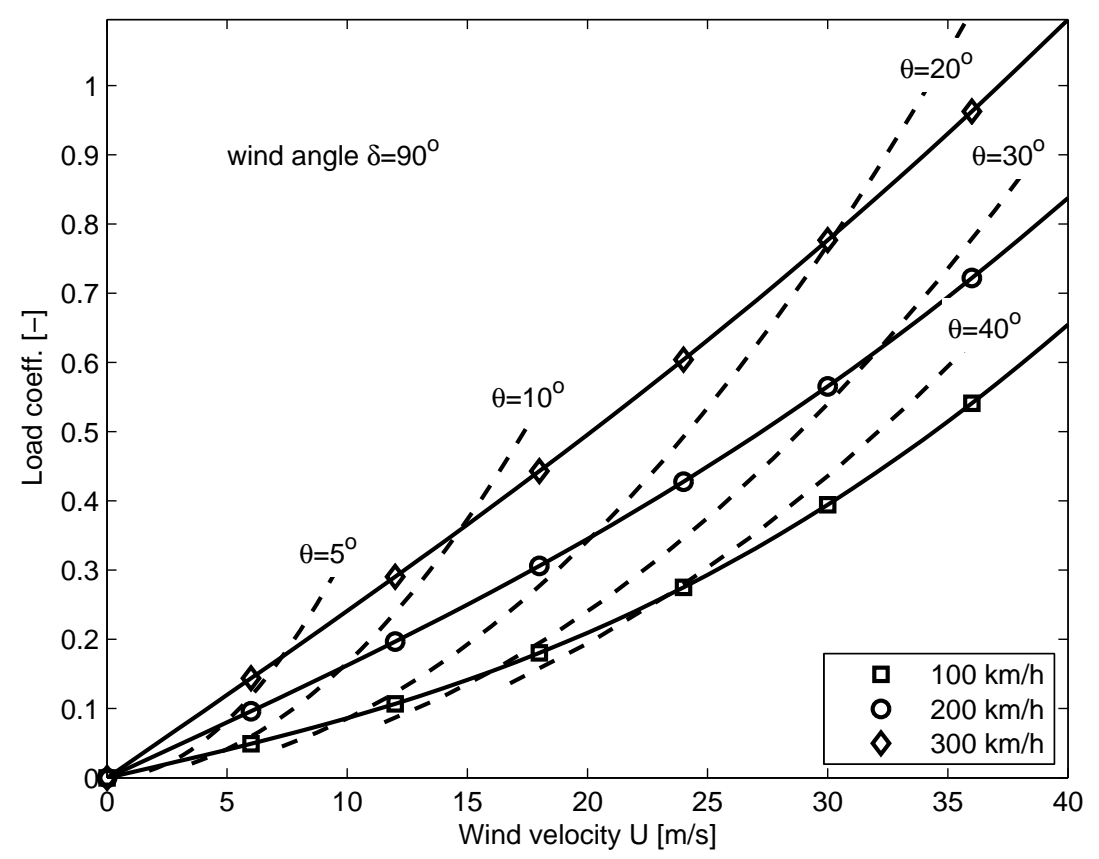

Figure 2.4: Generic adimensionalised aerodynamic load for different driving velocities according to (2.9), having assumed linear aerodynamic coefficient and pure crosswind. The resultant yaw angle $\theta$ is also indicated.

In the quasi-steady approach, Eq. (2.9) is assumed to hold also for time varying $U_{r}$ 


\subsection{Aerodynamics topics}

and $\theta$ :

$$
F\left(\theta(t), U_{r}(t)\right)=\frac{1}{2} \rho A C_{F}(\theta(t)) U_{r}(t)^{2} .
$$

The underlying physical assumption is that the flow at every instant $\bar{t}$ is equivalent to a steady flow for the fixed configuration $\bar{\theta}=\theta(\bar{t})$ and $\bar{U}_{r}=U_{r}(\bar{t})$.

In general, a slight enhancement of (2.10) can be achieved introducing the dependency to $\dot{\theta}$ in the style of aeroelasticity, [BAH96]. The aerodynamic force is then (time dependency omitted):

$$
F\left(\theta, \dot{\theta}, U_{r}\right)=\frac{1}{2} \rho A C_{F}(\theta, \dot{\theta}) U_{r}^{2}
$$

In this approach a harmonic motion with frequency $\omega$ is assumed and a linearisation of the coefficients is usually performed:

$$
C_{F}(\theta, \dot{\theta}, \omega)=c_{\theta}(\omega) \theta+c_{\dot{\theta}}(\omega) \dot{\theta} .
$$

The aerodynamic derivatives $c_{\theta}$ and $c_{\dot{\theta}}$ have been often measured experimentally for road vehicles, see for example [MP04], and were used for stability studies on cars, e.g. [CDMZ00].

The aeroelastic approach is beneficial when the dynamic response to the aerodynamic loads of a vehicle having some degree of freedom with respect to the flow is examined. This is not the case of the gust response problem related to the crosswind stability task, where the dependency of $\theta$ on time is not due to a motion of the vehicle but to a change in the crosswind velocity. The dependency of the coefficients on $\dot{\theta}$ is thus not relevant. However, the dependency of the coefficients on the frequency $\omega$ of the hypothetical harmonic motion is remarkable because it represents the most basic model for unsteady aerodynamics. This dependency, addressed with the term aerodynamic admittance, is discussed below where unsteady aerodynamic effects are considered.

\section{Steady aerodynamics}

Steady aerodynamics occurs when the body is fixed with respect to a steady flow. Every body's translatoric motion with constant velocity can be reduced to the case of a fixed body by taking the relative incident flow as incoming flow; this is the case of a driving vehicle, where the vehicle moves with respect to the crosswind.

The steady aerodynamics of ground vehicles is complicated by phenomena that do not occur at all in aeronautical applications and only partially in building aerodynamics. The most relevant ones are the relative motion between ground and vehicle, the turbulent content of the wind and the ground boundary layer, [Huc98]. For railway and road vehicles these phenomena have been systematically studied only in absence of crosswind. This is mainly due to the difficulties in quantifying such effects in the presence of crosswind. The main consequence is that large uncertainties affect the available information.

Furthermore large fluctuations in the loads can actually arise in steady flow and are mainly due to two phenomena, [WH04]: 


\section{Crosswind stability}

- Turbulence. Fluctuations induced by the small scale turbulence content of the wind are well known and could be observed in wind tunnel tests equipped with turbulence generators but also in full scale tests. However, partly contradictory results were produced and definitive statements on this topic are still missing.

- Vortex shedding. This well known phenomenon induces a periodic modification of the flow pattern around the body and and thus fluctuations of the pressure distribution. Recently it was studied also for vehicles in crosswinds by wind tunnel tests and CFD computations but reliable information is lacking.

The fluctuations are quantified by the peak/mean ratio. A typical trend observed by different authors is that the ratio is $>1$ for low yaw angles, which are the most interesting for the crosswind stability, and $<1$ for large ones. A further discussion and references will be given in Sec. 3.1.2.

\section{Unsteady aerodynamics}

It has already been noted that (2.9) still holds for "slowly" time varying $U_{r}(t)$ and $\theta(t)$ but the validity of such approximation, i.e. the limit separating steady from unsteady aerodynamics, is not easy to determine; a possible criterion is for example the reduced frequency, [BAH96].

Many approaches have been developed in aeronautics to model unsteady aerodynamic effects on wings; however, the assumptions on which they are based do not allow their use for bluff bodies, because of the intrinsic complexity of the separated flow. The investigations on unsteady aerodynamic effects around bluff bodies trace back almost exclusively to the field of building aerodynamics, especially suspended bridges, [Sca97]. Some attempts to establish semianalytical models for ground vehicles have been done, e.g. [HE73, Fil03], but the only approach which is available in practice is the experimental aerodynamic admittance, [Bak91b]. With regard to ground vehicles, this term is used in the literature to designate two different cases. Even though they refer to the same physical phenomenon and the definition of the admittance is the same, see below, the cases can be regarded as distinct:

- Turbulence. The higher the frequency of the velocity fluctuations measured on a location in the oncoming flow, the more little are their effects on the aerodynamic loads, because the fluctuations are due to very small eddies which can influence the flow only locally. On the contrary, low frequency fluctuations correspond to large eddies with dimensions are comparable with those of the vehicle and capable to influence the overall flow and thus the aerodynamic loads. This admittance is connected with the spatial properties of the flow and was originally introduced in building aerodynamics. Measured admittances for railway vehicles are reported in [Coo81, CCDT03, B ${ }^{+}$04].

- Transient flow. To a nonturbulent flow varying in intensity or direction with a fixed frequency correspond oscillations in the aerodynamic loads with a specific amplitude and phase delay. This can be experimentally studied by motion of the 


\subsection{Aerodynamics topics}

vehicle in a steady flow or by gust generators. Special transient flow patterns around the vehicle can arise and/or the flow patterns typical of the steady flow regime cannot fully develop. This admittance is connected with the temporal variation of the flow, and was firstly defined in aeroelasticity. Measured admittances can be found in [HE83, PRI01, MP04]. Some authors refer to this admittance as magnification.

The definition of admittance for the generic aerodynamic load $F$ is:

$$
\left|H_{F}(n)\right|^{2}=\frac{S_{F}(n) U_{0}^{2}}{4 S_{U}(n) F_{0}^{2}},
$$

where $S_{F}, S_{U}$ are the power spectral densities and $F_{0}, U_{0}$ the mean values of the load $F$ and the wind speed $U$. This expression derives from the turbulence approach, which leads to the factor 4 in the denominator, see e.g. [SS96]. In the transient flow case the factor 4 should be omitted, as some authors do, and the admittance is just the transfer function between the non-dimensional wind speed and the non-dimensional load.

In Fig. 2.5 the side force admittance acquired by transient flow tests reported in [HE83] is plotted. It will be used in Sec. 3.2.2 to assess the unsteady aerodynamic loads deriving from different gust shapes.

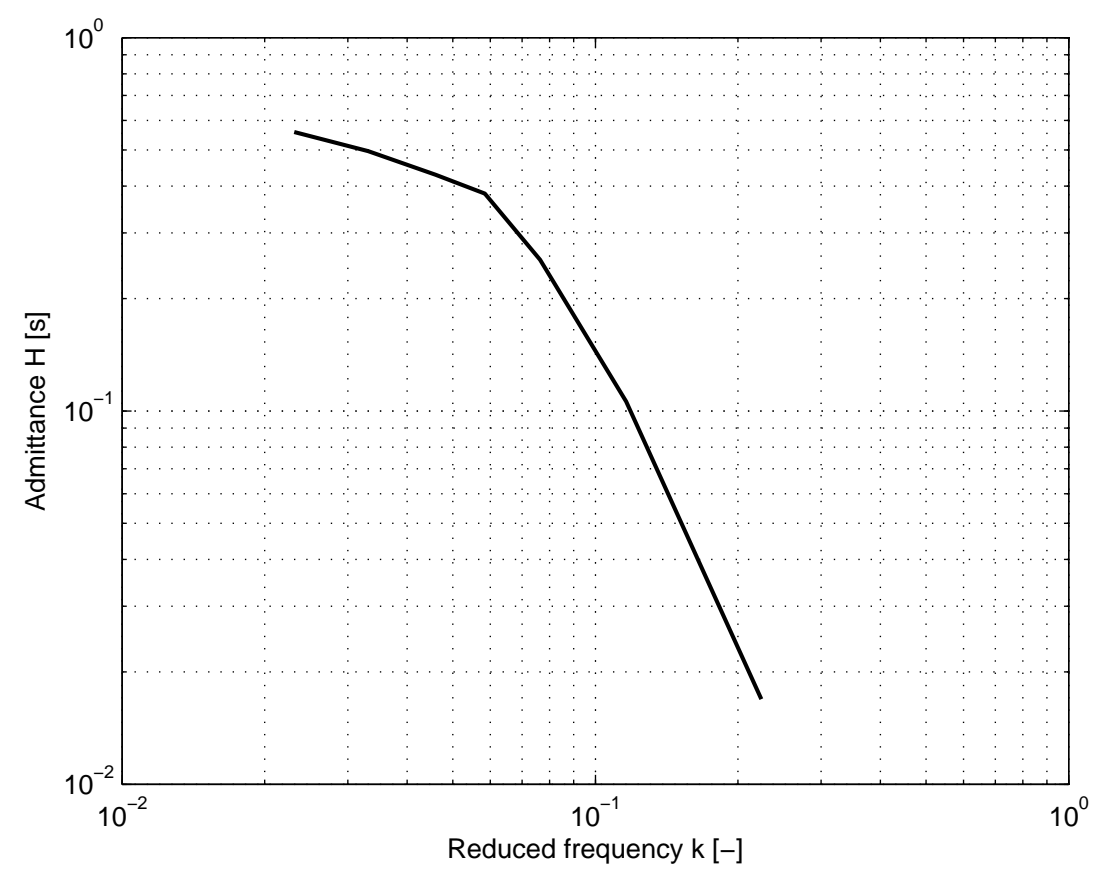

Figure 2.5: Side force admittance reported in [HE83].

\section{Nonhomogeneous flow}

When a vehicle drives through a gust of limited spatial extension different sections of the vehicle are exposed at any time to different wind velocities. This fact is of large 


\section{Crosswind stability}

importance for road vehicles because it contributes to the overshoot in the yaw moment, which is a very sensitive parameter in the driver-car control loop ${ }^{3}$, [Huc98]. For railway vehicles the effects of nonhomogeneous flow around the bow are supposed to be of some importance because the bow region is responsible for a large part of the overall lift. In the past the problem was extensively studied with moving model tests in ship model basin, see for example [NS76], where a sharp edged gust can be reproduced with relatively litlle effort and high accuracy. In [Lip99] a modified ideal gust profile was proposed to account for nonhomogeneous flow (crosswind at tunnel exit) but it is not congruent with experimental results.

As in this work a spatially infinite ideal gust will be used, see Sec. 2.2.2, the problem of nonhomogeneous flow can be neglected. Moreover, in the last years the attention on sharp edged gusts is decreased, because more realistic smooth gust models were developed; for such shapes the unsteadiness is supposed to be more important than the nonhomogeneity.

\subsubsection{Wind}

\section{Steady wind}

When the fluctuations of the wind velocity are low with respect to the mean value, the wind is said to be steady. According to this abstraction the wind velocity is equal to its mean value and thus constant. However, many phenomena which significantly influence the aerodynamic loads have still to be considered. The most important are:

- Turbulence. Steady wind has a turbulence content which is described by statistical indicators (turbulence intensity, turbulence length scale, etc.). Moreover, turbulence can be locally strongly influenced by the interaction of the wind with infrastructures along the track. Some authors, see for example [Huc02, SS96], tried to summarise the effects of turbulence on the flow around bluff bodies. One of the general results is that the effects on the flow topology, and thus finally on the aerodynamic loads, are particularly large for bodies on which the location of the flow separation is not fixed by the body shape (e.g. sharp edges) but results from the natural detachment of the boundary layer. This surely applies to modern railway vehicles (see for example the very rounded bow of the German ICE3).

- Velocity profile. The wind velocity is a function of the height over ground. This dependency is due to the ground boundary layer, to the configuration of the ground etc. and largely influences the aerodynamic loads. The velocity profile becomes a critical issue in some typical railway scenarios: on embankments, for example, significantly modified velocity profiles are observed additionally to higher mean wind velocities. Similarly, high mean wind velocities are to be expected on bridges, because of the height over ground (i.e. outside the ground boundary layer); on the

\footnotetext{
${ }^{3}$ However, the yaw moment overshoot is observed also in homogeneous flow, i.e. when the whole vehicle is exposed at any time to the same wind velocity.
} 


\subsection{Aerodynamics topics}

other hand, the bridge itself influences significantly the flow, leading to local speedups and peculiar velocity profiles around the bridge section.

Only few of the effects noted above can be described deterministically (e.g. bridge or embankment passing) and their effects quantified. Most of them can be measured in practice only with very large effort (e.g. turbulence intensity, wind profile) and their effects on the aerodynamic loads are not known or too difficult to consider.

An additional problem is given by the relative motion between vehicle and wind, i.e. how the turbulence intensity and the other indicators are experienced by a moving observer, [Coo84, WSH95]. In general a vehicle driving in a crosswind is exposed to a lower turbulence intensity because the incident flow is composed of the relative flow due to the vehicle motion, which is perfectly smooth, and to a minor extent by the turbulent natural wind.

\section{Unsteady wind}

When the unsteadiness of the wind is large the fluctuations of the wind velocity cannot be neglected or reduced to indicators. From a physical point of view, the frozen turbulence hypothesis is widely used. According to this simplification the wind velocity fluctuations are advected with the mean velocity. In measurement technology it is also referred to as Taylor's hypothesis and allows time series measured at a single location to be interpreted as spatial variations. It only holds if the relative turbulence intensity is low; that is, $u / U_{0} \ll 1$ where $U_{0}$ is the mean velocity and $u$ the fluctuating velocity. Then the substitution $t=x / U_{0}$ is a good approximation, $x$ being the alongwind coordinate.

Unsteady wind can be considered with good approximation to be a gaussian stochastic process and is thus completely characterized by the power spectral density. Many formula have been proposed for this quantity but the most common one is due to Von Karman and is also used in the TSI norm:

$$
S_{U}(n)=\frac{4 \sigma_{U}^{2} \frac{n L_{U}^{x}}{U_{0}}}{\left[1+70.7\left(\frac{n L_{U}^{x}}{U_{0}}\right)^{2}\right]^{5.6}}
$$

where $n$ is the frequency and $L_{U}^{x}$ is the integral length scale of the wind speed $U$ in the longitudinal direction $x$ (i.e. parallel to $U$ ), [SS96]. The spectrum is plotted in Fig. 2.6 for the frequency range of interest as defined in the TSI. A realisation of the process with a corresponding exemplary wheel unloading computed with the multibody model that will be introduced in Sec. 3 is reported in Fig. 2.7; wheel unloading will be formally defined in Sec. 2.3.

Unsteady wind must be modelled according to the nature of the body with which it interacts. In Sec. 2.2.1 it was discussed that railway vehicles are bluff bodies, for which three approaches are usually used, [Rus82, Soc84]:

- Gust factor approach. A constant wind velocity equal to the peak value is assumed; the problem is thus reduced to a steady case. This approach is used when the 


\section{Crosswind stability}

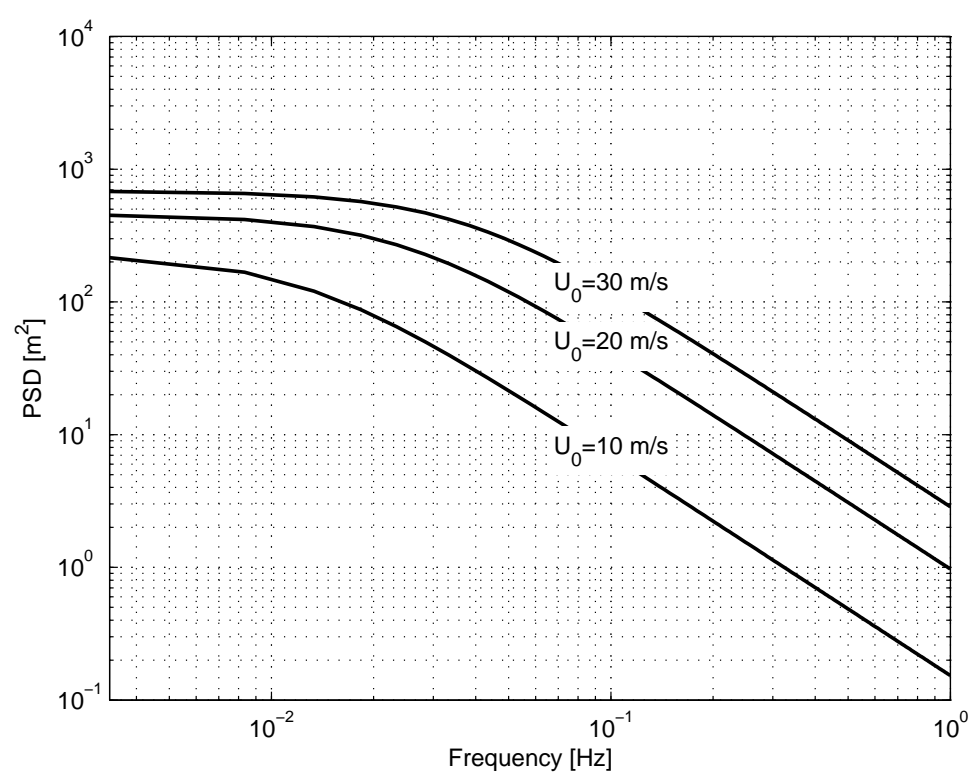

Figure 2.6: Von Karman wind spectrum for different mean wind velocities in the frequency range fixed in the TSI.

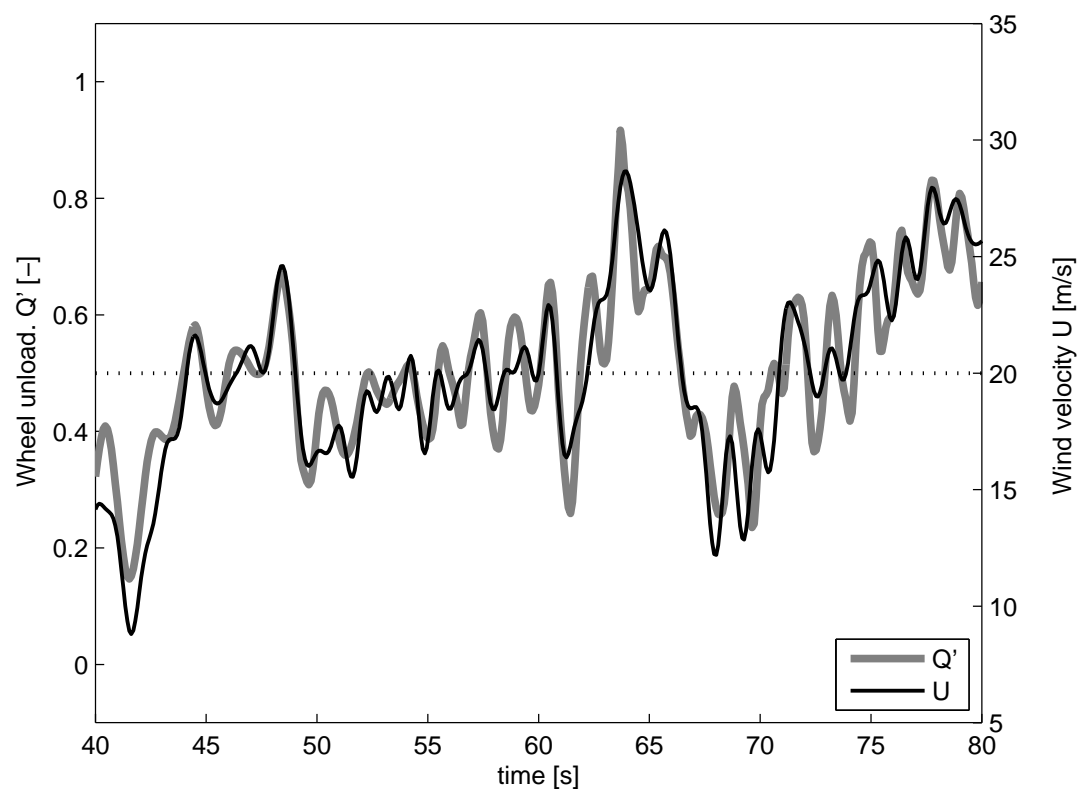

Figure 2.7: Wind sample from the spectrum of Fig. $2.6\left(U_{0}=20 \mathrm{~m} / \mathrm{s}\right)$ with exemplary response of the vehicle (unloading of the leading wheel on the wind side).

dynamic response of the body is not of interest, like buildings without relevant dynamics. 


\subsection{Aerodynamics topics}

- Frequency domain approach. The wind is described by its power density spectrum and the analysis is performed in the frequency domain. It is used when the interaction between dynamic load and dynamic response of the body is relevant, e.g. some kind of buildings (bridges, towers), wind power plants.

- Ideal gust approach. A time-dependent deterministic wind time history is assumed and the system response is usually computed by time integration. This approach is used when the maximal dynamic response of the body has to be checked, e.g. vehicles, wind power plants.

Each of the approaches can be and has been used in railway aerodynamics for the crosswind stability task. The gust factor approach is still used in the regulations of some countries. The frequency domain approach has been used to check that the natural frequencies of the car motion cannot be excited by the wind, [Bak91c]. The ideal gust approach is currently widely diffused and used, for example, in the TSI norm.

Finally, constrained simulation, [BC02], must also be mentioned. It is a relatively new approach, originally developed for the analysis of the maximal response of wind power plants. It allows the use of wind time histories that are realisations of the stochastic process but have given characteristics, e.g. a given peak value. In this way the advantages of the ideal gust approach are combined with a correct description of the wind. This methodology has not been applied yet to the crosswind stability task.

\section{Ideal gusts}

An ideal gust is a variation of the wind velocity defined by a simple, usually analytical, function of time. Such variation occurs in the same direction of (or "following") the main wind speed (longitudinal gust). According to the frozen turbulence hypothesis a gust does not evolve and is transported with the mean wind velocity $U_{0}$ which is assumed constant. In this work only pure crosswind, i.e. wind perpendicular to the track $\left(\delta=90^{\circ}\right)$, is considered; the gust is thus a pure crosswind gust.

In Fig. 2.8 a) an ideal crosswind gust is depicted in two different moments, $t^{\prime}$ and $t^{\prime \prime}=t^{\prime}+\Delta t$; during $\Delta t$ the gust moves of a distance $U_{0} \cdot \Delta t$. At the position $s_{0}$ on the track the crosswind velocity at $t^{\prime}$ and $t^{\prime \prime}$ is respectively $U^{\prime}$ and $U^{\prime \prime}$. As the mean wind velocity $U_{0}$ is constant, the spatial coordinate across the track, $y$, can also be seen as time coordinate, $t$. This is to say, the extension of the gust along $y$ is also the time history of the crosswind velocity as measured by a fixed observer at $s_{0}$.

The spatial extension of the gust, Fig. 2.8 b') and b"), has now to be considered.

Fig. 2.8 b') Real gusts are limited in space and their extension is quantified by the spatial correlation function, [SS96]. In Fig. 2.8 b') the ideal extension of the gust along the track at the moments $t^{\prime}$ and $t^{\prime \prime}$, i.e. the crosswind velocity $U$ as a function of the track coordinate $s$, is reported. It is assumed for simplicity's sake that the observing location $s_{0}$ is also the location where the gust reaches its maximum value. The field of the crosswind velocity can be imagined as a moving hill.

The model of Fig. 2.8 b') is very close to reality and is used in the TSI. However, it has many drawbacks: 


\section{Crosswind stability}

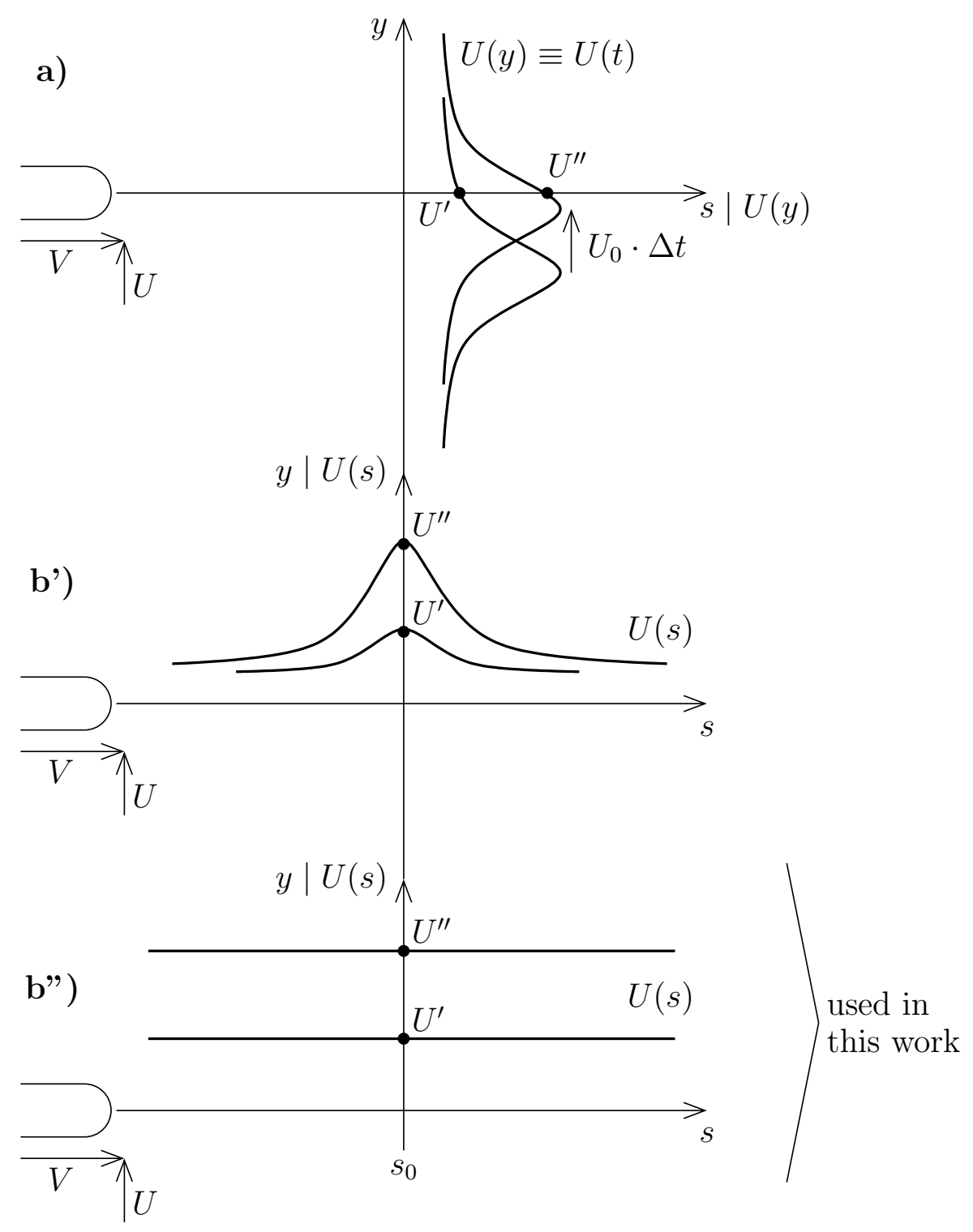

Figure 2.8: Crosswind gust, two moments $\left(t^{\prime}\right.$ and $\left.t^{\prime \prime}=t^{\prime}+\Delta t\right)$ considered. $s$ is the coordinate along the track; $y$ is the coordinate across the track and also the wind direction. a) Crosswind velocity distribution across the track at location $s_{0}$. b') Crosswind velocity distribution along the track, considering spatial correlation. b") Crosswind velocity distribution along the track, neglecting spatial correlation, as used in this work. 


\subsection{Aerodynamics topics}

- The model is complex and the knowledge of the spatial correlation is necessary.

- The crosswind seen from the moving train is the resultant of the time history of the gust with respect to a fixed observer, its spatial extension and the motion of the vehicle. This means that the resultant crosswind history to be used in the simulation is defined by a large number of parameters.

- Because the train drives through the gust, the resultant crosswind time history seen by the train is dependent on the driving velocity, making a comparison of the results for different driving velocities difficult.

- When the train drives into the gust, complex unsteady aerodynamic phenomena arise because the flow seen by the train is not only varying in time but is also nonhomogeneous in space. An appropriate aerodynamic model should be thus be created.

Fig. 2.8 b") A simplified approach is reported in Fig. 2.8 b"). In this case the gust is supposed to be infinitely extended, i.e. infinitely correlated, and the motion of the vehicle along the track has no influence at all. The field of the crosswind velocity can be imagined as an unlimited wave moving across the track.

This approach, widely used in practice, is physically not very close to reality. On the other hand, a substantial simplification of the model is obtained and the drawbacks arising when considering the spatial correlation are avoided. In practice the information which is needed to define the model can be directly derived from the data measured by a single anemometer at a fixed location on the track.

It must be stressed that the time histories of the crosswind seen by the moving vehicle in the approaches of Fig. 2.8 b') and b") are substantially different. In the former the most influence comes from the spatial extension of the gust through which the vehicle drives; in the latter the time history is only due to the time variation of the crosswind intensity and is thus the same for the moving vehicle and for a fixed observer.

\section{Extreme winds}

Extreme winds are described by the probability distribution function of the peak velocity over a fixed period, usually one year, [SS96]. The so called Type-I extreme distribution is usually used, see Sec. 2.4.2, but the choice of the distribution that best fits the wind data is still an open question. Even though alternative statistical techniques to manage extremal data are being developed, e.g. [PK04], asymptotic distributions are still widely used in practice, for example in the German standards for wind loads on buildings, [DIN05].

As discussed in Sec. 2.1.3, the distribution of the extreme winds, i.e. the distribution of $U_{0}$, is a matter of the risk assessment and not of the risk analysis. Moreover, the fact that the gust parameters (gust amplitude and gust length) depend on the mean wind speed $U_{0}$ was neglected in this work. 


\section{Crosswind stability}

It must be observed that the maximum wind velocity which can be encountered in practice by a railway vehicle during operations is limited by many factors. In practice strong winds can lead to an interruption of the operations because of the presence of debris on the track or the damage of catenary and catenary support. The inevitable interruption of the operations avoids that the train is exposed to such extreme winds.

It is interesting to note that, according to the Beaufort-Scale, tree branches are expected to break at wind speeds of $17 \div 20 \mathrm{~m} / \mathrm{s}$ (grade 8 ). Not too ironically this fact has been seen as a "natural" warning system for the train driver in countries (like Germany) that do not have anemometers mounted along the tracks, [OSB02]. Uprooted trees, which can definitely destroy the catenary or, even worse, lay on the track, are expected at grade 10 , i.e. already at $24 \div 28 \mathrm{~m} / \mathrm{s}$.

\subsubsection{Analysis tools}

In the previous sections it was discussed that the main concern of aerodynamics is to establish models of the wind and the vehicle. Techniques to establish wind models (e.g. ideal gust) from measured wind data are not addressed here; the reader is referred to the vast literature on wind engineering, e.g. [SS96]. On the contrary, the tools for the assessment of an aerodynamic vehicle model (aerodynamic coefficients and possibly the admittance function) are briefly reviewed.

\section{Experimental techniques}

The aerodynamic coefficients are usually evaluated by static wind tunnel tests. However, even considering only steady phenomena, many systematic errors cannot be avoided, see for example [BH96]. The most important are:

- Ground motion. In reality the ground moves relatively to the vehicle, whereas in the tests ground and vehicle are fixed. Ground motion can only be simulated for $0^{\circ}$ yaw, e.g. by moving belts substituting the ground, but is technically not possible in the presence of crosswind.

- Ground flow. In reality the angle between vehicle and flow is the yaw angle whereas the angle between ground and flow is the wind angle, because the ground does not move; on the contrary, in the tests with fixed ground both angles are equal to the yaw angle. This is particularly critical when embankment effects are considered.

- Scaling. Small models have to be used in practice to reach acceptable Reynolds numbers. The consequence is that details, especially in the underbelly regions, are very poorly reproduced.

- Turbulence. Turbulence conditions (intensity and length scale) equivalent to those experienced by the vehicles driving in crosswinds are difficult to reproduce.

- Boundary layer. A velocity profile equivalent to the real one cannot be easily created; further on, it cannot be correct at the same time for the vehicle and the ground, see above. 


\subsection{Driving dynamics topics}

Still more difficult is the simulation of transient effects like gusts. A solution, which also helps against some of the problems described above, are moving model tests, see for example $\left[\mathrm{B}^{+} 03\right]$. On the other hand, many drawbacks arise: very small models have to be used, force measurements are affected by noise, etc. Other solutions, like gust generators in conventional wind tunnels, [DR99], suffer from many limitations and are seldom used.

Finally, full scale tests in real environment are attractive because no models are needed but are expensive and not very efficient, [MH02]. This is due to the fact that the aerodynamic loads are computed indirectly from the measured wheel forces using a multibody model of the vehicle. The results are very scattered because of the superposed effects of track irregularities and real wind; moreover, large errors are likely to arise from unsteady aerodynamics effects, which are usually not considered in the computational model. An alternative is given by full scale tests with staying vehicle, [B ${ }^{+}$04]; however, the validity of the result for the moving vehicle is not guaranteed.

\section{Numerical techniques}

Computational Fluid Dynamics is a large family of techniques that allow the simulation of the flow around a body and thus the computation of the resulting aerodynamic loads, [And95]. The main advantage is the possibility to study virtually any configuration but is counterbalanced by many drawbacks, the most relevant being the poor accuracy. This is mainly due to the simplifications introduced by the empirical turbulence models, as a numerical solution of the complete equations for turbulent flow cannot be afforded in practice. Furthermore an estimation of the error is not possible at the moment, [Kar02, AIA98].

It must be stressed that the computation of the flow around a driving vehicle is a very challenging task because of the large separated regions, the high Reynolds number, and the effects of turbulence. Even neglecting the flow in the underbelly region, which is extremely complex because of the presence of bogies etc., satisfying results are still missing and work is in progress, [Die03].

\subsection{Driving dynamics topics}

Large literature exists on the driving dynamics of railway vehicles [Kru82, DA88, KS03, Wic03]. In this section only few specific items directly connected to the crosswind stability task are addressed.

\subsubsection{Definitions}

\section{Wheel forces}

The Wheel/Rail contacts are the core of a railway system, being responsible for the support and the guidance of the vehicle. The contact forces are due to form and force 


\section{Crosswind stability}

closure and have also to react to aerodynamic excitations which are the only significant external load acting on a vehicle during normal operation.

Of the complex kinematic and dynamic phenomena in the $\mathrm{W} / \mathrm{R}$ contact only the lateral and vertical resultant forces are relevant for the crosswind stability task; they are respectively denoted as $Y$ and $Q$. Such forces are schematically displayed in Fig. 2.9 for a right wheel, assuming for simplicity a one-point contact.

Given a vertical force $Q$, also noted as wheel load, the adimensional quantity wheel unloading can be introduced:

$$
Q^{\prime}=1-\frac{Q}{Q_{0}}
$$

$Q_{0}$ being the static wheel force, i.e. the vertical force of the still standing vehicle on horizontal track.

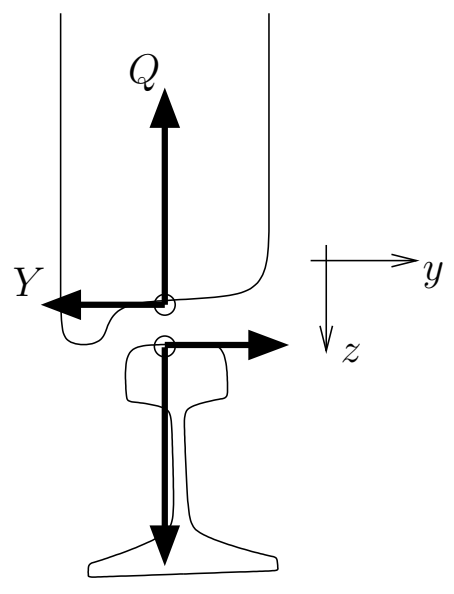

Figure 2.9: Wheel forces.

\section{Criteria and indicators for crosswind stability}

Criteria Almost all the riding safety criteria were used to be considered when dealing with aerodynamic loads, [Sac83]: derailment, track shift, clearance, lateral displacement of pantograph, car lateral acceleration, etc. In more recent times the interest focused on track shift, derailment (flange climbing) and overturning, which are for example specified in the RIL401. At the present time the criterion which is mainly or exclusively taken into account is overturning.

It must be mentioned that the track shift criterion, for which the Prud'homme formula is typically used, can be more restrictive than the overturning criterion, [Lip99]. On the other hand, track shift is mainly a problem for the track operator but it does not directly affect the driving safety, as long as the shift is not so large that it can endanger successive vehicles. Furthermore, the problem of track shift is much more serious for the case of tilting vehicles, which systematically produce high horizontal forces in the curve, than for the case of strong crosswind, which is a very rare event.

Indicators The choice of an appropriate indicator for the overturning criterion is not subject of this work and the conventional wheel unloading indicator is used and applied to the leading wheel on the wind side. It must be noted that the wheel unloading indicator has been proved to be very restrictive and to predict overturning inefficiently. Moreover, it stops working when complete unloading is reached $\left(Q^{\prime}=1\right)$, independently from the severity of the wheel lift. These facts are well documented in [Lip99]. Common alternative indicators are the intercept method and the moment method. The former quantifies the overturning risk referring to the acting point of the overall resultant vertical $\mathrm{W} / \mathrm{R}$ force for a wheelset, a bogie or a whole car; the main advantage is that the $\mathrm{W} / \mathrm{R}$ forces on both wind and leeward side are considered. On the contrary, the moment 


\subsection{Driving dynamics topics}

method is based on the steady equilibrium around the leeward rail of the forces acting on the vehicle; this method is not based only on $\mathrm{W} / \mathrm{R}$ forces but is difficult to apply because all forces and their acting point must be estimated.

Finally, it must be pointed out that even though the choice of the indicator can dramatically influence the results of the crosswind stability analysis the reliability based approach proposed in this work is completely independent from such choice.

\subsubsection{Effects of aerodynamic loads on driving dynamics}

The effects of aerodynamic loads are now briefly addressed referring to the specific vehicle used as a reference in this work. Nonetheless, the results are qualitatively general and well represent the behaviour of a generic vehicle. The computational models will be described in Sec. 3.

\section{Steady phenomena}

When only steady phenomena are considered the crosswind stability is reduced to a static problem. In this case the $\mathrm{W} / \mathrm{R}$ contact dynamics does not play any role and only the lateral displacement of the wheelset compatibly with rail and track gauge must be taken into account. The contributions of the different loads to the unloading (computed with the linear vehicle model of Sec. 3.1.1) are reported in Fig. 2.10, left side. It can be observed that the effect of the roll moment is largely predominant and that the shares are almost constant over the velocity range. The fact that the pitch moment $M_{y}$ increases the unloading for low wind velocities is due to the characteristics of the corresponding coefficient, see Fig. 3.3 on page 43.

It can be observed that crosswind does not only influence the crosswind stability but also the overall driving resistance:

- The aerodynamic resistance is increased because the relative wind velocity $U_{r}$ and the drag coefficient are larger in the presence of crosswind in still air.

- Crosswind also induced a rolling resistance because of the nature of the wheel rail contact. The lateral load on the car can be carried by the $\mathrm{W} / \mathrm{R}$ contact in two ways: by cross slip or by displacement of the contact point towards the flange, significantly increasing the drill slip. In both cases friction processes require some energy and thus additional rolling resistance originates.

\section{Unsteady phenomena}

When unsteady aerodynamic loads (gusts) are considered, the dynamical behaviour of the vehicle must be taken into account. In Fig. 2.10, right side, the contributions of the loads to the peak unloading is reported for a typical gust scenario, showing a similar behaviour as in the steady case. However, a better insight in the unsteady phenomena is given by the spectral power density of the response.

In Fig. 2.11 the exemplary adimensionalised spectra of the responses to a step gust (computed by the complete multibody model, see Sec. 3.1.1) are reported. The wheel 


\section{Crosswind stability}
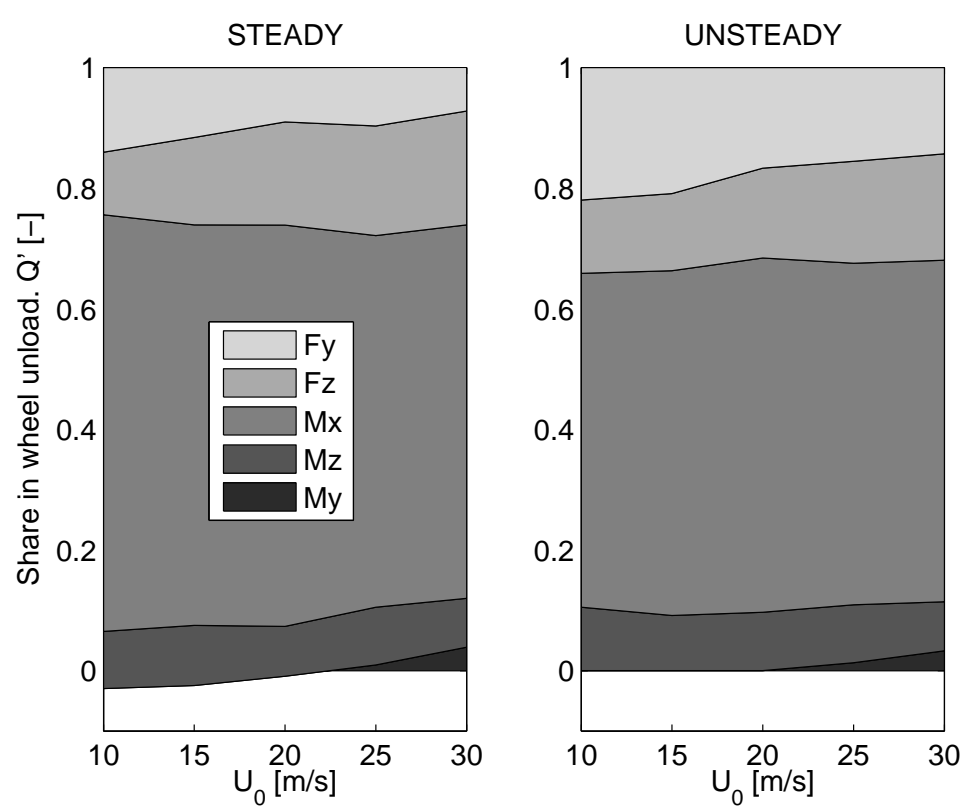

Figure 2.10: Contributions of the aerodynamic loads to the wheel unloading, computed with the linear vehicle model: steady response (left) and peak response to typical gust scenario (right).

unloading spectrum has a major peak around $0.7 \mathrm{~Hz}$ (top subplot, continuous line) that corresponds to the car sway motion as confirmed by the roll angle $\phi$ (bottom subplot, continuous line). Further peaks of the wheel unloading spectrum are at $1.2 \mathrm{~Hz}$ and 1.7 $\mathrm{Hz}$ and can be traced back to the vertical motion of car and bogie (bottom subplot, dashed and dot-dashed lines). This fact is supported by the spectrum of the wheelset unloading (top subplot, dashed line), where the peaks at $1.2 \mathrm{~Hz}$ and $1.7 \mathrm{~Hz}$ are evident. As to be expected, the wheelset unloading spectrum has no peak at $0.7 \mathrm{~Hz}$, because the roll motion influences the load distribution between the wheel but not the global load on the wheelset.

In Table 2.2 the frequencies observed in the spectra of the nonlinear response are compared with the eigenfrequencies computed by modal analysis with the same model. As the eigenfrequencies are computed on a system linearised around the nominal position, the values are slightly different but the distribution of the eigenmodes confirms the considerations presented above, up to the bogie vertical motion that cannot be directly recognised. The car pitch angle $\gamma$, predominant in the fourth eigenmode, was not reported in Fig. 2.11 for the sake of clarity.

\subsubsection{Analysis tools}

Similarly to the case of vehicle aerodynamics, both experimental and computational techniques exist for the analysis of the driving dynamics. The former comprehend mainly full scale and roller rig tests; the latter cover a large spectrum of analytical and numerical 


\subsection{Driving dynamics topics}
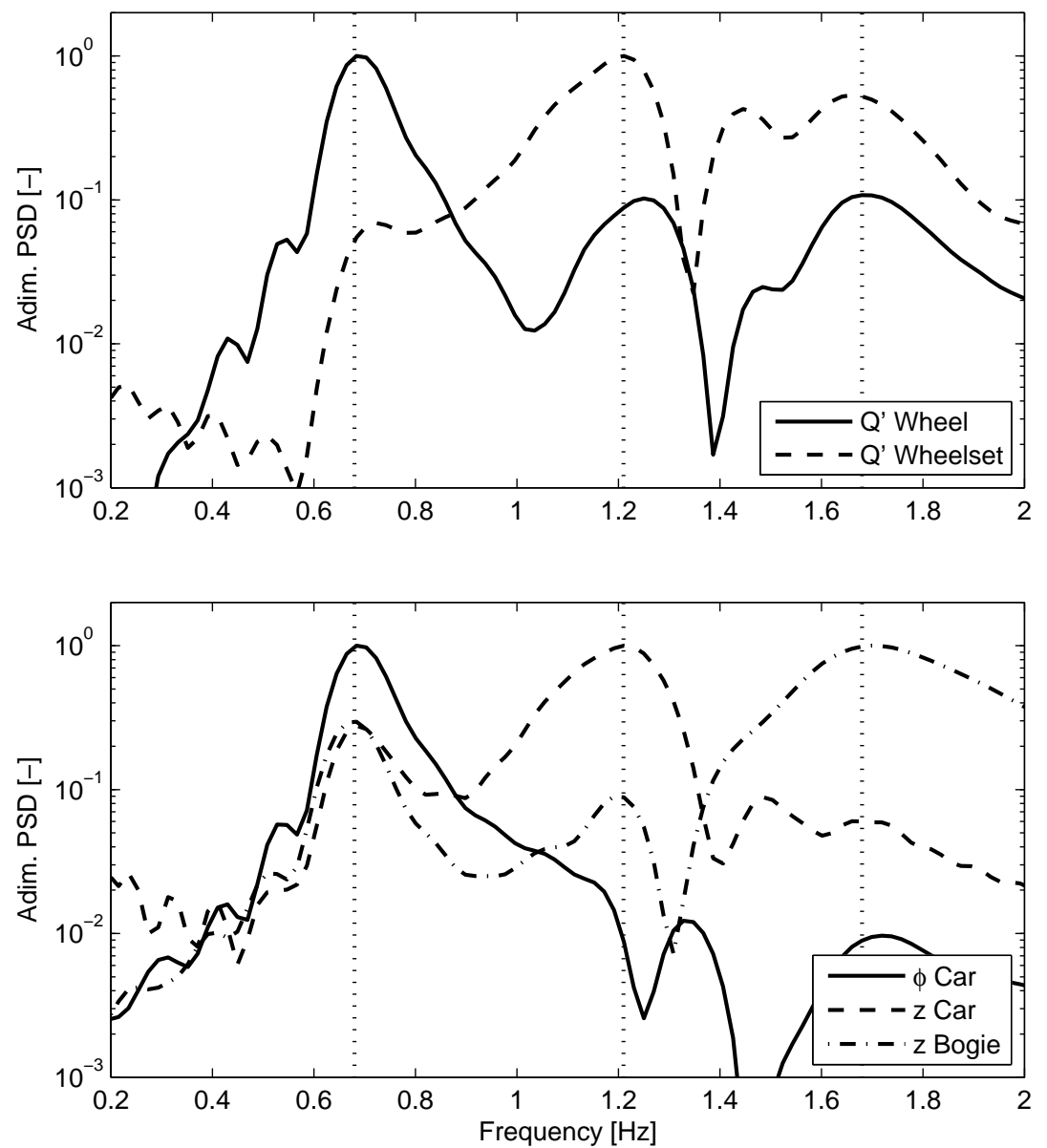

Figure 2.11: Power spectral density of the gust reponse (step gust) computed with the multibody model: wheel and wheelset unloading (top) and selected motions (bottom). The dotted lines mark the frequency of the peaks.

approximations, the most important being multibody dynamics (MBS). The main difference to the aerodynamics is that the numerical methods for driving dynamics produce very accurate results with low computational effort.

\section{Multibody dynamics}

The application of multibody dynamics to vehicle system dynamics is discussed e.g. in [KS93, Sch97, KSA01]. Two main components of the generic analysis process can be identified, [KL94, Sha89]:

- Formalism. The formalism is the algorithm used to formulate the equations of motions. The differences between formalisms lie in the approach (symbolic, numeric), the choice of the coordinates (absolute, relative), the mechanical principle (D'Alembert, Lagrange). Special attention must be given to the presence of kinematic loops, as in the case of railway vehicles when the W/R contacts are modelled 


\section{Crosswind stability}

Table 2.2: Lower eigenmodes with corresponding eigenfrequency compared with the frequencies of the nonlinear gust response (cp. Fig. 2.11).

\begin{tabular}{l||c|c|c} 
& $\begin{array}{c}\text { eigenfreq. } \\
{[\mathrm{Hz}]}\end{array}$ & $\begin{array}{c}\text { freq. nonlin. } \\
{[\mathrm{Hz}]}\end{array}$ & $\begin{array}{c}\text { carbody } \\
\text { motion }\end{array}$ \\
\hline \hline lower swaying & 0.53 & 0.68 & $y, \phi$ \\
bouncing & 0.79 & 1.21 & $z$ \\
hunting (carbody) & 0.93 & highly damped \\
pitching & 0.97 & 1.21 & $\gamma$ \\
upper swaying & 1.47 & \multicolumn{2}{|c|}{ highly damped }
\end{tabular}

as constrains. Flexible bodies can also be considered by a modal representation derived from a finite element analysis. The resulting equations of motion usually form a differential algebraic system.

- Integration. Even though modern MBS codes can perform a wider range of analyses, the most common one is the computation of time histories. To this aim the equations of motion must be integrated in time. Special methods tailored to the resulting differential algebraic system have been developed; the most efficient and widespread integrator is the DASSL, which is an implicit multistep integrator with variable stepsize, [ESF98].

When conventional driving dynamics problems are studied, modern MBS codes completely automatise the analysis process so that the main task of the user is reduced to the model set-up. However, this task can be very challenging and in the end determines the quality of the results.

\section{Other methods}

For particular driving dynamics tasks, like stationary curving or stability analysis, special models are often used. The question arises whether the accuracy of multibody simulation is adequate for the relatively simple case of the crosswind stability; actually, not all the existing crosswind stability norms require multibody simulations or they only do for some classes of vehicles and driving velocity ranges. For example, in [DEST04] a static approach is discussed; the characteristic wind curve can be computed very rapidly but a special model must be provided and unsteady phenomena cannot be considered. 


\subsection{Parametric uncertainty/variability}

A different approach consist in simplifying an existing multibody model, as multibody models are always created in the design process to assess the general riding performances of new vehicles. In Sec. 3.1.1 the elimination of the W/R contact and the linearisation starting from the complete multibody model will be proposed and discussed.

\subsection{Parametric uncertainty/variability}

\subsubsection{Sources of uncertainty and variability}

Uncertainty is the result of a lack of knowledge. It originates mainly from:

- Intrinsic stochastic phenomena which can be well observed but reasonably modeled only by statistics. This is the case of the gust parameters.

- Phenomena that can only be studied and thus modelled in a simplified way, because of some technological limits. This is the case of the aerodynamic coefficients.

A partial solution to the problem of uncertainty is given when the risk assessment is based not on an absolute evaluation but on a relative one. This approach is used, for example, in the RIL401, where the performances of a reference vehicle are used as an indicator. On the other hand, this approach only works if the comparison is performed correctly. For example, when the aerodynamic coefficients of a new vehicle are measured, the coefficients of the reference vehicle should be acquired too, under identical conditions: same wind tunnel, same set-up, same model dimensions and refinement level, etc. This leads to a very large effort which is hardly bearable.

Even if uncertainty were absent and perfect complete models available, the values of the corresponding parameters would be needed for the risk analysis. This is the problem of variability. Although some phenomena can be monitored easily (air temperature, wind velocity at single points, etc.), others, like wind velocity profile or transient phenomena, cannot. It is only reasonable to make simplifying assumptions and model the variability in a nondeterministic way.

\subsubsection{Models for uncertainty}

As already explained in Sec. 1.2 the uncertainty/variability is taken into account in the proposed approach by modelling the affected parameters as stochastic variables. Problematic is the handling of the aerodynamic coefficients because they are not scalars but functions of the yaw angle $\theta$. Three possible modelling solutions for the description of the generic coefficient $C$ are:

- Stochastic process. The most accurate way to model the coefficients is to handle them as stochastic processes. Accordingly, the coefficient $C$ should be expressed as a function $C(\theta ; \zeta), \zeta$ being the outcome of an ideal experiment, see for example [Pap02]. For this model, a huge amount of statistical information is needed and the resulting computational effort cannot be reasonably borne. Moreover, it is questionable whether the very high level of accuracy that is achieved is appropriate. 


\section{Crosswind stability}

- Set of stochastic variables. In a less accurate approach the generic coefficient can be modelled as a function of $\theta$ and a finite set of stochastic variables grouped in the vector $\mathbf{X}$. According to the fact that, in practice, the coefficients are known (measured or computed) only at few yaw angles, the variables $X_{i}$ are chosen as the values of the coefficient $C$ at such yaw angles, e.g. $\bar{\theta}_{1}=5^{\circ}, \bar{\theta}_{2}=10^{\circ}$, etc. The coefficient can then be expressed as:

$$
C(\theta ; \mathbf{X})=C\left(\theta ; C\left(\bar{\theta}_{1}\right), \ldots, C\left(\bar{\theta}_{m}\right)\right) .
$$

For a given outcome of the set $\mathbf{X}$, the dependency of $C$ on $\theta$ reduces to an interpolation. If the number of variables is low, the approach is not expensive but accurate.

- Single stochastic variable. In the most basic approach a deterministic function describing the nominal coefficient, $C_{0}(\theta)$, is considered and the uncertainty is reduced to a single stochastic variable $X$ acting as a multiplicative factor. It is convenient to choose $X$ as the value of the coefficient for a significant yaw angle, e.g. $\bar{\theta}=20^{\circ}$ for the reasons explained in Sec. 2.2.1. It follows:

$$
\begin{aligned}
C(\theta ; X) & =\frac{X}{C_{0}\left(20^{\circ}\right)} C_{0}(\theta) \\
E[X] & =C_{0}\left(20^{\circ}\right)
\end{aligned}
$$

This approach is very simplifying but allows scalar parameters and functions to be equally handled, because the uncertainty is modelled for both of them by a single stochastic variable.

For the sake of simplicity the third approach (single stochastic variable) was chosen for the work but all the methods and techniques described and used in this work could have been directly applied to the model expressed by (2.16).

\subsubsection{Stochastic variables}

Table 2.3 summarises the distributions used in this work. They are briefly discussed in the following sections.

Normal and Lognormal Because of its suitability as a model for many physical phenomena, the normal distribution is widely used in engineering. This distribution is commonly adopted when statistic information about a variate is limited to the second order moment. For variates defined only by lower and top bound it is common to assume a gaussian distribution and to interpret the bounds as the $\pm 3 \sigma$ limits. The normal distribution has a relevant role in reliability analysis because most of the semianalytic techniques that will be described in Sec. 4 are exact only for normal variates.

The lognormal distribution should be used instead of the gaussian when the parameters cannot physically assume negative values. The properties of the lognormal and 
Table 2.3: Distributions referenced in this work.

\begin{tabular}{|c|c|c|c|}
\hline & $\operatorname{PDF} f_{X}(x)$ & Mean & Variance \\
\hline Normal & $\frac{1}{\sqrt{2 \pi \sigma^{2}}} e^{-(x-\mu)^{2} / 2 \sigma^{2}}$ & $\mu$ & $\sigma^{2}$ \\
\hline Lognormal & $\frac{1}{x \sqrt{2 \pi \sigma^{2}}} e^{-(\ln x-\mu)^{2} / 2 \sigma^{2}}, x \geq 0$ & $\mu$ & $\sigma^{2}$ \\
\hline Rice $^{a}$ & $\frac{x}{\sigma^{2}} e^{-\left(x^{2}+V^{2}\right) / 2 \sigma^{2}} I_{0}\left(\frac{|V| x}{2 \sigma^{2}}\right)$ & See [ & ap02] \\
\hline Rayleigh & $\frac{x}{\sigma^{2}} e^{-x^{2} / 2 \sigma^{2}}, x \geq 0$ & $\sqrt{\frac{\pi}{2}} \sigma$ & $(2-\pi / 2) \sigma^{2}$ \\
\hline Type I extreme & $\begin{array}{l}a e^{-\alpha(x-u)} \exp \left(-e^{-\alpha(x-u)}\right) \\
x \geq 0, \alpha>0, u>0\end{array}$ & $u+\frac{0.5772}{\alpha}$ & $\frac{1}{6} \frac{\pi^{2}}{\alpha^{2}}$ \\
\hline Uniform & $\left\{\begin{array}{cl}0 & x<a, x>b \\
\frac{1}{b-a} & a \leq x \leq b\end{array}\right.$ & $\frac{b+a}{2}$ & $\frac{1}{3}\left(\frac{b-a}{b+a}\right)^{2}$ \\
\hline $\begin{array}{l}\text { Triangular } \\
\text { (symmetric) }\end{array}$ & $\left\{\begin{array}{cl}0 & x<a, x>b \\
\frac{x-a}{(b-a)^{2}} & a \leq x \leq(b+a) / 2 \\
\frac{b-x}{(b-a)^{2}} & (b+a) / 2<x \leq b\end{array}\right.$ & $\frac{b+a}{2}$ & $\frac{1}{6}\left(\frac{b-a}{b+a}\right)^{2}$ \\
\hline
\end{tabular}

${ }^{a}$ In the rician PDF, $\sigma$ and $V$ are parameters and $I_{0}$ is:

$$
I_{0}(x)=\frac{1}{2 \pi} \int_{0}^{2 \pi} e^{x \cos \varphi} \mathrm{d} \varphi
$$

${ }^{b}$ Also called Fisher-Tippett distribution. 


\section{Crosswind stability}

gaussian distributions are similar and the seminanalytical methods for the reliability analysis can be applied to lognormal variables. Nevertheless, as long as the ratio $\mu / \sigma$ is large, which is the case of the parameters studied in this work, both distributions are very similar and the use of lognormal variables instead of normal ones would not have any significant influence on the results.

Rice and Rayleigh The Rice distribution characterises the distribution of the local maxima of a Gaussian stochastic process, [WPO95, LBH03b]. For a process having variance $\sigma^{2}$ the PDF is reported in Table 2.3; the parameter $V$ is connected with the bandwidth of the spectral power density of the process.

The Rice distribution approaches a Rayleigh distribution for narrow banded processes, whereas for wide banded processes it tends asymptotically to a Gaussian distribution, i.e. it is identical with the distribution of the original stochastic process. The rician PDF can also be expressed as the sum of a Normal and a Rayleigh PDFs weighted with the bandwidth parameter $V$.

Type I extreme value In general, asymptotic extremal distributions are used to describe the distributions of the maxima (or minima) from a large number of samples. Wind climatology makes large use of such distributions, each sample being composed of measurements of the wind velocity over a fixed period, usually one year. For this application the Type I extreme value distribution is commonly chosen, [Rus82]. In the expression of the PDF, $\alpha$ is a parameter connected with the dispersion of the data and $u$ is the modal value, i.e. the most probable value. In wind climatology both parameters depend mainly on the location but also on other factors like height over ground etc.

Uniform The uniform distribution is sometimes used when only lower and upper bounds for the variate are available. The choice of this distribution is close to a deterministic "worst-case" approach. It must be noted that a uniformly distributed variate is bounded, i.e. the possible outcomes cover only a subset of $\mathbb{R}$.

Triangular The triangular distribution is often adopted in engineering applications. It has been used in some sections of this work only as a reference to show how the results are influenced by the choice of the distribution of the variables. The triangular distribution, like the uniform, is bounded.

\section{Dual representation}

When modelling an uncertain/variable parameter as a single stochastic variable it is not possible to discern uncertainty and variability. A slightly enhanced representation is the dual one, see for example [AT84], which consists in a separation of the two effects by using two stochastic variables for every parameter. The approach has not been used in this work and is only reported for completeness. 


\subsection{Parametric uncertainty/variability}

If the parameter $p$ of interest has nominal value $p_{0}$, the dual representation can be expressed as

$$
\begin{aligned}
& P=U+V \\
& E[U]=p_{0}, E[V]=0 .
\end{aligned}
$$

The random variables $P, U$ and $V$ represent respectively the parameter, the contribution due to uncertainty and the contribution due to variability. This model can also be interpreted as a stochastic variable (variability) with an expected value being again a stochastic variable (uncertainty):

$$
E[P \mid U]=U
$$

The dual representation requires more statistical information about each parameter but, on the other hand, it allows investigating separately the effects of uncertainty and variability. Even if solid stochastic information is rarely available, it is in general possible to decide qualitatively whether a parameter is mainly affected by uncertainty, variability or both. Moreover, it must be observed that if $V$ and $U$ are modelled as gaussian variables $P$ is also a gaussian variable with $\mu_{P}=\mu_{V}+\mu_{U}$ and $\sigma_{P}^{2}=\sigma_{V}^{2}+\sigma_{U}^{2}$. That is to say, the dual representation does not introduce any additional complexity in the analysis. 


\section{Modelling and simulation}

\section{Chapter overview}

Fig. 3.1 summarises the modelling and simulation workflow and gives an overview on this chapter. First of all the vehicle model is considered: it consists of the mechanical model and the aerodynamic model. A German ICE2 driving trailer was taken, [MR97], because
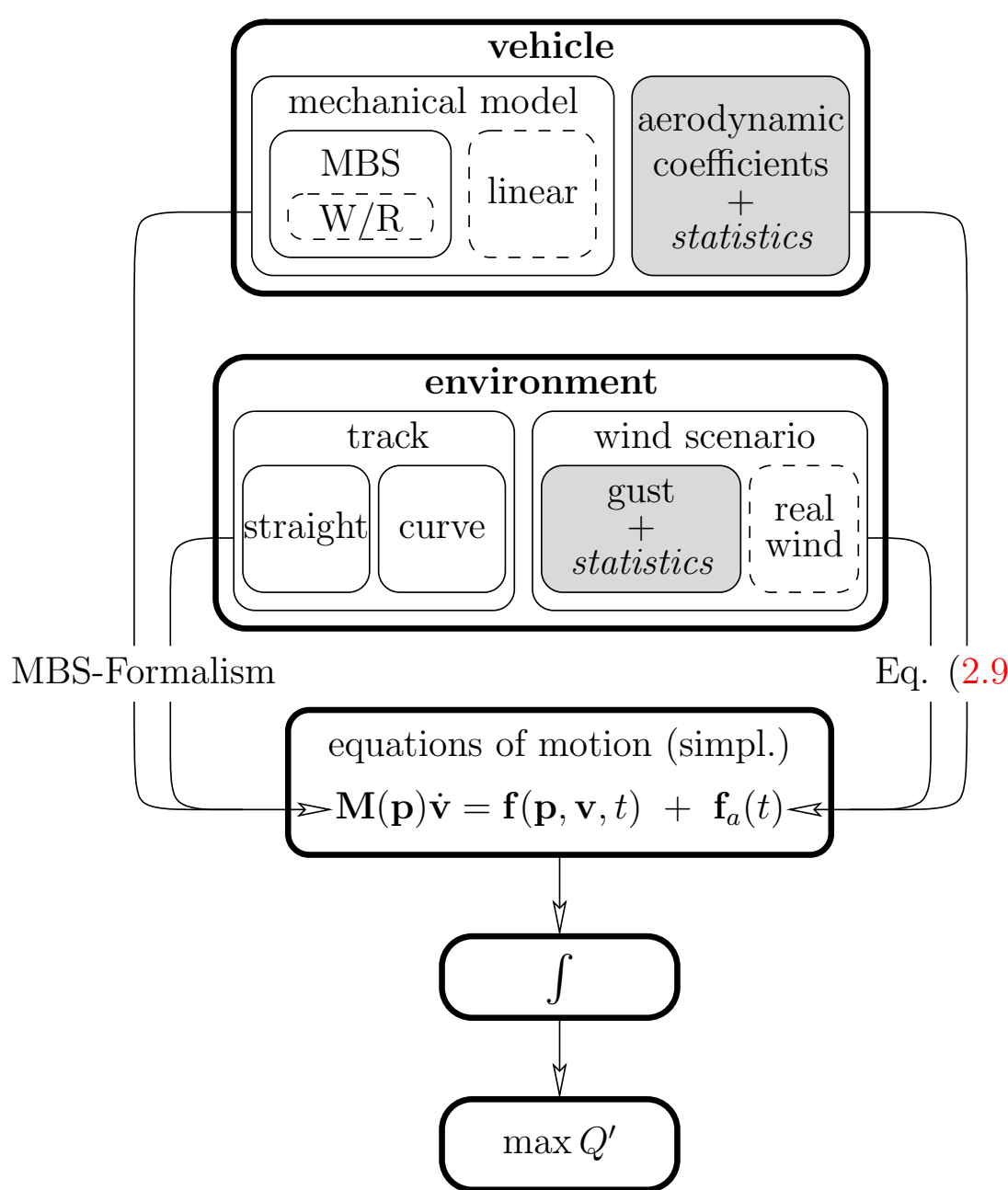

Figure 3.1: Overview of the modelling elements within the simulation workflow discussed in this chapter. Dashed boxes denote proposed modification to the conventional models, shaded boxes the parts affected by parametric uncertainty. 
in Germany this vehicle has evolved to a kind of benchmark. The mechanical model is a multibody model which also includes the $\mathrm{W} / \mathrm{R}$ contacts. An alternative model without $\mathrm{W} / \mathrm{R}$ contacts and a linear model will be discussed too. In this work the aerodynamic model of the vehicle is composed only of one set of aerodynamic coefficients but in general it could comprehend many coefficients sets to cover different environmental conditions, one or more aerodynamic admittance functions etc. Moreover, the statistics of the coefficients describing the uncertainties are defined. Such statistics are not directly used in the simulation workflow because in every simulation run the coefficients assume fixed values; the statistics are used by the reliability framework that directs the simulations, see Sec. 4.

The environment model includes the track and the wind scenario. The former consists of straight track and curve with different cant deficiencies; the latter is an ideal gust, for which the statistics describing the uncertainty are given. Again, the statistics are part of the model; they are not used in the simulation process but in the reliability framework. A scenario based on real measured wind samples is also briefly addressed.

The elements defining the mechanical part of the model, i.e. vehicle and track, establish by means of the MBS formalism, [Sha89, KL94], the left hand side of the equations of motion and a part of the right hand side forces, whereas the elements defining the aerodynamic model contribute only to the right hand side according to Eq. (2.9). In Fig. 3.1, where it was assumed for the sake of simplicity that kinematic loops are not present, $\mathbf{M}$ is the mass matrix, $\mathbf{p}$ the position coordinates, $\mathbf{v}$ the velocities, $\mathbf{f}$ the generalised forces and $\mathbf{f}_{a}$ the aerodynamic forces. In the used model the aerodynamic forces $\mathbf{f}_{a}$ are only function of time but not of the coordinates.

Finally, the equations of motion are integrated; the maximal value of the wheel unloading is extracted from the time history and passed to the reliability analysis. For the multibody modelling and simulations the tool SIMPACK has been used; it is was originally developed at the DLR and is now commercially available, [RE93, SNMG99].

\subsection{Vehicle model}

\subsubsection{Mechanical model}

\section{Multibody model}

The used multibody model is composed of 11 bodies and 98 force elements and has 37 d.o.f. (45 joint states and 8 constraints representing the $\mathrm{W} / \mathrm{R}$ contacts). As only low frequencies are relevant the carbody is modelled as rigid. Further on, as overturning and not derailment is the phenomenon of interest, the bogies are modelled as rigid too; their flexibility would be important if derailment were considered. The wheel rail contact, which is in general the core of a railway model, does not play a central role because an ideal wind/track scenario is used, as discussed below, see Sec. 3.2. It follows that there is no lateral motion between wheelsets and rail, the wheelsets assuming constantly the maximal lateral displacement towards the lee side. Moreover, even though flange contact occurs on the lee side, a one contact point model can be used because only the forces on 


\section{Modelling and simulation}

the wind side are relevant for the unloading criterion. Such a model is significantly less computationally expensive than a multiple contact point one. Finally, the only elements which need to be modelled accurately are the nonlinearities and the bump stops that limit the lateral displacement between car and bogies.

Even if a simulation of the complete overturning accident is technically possible this is not sensible as long as the unloading criterion is used. In this case the simulation has to be stopped when wheel lift is reached, because the unloading indicator stops working $\left(Q^{\prime}=1\right.$ independently of the severity of the wheel lift, cp. Eq.(2.15)). An interesting alternative, which well suits to the limitations of the unloading criterion and has been used in this work, is to define the $\mathrm{W} / \mathrm{R}$ contact as bilateral constraint. In this way traction forces can be obtained and the overturning risk can be somehow quantified also in a range which is not covered by the conventional approach $\left(Q^{\prime}>1\right)$. It is obvious that in doing so caution is needed and only values of $Q^{\prime}$ slightly greater than 1 , corresponding to little wheel lift, can be accepted.

\section{Multibody model without $\mathrm{W} / \mathrm{R}$ contact}

As only overturning is considered, the question of the influence of the $\mathrm{W} / \mathrm{R}$ contact model on the wheel unloading arises. To ascertain this fact a multibody model has been set up in which the $W / R$ contact was substituted by a rheonomic joint. It follows that the wheelsets do not rotate but are guided, at the vehicle driving velocity, in their nominal position in the middle of the rails.

The results of a test simulation with the model without W/R are reported in Fig. 3.2, top, and are compared with the results computed with the complete model. A real wind scenario was used for the test because it is more demanding than an ideal gust; moreover, the final aim is to create a linear model which should work with real wind scenarios as discussed below. It can be seen that the agreement is very good, although the peak values, e.g. around $t=65 \mathrm{~s}$, are slightly underestimated. The fact that, in general, higher frequencies seem to be missing can be ascribed to the suppressed lateral movement of the wheelsets. However, if the results computed with the complete model are low pass filtered (0.6 Hz was used), a good agreement of the peak values between complete model and model without W/R contact can be reached, Fig. 3.2, middle. Filtering is actually prescribed in the norms because overturning is a very low-frequency phenomenon. For example, the RIL401 requires low-pass filtering at $1.5 \mathrm{~Hz}$ for high quality track. When ideal track is used, as in Fig. 3.2, still lower values are reasonable and thus $0.6 \mathrm{~Hz}$ seems to be approapriate. However, the filtering frequency to be used is still a topic of discussion.

As to the computational effort, it is reduced after removing the $\mathrm{W} / \mathrm{R}$ contact by a factor $\simeq 5$. This is due to the fact that, when real wind load is used, the wheelsets perform continuous lateral displacements which must be followed and computed by the $\mathrm{W} / \mathrm{R}$ contact module. If an ideal gust is used the reduction factor of the computational effort is less than 2 because the wheelsets persist during the whole simulation in the maximal lateral displacement toward the lee rail. 

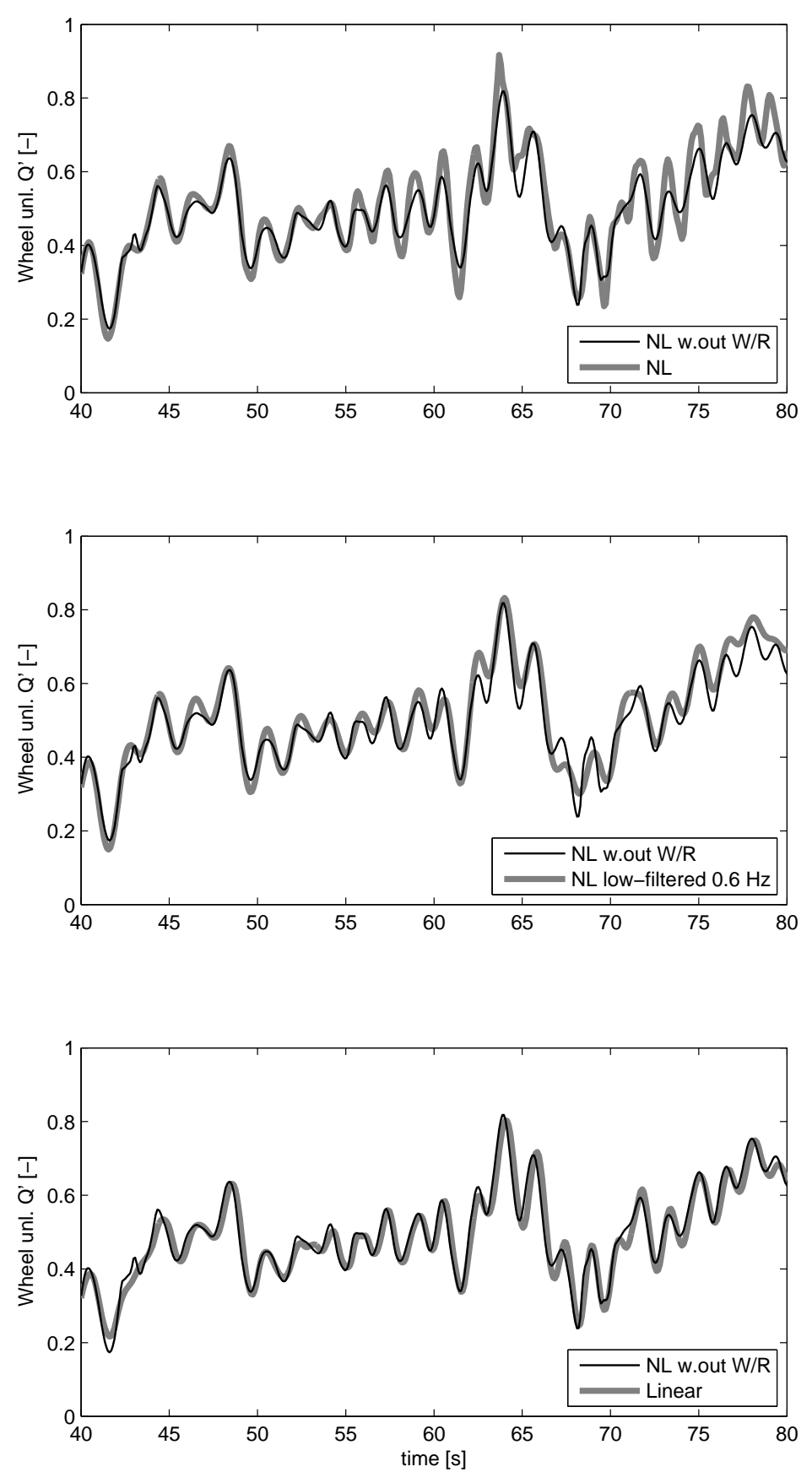

Figure 3.2: Comparison of wheel unloading time histories for real wind excitation (wind time history of Fig. 2.7, $U_{0}=20 \mathrm{~m} / \mathrm{s}$ ). Top: Nonlinear MBS model without $\mathrm{W} / \mathrm{R}$ contact and complete nonlinear MBS model. Middle: Nonlinear MBS model without W/R contact and complete nonlinear MBS model low pass filtered at 0.6 Hz. Bottom: Nonlinear MBS model without $\mathrm{W} / \mathrm{R}$ contact and linear model. 


\section{Modelling and simulation}

\section{Linear model}

The availability of a linear model of the vehicle would allow the use of a wide range of techniques that cannot be applied to the nonlinear model; especially real wind scenarios, instead of idealised gusts, could also be used, e.g. for covariance analysis. Moreover, a linear model would improve significantly the reliability evaluation, the uncertainty propagation etc., as will discussed in Sec. 4. Finally, if only the steady state response is of interest only a linear static problem would have to be solved instead of performing any time integration.

Commercial MBS codes can derive automatically a linearised model from a complete nonlinear one, the linearisation being usually performed around the nominal position; this is commonly done for stability analysis purposes. However, for the crosswind stability task it is only reasonable to linearise around the displaced position, i.e. the position assumed under the steady load corresponding to the mean wind $U_{0}$. On the other hand, a linearisation of the $\mathrm{W} / \mathrm{R}$ contact is not possible when the wheelsets are not in their nominal position, because of the strong irregularities in the $\mathrm{W} / \mathrm{R}$ geometry. To override this inconvenience, considering that the $\mathrm{W} / \mathrm{R}$ has been observed to be not influential, the model without $\mathrm{W} / \mathrm{R}$ can be used to produce the linear model.

To examine this fact a linear model was created from the model without $\mathrm{W} / \mathrm{R}$ contact at the position due to the aerodynamic load corresponding to the mean wind $U_{0}$. In Fig. 3.2, bottom, the wheel unloading time history computed by time integration of the linear model is compared with the result from the multibody model without $\mathrm{W} / \mathrm{R}$ contact. An almost perfect agreement can be observed, confirming the validity of the linear model. This model was not used or tested thoroughly in this work; its functionality for analysis techniques different from the time integration should be object of further work.

\subsubsection{Aerodynamic model of the vehicle}

As far as the aerodynamic model of the vehicle is concerned, two assumptions are always made in the crosswind stability norms:

- The motion of the vehicle except from the motion along the track has no influence on the aerodynamic loads. As the driving velocity in each simulation is constant, the aerodynamic loads result to be only a function of the wind angle and the crosswind velocity which is in turn a function of time or the track coordinate.

- The inclination of the vehicle in curves is neglected. This is a questionable assumption because a consequence of the inclination is that the shape of the car exposed to the wind, and thus the aerodynamic coefficients, change; in addition, the projected lateral surface become larger. It follows that the resulting aerodynamic loads change. Such effects should be considered at least for tilting vehicles because the total inclination (track cant and tilting) is relatively large. 


\subsection{Vehicle model}

\section{Aerodynamic coefficients}

The nominal value of the coefficients over the yaw angle is reported in Fig. 3.3 (the drag coefficient is not reported because it does not significantly affect the crosswind stability). The coefficients are computed with respect to the geometrical centre of the car projected on the track plane (top of rail) according to (2.9). As usual in Germany, the reference area is $A=10 \mathrm{~m}^{2}$, independently from the real dimensions of the vehicle; the roll, nick and yaw moment coefficients are adimensionalised with respect to $A \cdot l$ with $l=3 \mathrm{~m}$.

The uncertainty in the coefficients was modelled by a stochastic variables defining the value of the coefficients for $\theta=20^{\circ}$, as discussed in Sec. 2.4.2. Even though all coefficients could be modelled as stochastic variables, it is desirable to have the lowest possible number of variables to reduce the computational effort. According to experience and to the results of screening simulations, which will be reported in Sec. 4.2.4, only some parameters influence significantly the vehicle behaviour. In the end, also aiming to simplify the presentation and the discussion of the results, only the uncertainties in the lift and roll moment coefficients have been considered in this work: the former has the most influence on the wheel unloading whereas the latter is characterised by the largest variations, see below.

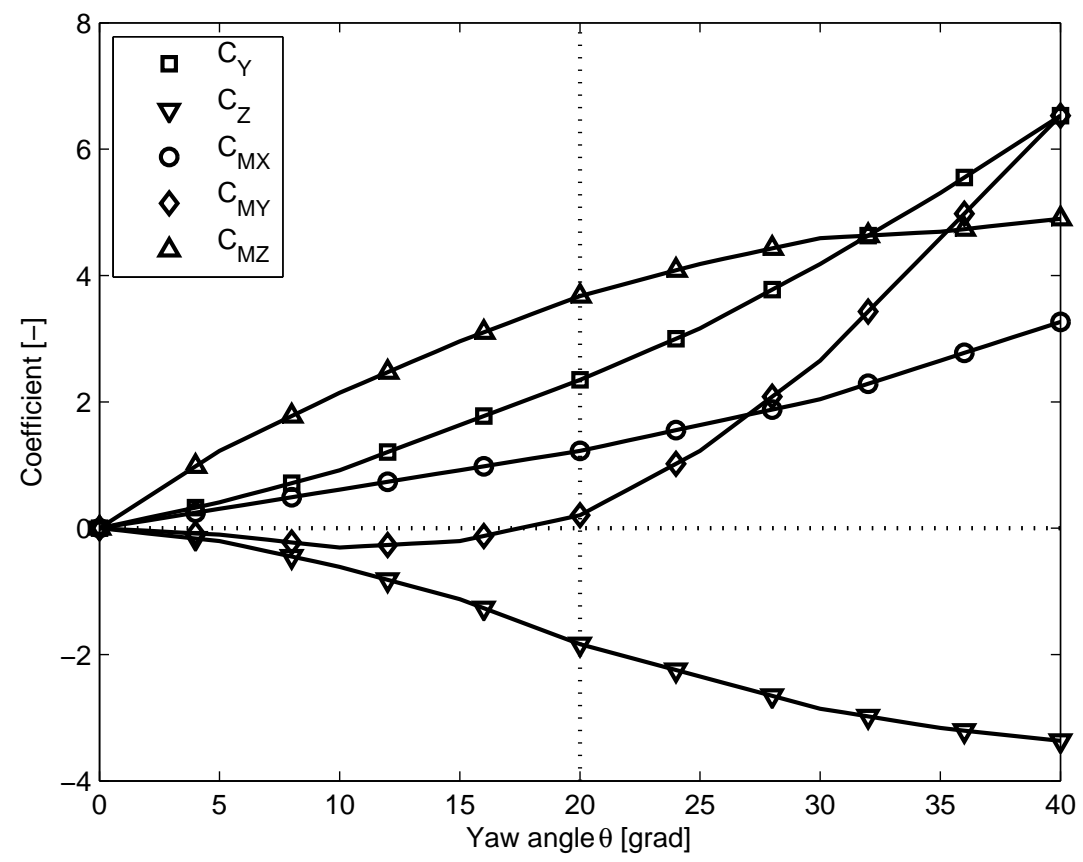

Figure 3.3: Aerodynamic coefficients of the ICE2 driving trailer.

\section{Statistics of the aerodynamic coefficients}

As a consequence of the lack of information a statistical description of the aerodynamic coefficients is a difficult task. Even when some homogeneous information is available, 


\section{Modelling and simulation}

the data is not sufficient for statistic inferences ${ }^{1}$. To this aim a literature survey was performed and is reported below. The gained qualitative information, together with the opinion of experts, was used to determine empirical bounds for the coefficients. The result is that an error bound of $20 \%$ for all coefficients except lift is reasonable. For the latter, very high error bounds must be expected; conservatively, a $40 \%$ error bound for the lift coefficient was chosen in this work. These bounds have been then interpreted as $\pm 3 \sigma$ limits for the gaussian distribution. As reported above, only the uncertainties in roll moment and lift coefficients have been considered in this work; they are summarised in Table 3.1.

The choice of the normal distribution is dictated by the absence of arguments for nonnormal distributions and is also advised in [SHB83, Kar88]. For comparison, a uniform distribution between the above mentioned bounds was also tested. A first attempt to step out from the poor statistical description will be made in Sec. 4.3, where the sensitivity analysis with respect to the statistics of the parameters is performed. The results will show which statistics are most influential, thus indicating to the aerodynamicists where a large amount of information is needed.

Table 3.1: Statistics of the aerodynamic coefficients considered as uncertain in this work.

\begin{tabular}{c||c|c|c|c} 
& distribution & $\mu$ & $3 \sigma$ & $(3 \sigma) / \mu$ \\
\hline \hline$C_{Z}\left(20^{\circ}\right)$ & $\begin{array}{c}\text { Normal } \\
\text { (uniform) }\end{array}$ & -1.84 & 0.72 & 0.4 \\
$C_{M X}\left(20^{\circ}\right)$ & $\begin{array}{c}\text { Normal } \\
\text { (uniform) }\end{array}$ & 1.22 & 0.24 & 0.2
\end{tabular}

\section{References}

Selected references, which motivate the value of the statistics reported above, are now mentioned and briefly discussed.

TRANSAERO Within the European project TRANSAERO, [SW+ $\left.{ }^{+} 2\right]$, a very extensive benchmark study on the aerodynamic coefficients of the German Inter-Regio driving trailer was performed. Most results, obtained with different techniques, lay within a relatively little error margin (e.g. $20 \%$ for $C_{M X}$ ) but agreement with the

\footnotetext{
${ }^{1}$ It must also be mentioned that efforts to establish a systematic framework for uncertainty quantification are in progress, e.g. [BGC04].
} 


\subsection{Vehicle model}

results of the corresponding full scale tests could not be reached. In general, the lift coefficient was affected by the largest uncertainty/variability.

Static tests A large amount of results of static wind tunnel tests are reported by many authors; however, they are not useful for the present work because they cannot be directly compared (different boundary conditions etc.). Worth mentioning are $\left[\mathrm{S}^{+}\right.$04], where peak/mean ratios larger than 2 were measured, and [OSB02], where the reduction of the coefficients by improvement of the vehicle shape is estimated to be $40 \%$ for $C_{Z}, 30 \%$ for $C_{M Z}$, and $20 \%$ for the other ones. Fluctuations in the coefficients due to vortex shedding (10\%) are described in [RRW04]. Moreover, large variations due to the wind/lee position for double track are reported in [CCDT03, STM03] (only high yaw angles were tested). Finally, in [Coo79] the consideration of an error bound of $15 \%$ for $C_{M X}$ is explicitly recommended.

Moving model test The necessity for moving model tests was discussed in Sec. 2.2.3 but agreement on this topic has not been reached yet. Older reports on moving model tests for railway vehicles can be found in [NS76, Coo81, How86]. In the latter work (1:5 moving model in real wind) scattering of the coefficients larger than $20 \%$ is measured but good agreement of the mean values with static wind tunnel is found. More recent test are reported in $\left[\mathrm{B}^{+} 03\right]$, where no differences between static and moving model tests for tunnel exit are observed.

Turbulent flow tests The effect of turbulence is probably the most studied and discussed topic; nonetheless, partially contradictory conclusions are drawn by different authors. In general the influence of the mean values seems to be low, whereas the influence on the peak values is large, even though not directly correlated to the velocity fluctuations ${ }^{2}$. In [KS94] the modelling of turbulence and boundary layer in wind tunnel tests of railway vehicles is explicitly recommended, because large influence on the maximal value of the coefficients is observed; different CWCs are also reported. In [CCDT03] the effects of turbulence on the CWC are studied too; the variations are reported to reach $100 \%$ of the overturning wind speed (details on the used risk analysis procedure are not given). On the contrary, in [Wil97, $\mathrm{B}^{+} 03$ ] no effect of turbulence is observed.

Modeling of underbelly The influence of the underbelly region on the coefficients, especially lift, seems to be very large, suggesting that it should be very accurately modelled in tests. In spite of it, this is rarely done because wind tunnel models are very small and details are difficult to recreate. Even if the underbelly is correctly modelled, the question of the local Reynolds number arises and the relative motion of the ground is not correct. For example, [Cop87] reports variations of $20 \%$ of the roll moment around the lee rail due to underbelly configuration. A comparison of measurements on models with and without bogies is reported in [Coo81]. A 100\% variation of lift due to bogies and front spoiler, computed by CFD, is reported in

${ }^{2}$ Recently turbulence effects have been extensively studied for road vehicles too, see for example [MG97]. 


\section{Modelling and simulation}

[RRW04]. Finally, in [Die03] the effect of relative ground motion is estimated by CFD to reach $20 \%$.

Full scale tests Full scale tests are very rare because of their cost and complexity. Very extensive tests on full scale staying vehicles were recently performed, [ $\left.\mathrm{B}^{+} 04\right]$, considering the effects of cant, turbulence, vehicle suspension etc. The results were compared with corresponding wind tunnel tests and good agreement $(\leq 20 \%$, except lift) was found. However, the importance of the modelling of the ground boundary layer in the wind tunnel was proved and comparison with older wind tunnel tests showed differences even over $50 \%$. Finally, peak/mean ratios up to 1.5 for $\theta<30^{\circ}$ were measured and the lift was proved to be extremely sensitive to surface roughness.

\subsection{Environment model}

\subsubsection{Track scenario}

Ideal track was used as prescribed, for example, in the TSI. Track irregularities complicate the evaluation of the wheal unloading because of the superposition of the effects of the ideal gust and the irregularities. For example, if real measured irregularities are used in the simulation, the position along the track of the ideal gust with respect to the irregularities can significantly influence the peak value of the wheel unloading. If the irregularities are produced from spectral data, many simulations with different irregularities samples have to be performed and the results statistically analysed.

For curved track, different cant deficiencies have been considered in this work. The track cant $c$ can be expressed as:

$$
c=\frac{s}{g} \frac{v^{2}}{r} \underbrace{-\frac{s}{g} a_{l}}_{\text {cant def. }},
$$

$s$ being the track span, $g$ the acceleration of gravity, $v$ the driving velocity, $r$ the curve radius and $a_{l}$ the uncompensated lateral acceleration. The second summand is the cant deficiency $c_{d}$ and is set, when $c$ and $v$ are fixed, by changing the curve radius $r$. A general discussion on the interaction between cant deficiency and crosswind stability for high driving velocities can be found in [Lin01].

\subsubsection{Aerodynamic model of the environment}

The ideal gust model based on infinite correlation, Fig. 2.8 b"), was introduced in Sec. 2.2.2. According to such model, the shape of the ideal gust, its parameters and their statistics can be determined from wind measurements at a fixed point on the track. 


\subsection{Environment model}

\section{Gust shape}

In practice, different gust shapes are used. The most common are:

- "1 - cos". This gust shape is very popular because prescribed in almost all aeronautical norms, [Hob88], and also in the norms for wind energy plants, [IEC93].

- Exponential. It is also called mean gust because it is not empirical but obtained from the analysis of real wind data, [LBH03a]. This shape is the most advanced model available at the moment and is used in the TSI.

- Ramp. This shape was used in the RIL401, but also in other works and norms, [Lip99]. Additionally to its simplicity, the ramp shape is interesting because it can be used as an approximation of the aerodynamic load at tunnel exit if the rise time is chosen as the time the vehicle needs to pass the tunnel portal.

- Step. It is the simplest gust form and is often used because of its mathematical properties; it allows an analytical study of the vehicle response for linearised equations of motion, see e.g. [Huc98] for road vehicles.

Accepting the assumption of quasi-steady aerodynamics, the gust shapes discussed above could be used to compute directly the resulting aerodynamic loads by Eq. (2.9). This is done, for example, in the TSI and in the RIL401. However, there is no evidence that this approximation is correct; on the contrary, the very few available experimental results show that the unsteady (transient) aerodynamic effects can have a large influence on the time history of the aerodynamic loads.

To check this fact, the aerodynamic admittance measured by [HE83] and plotted in Fig. 2.5 on page 19 was used to compute the unsteady side force due to the gust shapes described above. The results are reported in Fig. 3.4 for impulse and step-type gusts, showing that the force is far from following the gust shape, as would do according to the quasi-steady approach. On the contrary, a very smooth developing of the loads corresponds to all gust shapes.

A simple remedy consists in using the quasi-steady approach having substituted the original, e.g. exponential, gust shape with a fictitious shape which resembles the time history of the resulting aerodynamic load. From Fig. 3.4 it can be deduced that an accordingly scaled "1-cos" shape is appropriate to this aim. That is to say, quasi-steady aerodynamics is assumed and the effects of transient aerodynamics are deferred to the correspondingly modified gust profile. In doing so, Eq. (2.9) can still be used and, even though the wind gust and the coefficients loose part of their physical meaning, closer to reality aerodynamic loads are obtained.

The dramatically expensive requisite for this approach is the availability of the aerodynamic admittance of all aerodynamic loads (Fig. 3.4 refer only to the side force). Even though the "1-cos" shape is likely to be be valid for all loads, the parameters (amplitude and period) will probably vary, because the physical phenomena (vortex shedding etc.) governing each load are partially different. Some experimental evidence of this fact can be found for example in [NS76, HE83], where aerodynamic lift and 


\section{Modelling and simulation}
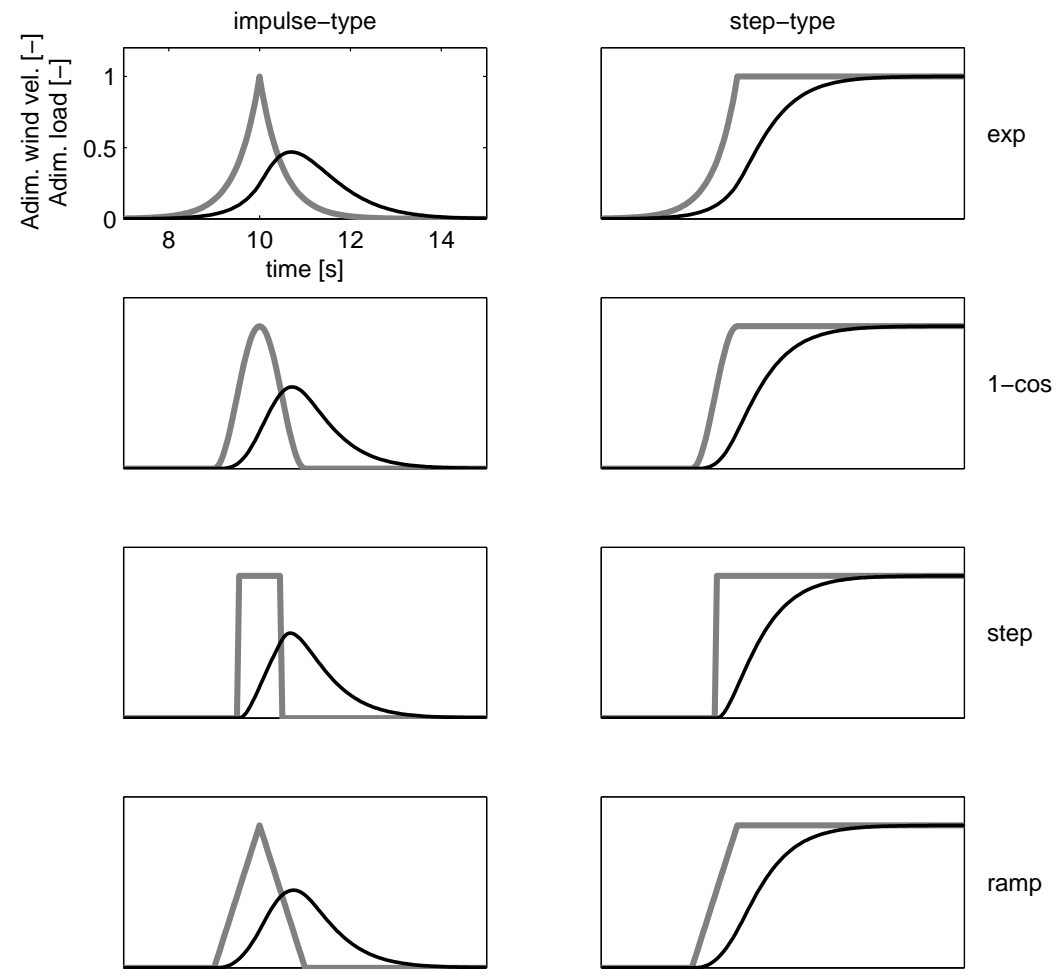

Wind $\longrightarrow$ Aerod. load

Figure 3.4: Time histories of different gusts with corresponding aerodynamic side force, both adimensionalised. Qualitative results according to admittance of Fig. 2.5.

side force are examined. However, for the sake of simplicity, such differences have been neglected in this work: the same fictitious gust profile, i.e. the same gust parameters, has been used for all loads.

To sum up, in this work the quasi-steady approach with was used with a smoothed gust profile ("1-cos") instead of the exponential one.

\section{Gust amplitude (gust factor)}

The gust factor is defined as the ratio between peak and mean value of the wind velocity:

$$
\text { Gust } F=\frac{U_{\max }}{U_{0}} .
$$

According to the previous section the gust factor of the fictitious smoothed gust has to be determined. Fig. 3.4 suggests that the factor should be reduced to avoid an overestimation of the real loads ${ }^{3}$. On the other hand, such results refer to the side

\footnotetext{
${ }^{3}$ Note that in Fig. 3.4 it is $U_{0}=0$ for simplicity's sake. Eq. (3.2) should be correspondingly reformulated.
} 


\subsection{Environment model}

force, whereas some experimental results suggest that for other aerodynamic actions the behaviour can be different and even overshoots (real aerodynamic loads larger than those arising from the quasi-steady approach) were measured. For this reason the gust factor of the fictitious gust has been assumed to be identical to that of the real wind gust.

In the RIL401 and in the TSI fixed values GustF $=1.8$ and GustF $=1.7$ are respectively prescribed. If compared with building aerodynamics norms, such values are located in a reasonable range. The value $G u s t F=1.8$ was thus adopted in this work as mean of the stochastic variable describing the gust factor.

When a complete statistical description is looked for, the theory of stochastic processes has to be used, [Dav64, LBH03b]. The local maxima of a stochastic process follow the Rice distribution, which was presented in Sec. 2.4.3. According to its properties, a gaussian distribution is an acceptable approximation and is equivalent to neglecting the Weibull part of the Rice distribution. This choice leads to a significant simplification of the reliability techniques and was thus adopted in this work. However, methodologies for the handling of nonnormal variables in the reliability analysis will also be presented, so that the rician distribution could also be used.

With regard to the variance, it can be observed that the variance of the normal part of the Rice distribution is the variance of the random process itself. Thus, as the Rice distribution was approximated by its normal part, this variance should be used. However, taking the variance of the wind as the variance of the peaks is physically not reasonable, because very large fluctuations would be produced. Moreover, the gust factor is a very influential parameter, as will be shown, for example, by the results of screening simulations in Sec. 4.2.4. For these reasons, also considering that an exact modelling of the wind process goes behind the scope of this work, it was conservatively set $3 \sigma_{\text {GustF }}=0.1 \cdot$ GustF .

\section{Gust length (duration)}

According to the used gust model, see Fig. 2.8 on page 24, the duration of the gust, Gust $\Delta t$, seen by a fixed observer on the track has to be specified. In the RIL401 a ramp gust having a duration of $1 \mathrm{~s}$ is prescribed. On the contrary in the TSI the gust is exponential and impulse-type, the duration being derived from the expected frequency of the wind. This frequency corresponds to the zero-up crossing frequency, [WP 095], and is often used for wind related problems, e.g. [Ber87]. It is given by the Rice's formula and for gaussian processes, like the wind, it can be computed using only the power spectral density. Finally, the expected gust length is:

$$
\text { Gust } \Delta t=\frac{1}{2 \omega_{0^{+}}}=\frac{1}{2}\left(\frac{\int_{-\infty}^{\infty} S_{U}(\omega) \mathrm{d} \omega}{\int_{-\infty}^{\infty} \omega^{2} S_{U}(\omega) \mathrm{d} \omega}\right)^{1 / 2}
$$

$S_{U}(\omega)$ being the PSD of the wind (e.g. von Karman spectrum). From this value, which refers to the general gust, the mean duration of the extreme gust is derived using the 


\section{Modelling and simulation}

so-called normalised gust amplitude. For this parameter a fixed value is given in the TSI.

More strictly the problem has to be formulated as a first-passage problem for the first derivative of the velocity: given the time when $\dot{U}=0$, the time has to be found when the next zero crossing of the velocity occurs. For this task no exact solutions are available and some approximations have been proposed, see for example [WP095]. In this work no effort was made in this direction because it would go beyond the prefixed scope. Even if the resulting distribution is likely to be an extreme related one like Rayleigh's or Weibull's, a normal distribution was basically used. This is acceptable because the Rayleigh distributions is very similar to the normal or lognormal distribution for wide ranges of the parameters.

For the choice of the mean, Eq. (3.3) cannot be directly applied because it refers to the exponential shape, whereas in this work the " 1 - cos" shape is used, as discussed above. A value of $2 \mathrm{~s}$ was empirically chosen, leading in the end to a gust which is comparable with the TSI gust and is also congruent with the RIL401 (in the RIL401 a step gust is considered, i.e. the length is halved).

The choice of the variance of the gust length is made difficult by the above mentioned problem that exact solutions do not exist. On the other hand, the gust duration will result not to be an influential parameter. Having qualitatively estimated that large fluctuations of the gust length can arise in practice as suggested, for example, by the recent results from the NewGust model, [LBH03a], the value $3 \sigma_{G \Delta t}=0.5$ Gust $\Delta t$ was chosen. Further work must be obviously done in this direction.

\section{Final crosswind scenario}
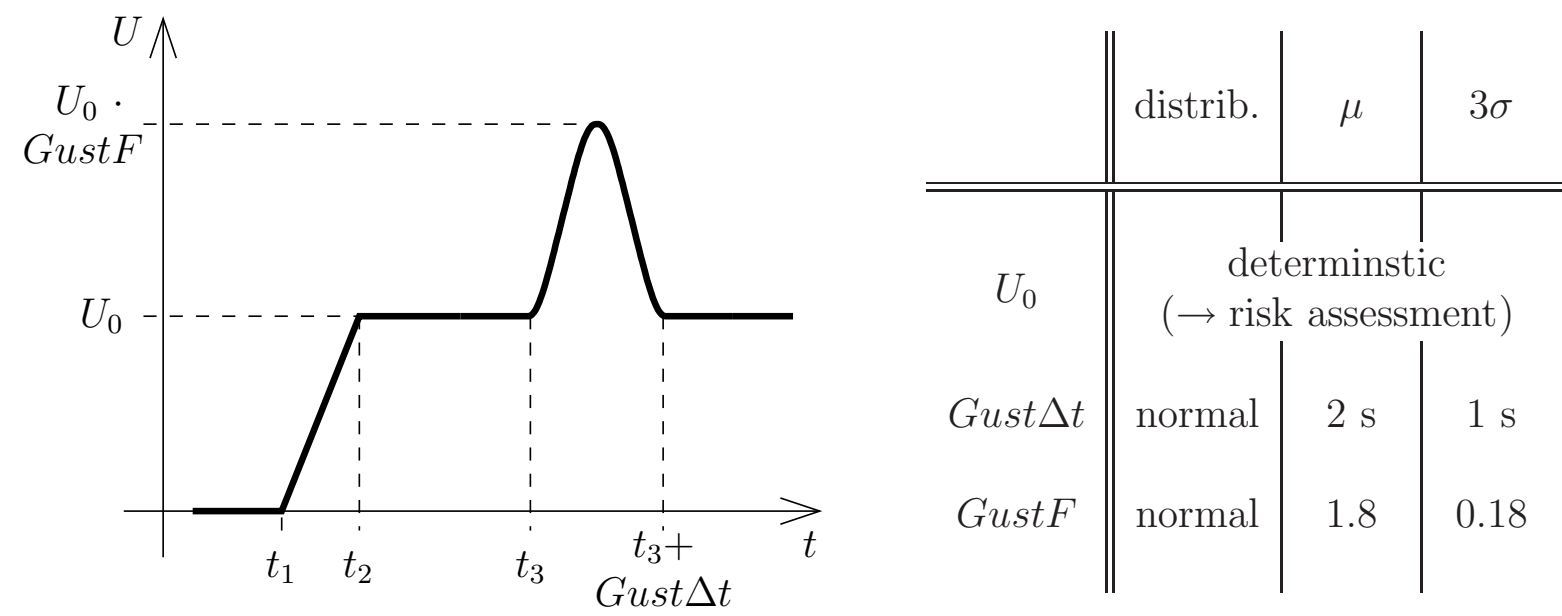

Figure 3.5: Crosswind scenario with " $1-\cos$ " gust used in this work. Note that $U_{0}$ is a stochastic variable in the risk assessment but not in the risk analysis, see Sec. 2.1.3.

The definitive crosswind scenario is plotted in Fig. 3.5, where the statistics are also summarised. It is composed of a constant mean velocity $U_{0}$ and the ideal gust which has a duration $\Delta t$ and a maximum $U_{0} \cdot$ Gust $F$. To simplify the simulations the crosswind 


\subsection{Simulation}

velocity is initially zero and rises afterwards to $U_{0}$ with a ramp. In this way the vehicle is unloaded when the simulation starts and the nominal states can be used as initial states; otherwise the static equilibrium corresponding to $U_{0}$ should be previously computed.

Two general assumptions about the wind direction are done:

- The wind direction is always perpendicular to the track. This means that when driving on a curve the direction of the wind is changed with respect to the inertial reference system.

- The wind direction is always parallel to the track plane. This assumption is quite strong because the fact that on a bridge or embankment the wind blows locally from the bottom up is neglected. However, in order to model this fact, aerodynamic coefficients as a function of the roll angle would be needed.

\subsection{Simulation}

In Fig. 3.6 the result of a typical MBS simulation, computed by SIMPACK, is plotted; the gust parameters have been assigned their mean (nominal) values. From the wheel unloading time history only the peak value, $Q^{\prime}=0.72$ in the example, is extracted and returned to the reliability analysis. The large overshoot induced by the gust must be stressed, documenting the importance of unsteady phenomena and thus the inadequacy of approaches based on static equilibrium.

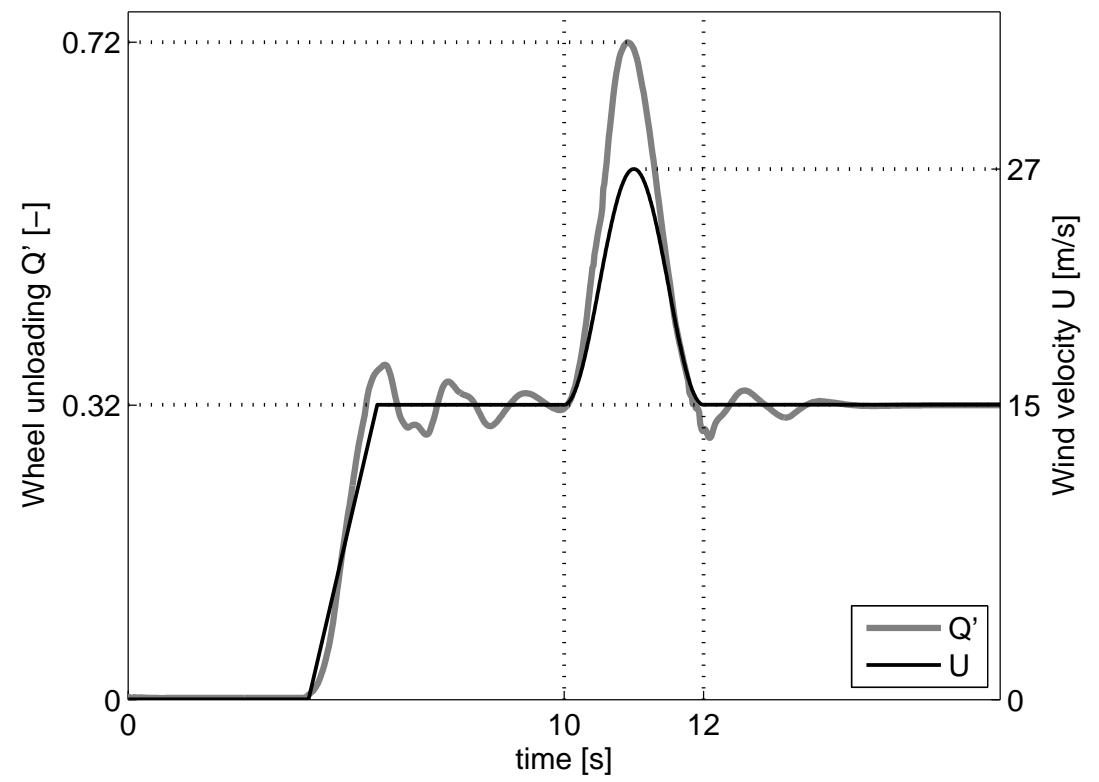

Figure 3.6: Result of a typical MBS simulation.

As far as the numerics is concerned, only the constraints representing the $\mathrm{W} / \mathrm{R}$ contact are a challenge for the integrator because the model does not include special elements like control loops etc. However, modern integrators are tailored to the stiffness 


\section{Modelling and simulation}

of the equations resulting from the $\mathrm{W} / \mathrm{R}$ contact. Moreover, as only rigid bodies and one point contact have been used, the computational effort for every simulation is little ( $\simeq 15$ s on a typical workstation).

The results of the simulations have not been filtered. It has been already discussed that the RIL401 prescribes low pass filtering with $1.5 \mathrm{~Hz}$ for good track quality. Such a filtering is only effective if real wind scenario and/or track irregularities are used, as in Fig. 3.2, but for ideal gust scenario it does not influence the results. On the contrary, filtering with very low cutoff frequencies, motivated by the fact that overturning is a low frequency phenomenon, would affect the results. However, the choice of the frequency should be object of deep investigation. It must be noted that RIL401 also specifies to perform an additional postprocessing by taking the 99.85 percentile of the filtered data as definitive peak value. For the same reasons adduced above this was not considered. 


\section{Reliability evaluation}

\section{Chapter overview}

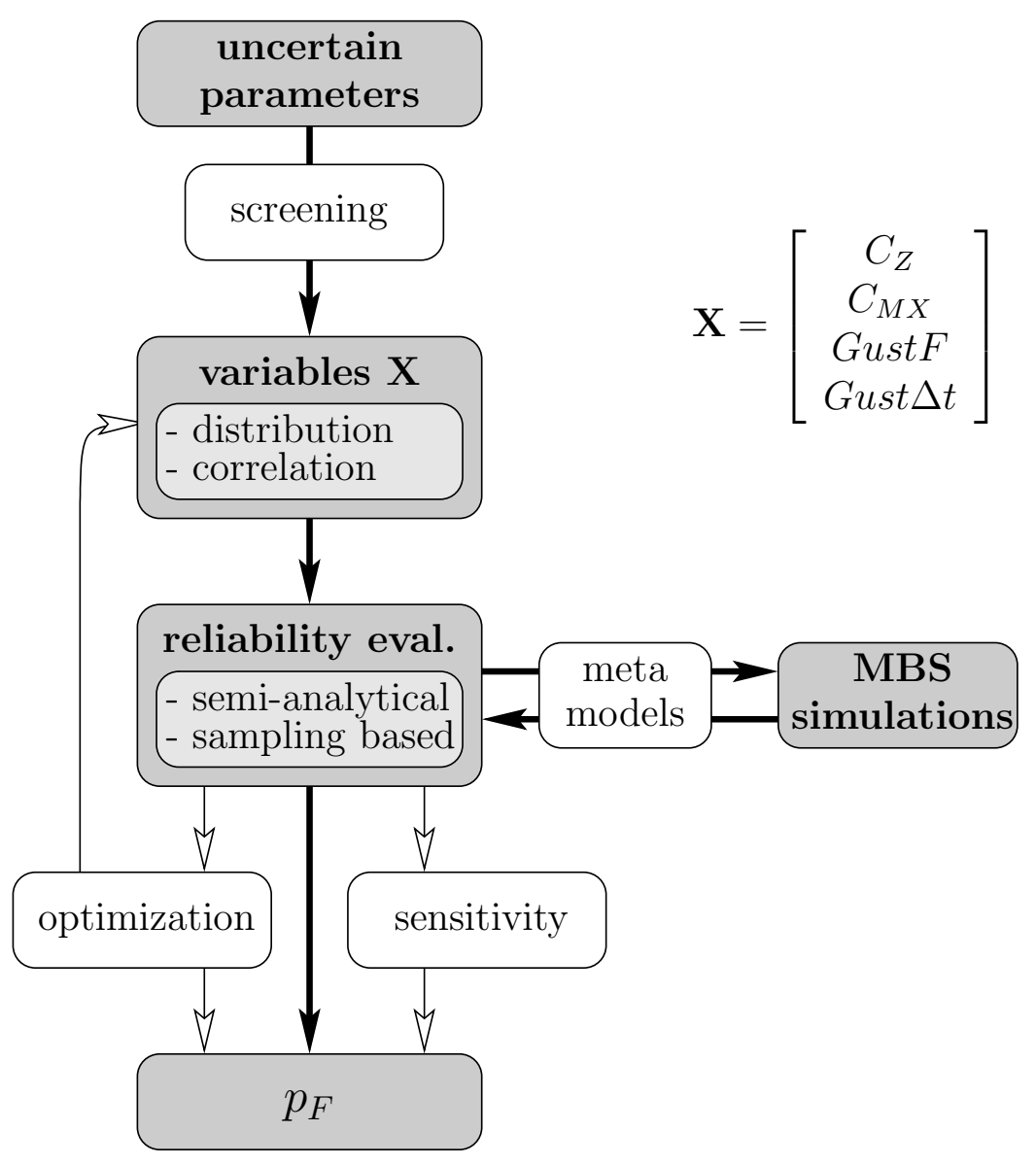

Figure 4.1: Overview of the reliability evaluation process; the gray boxes denote the main workflow.

The reliability evaluation process is summarised in Fig. 4.1. Starting point is the identification of the uncertain parameters which are then modelled as stochastic variables $X_{i}$. These are defined by their probability distributions, whereas their possible statistical interdependency is given by the correlation matrix or even the joint probability distributions. Screening experiments can help to select most influential parameters and thus to reduce the number of variables. The variables considered in this work have been 


\section{Reliability evaluation}

already set in the previous chapter: the aerodynamic lift and roll moment coefficients, the gust factor and the gust duration, i.e. $\mathbf{X}^{T}=\left[C_{Z}, C_{M X}\right.$, Gust Factor, Gust $\left.\Delta t\right]$, the statistics of the variables having been summarised in Table 3.1 (page 44) and Fig. 3.5 (page 50). Correlation could not be defined because of the lack of information; however, in this chapter methods for the analysis of correlated variates will be presented and tested on hypothetical correlation scenarios.

The stochastic variables are passed to the reliability evaluation module which consists of the algorithms for the quantification of the probability of failure $p_{F}$. To this aim different techniques, semi-analytical and sampling based, are used. The reliability evaluation module obviously needs to know the behaviour of the system. In the crosswind stability analysis such behaviour is not described explicitly but only implicitly by means of computational models; in this work, as in most norms, multibody simulation is used. Even though multibody simulations are computationally not very expensive, large number of runs can be necessary; in this case metamodels, which are simpler and less accurate models like response surfaces substituting the original model, can help to reduce the overall effort.

In addition to the simple evaluation of the probability of failure, the sensitivity of the probability of failure with respect to the statistics of the parameters is also studied in this work. Moreover, simple reliability based optimisation loops have been tested. The optimisation module could use information from the sensitivity analysis module but this approach would require dedicated optimisation algorithms; on the contrary, conventional algorithms have been used in this work.

The main components of the framework will be discussed at first; afterwards the additional blocks (screening, metamodels, sensitivity and optimisation) are addressed. In this chapter all the methodologies are exemplified by the straight track case with a driving velocity of $250 \mathrm{~km} / \mathrm{h}$, which will be denoted as test case.

\subsection{Basic concepts}

\subsubsection{Probability of failure}

In this section the basic concept of reliability analysis is summarised and exemplified by a one dimensional example. For the notation, the following convention is used:

uppercase $=$ stochastic variable

lowercase $=$ outcome of the stochastic variable or deterministic parameter.

Consider a generic time-independent system, the behaviour of which is determined by a single parameter $x \in \mathbb{R}$, Fig. 4.2 If the value of $x$ lays in the subset $\Omega_{F}=\{x \in$ $\left.\mathbb{R}: x>x_{L}\right\}$, called failure region, the behaviour of the system does not meet the demand, otherwise it does. In the crosswind stability the system is the driving vehicle including the scenario and many parameters (aerodynamic coefficients etc. as defined above) determine the behaviour of the system. In general, the failure region $\Omega_{F}$ is the subset of all possible parameter configurations $\mathbf{x} \in \mathbb{R}^{n}$ for which the crosswind stability is not ensured, leading for example to a wheel unloading that exceeds a given limit. 


\subsection{Basic concepts}
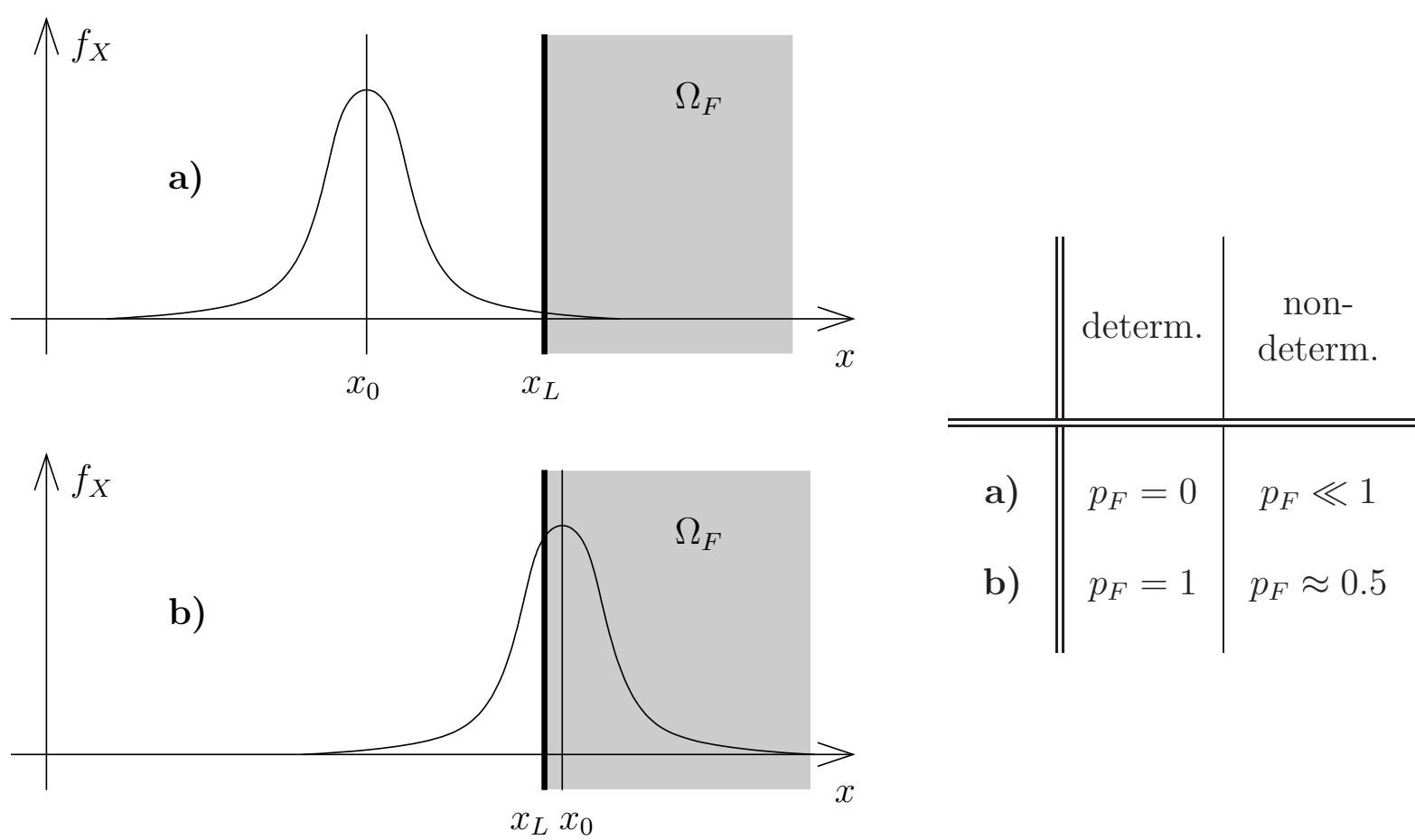

Figure 4.2: Basic concept of reliability analysis in one dimensional case (left) and corresponding probabilities of failure (right).

Referring to Fig. 4.2, in a deterministic approach the parameter $x$ assumes a fixed value $x_{0}$ and thus the system can only be completely safe $\left(x_{0} \notin \Omega_{F}\right.$, i.e. $\left.x_{0} \leq x_{L}\right)$ or completely unsafe $\left(x \in \Omega_{F}\right.$, i.e. $\left.x_{0}>x_{L}\right)$. On the contrary, in a non-deterministic approach uncertainty and variability in the parameter $x$ are considered and $x$ cannot be assigned a single fixed value. The parameter is then modelled as a stochastic variable, $X$, with a probability distribution function $f_{X}$. It is reasonable to assume that the expected value of $X$ is the nominal value $x_{0}$ used in the deterministic approach. The case in which the failure region $\Omega_{F}$ is also nondeterministic, i.e. $x_{L}$ is also a stochastic variable, can be reduced to the case of deterministic failure region considering the difference $x_{L}-x_{0}$.

In the nondeterministic approach the safety of the system can only be assessed statistically, namely by the probability that the system parameter lies in the failure region, which is called probability of failure:

$$
p_{F}=\operatorname{Pr}\left\{x \in \Omega_{F}\right\}=\int_{\Omega_{F}} f_{X}(x) \mathrm{d} x
$$

It necessarily is $p_{F} \in(0,1)$. The deterministic approach can also be assessed by (4.1), setting $f_{X}(x)=\delta\left(x_{0}\right)$; in this way the deterministic safe case corresponds to $p_{F}=0$, whereas the unsafe case to $p_{F}=1$, see the table in Fig. 4.2. The expression (4.1) holds in the same form also for the generic multidimensional case if the scalar $x$ is substituted by a vector $\mathbf{x}$. 


\section{Reliability evaluation}

Assuming that $X$ is an unbounded stochastic variable an outcome $x>x_{L}$ of $X$ is always possible, i.e. the system cannot be completely safe. This fact is often used to claim that the non-deterministic approach is in general conservative. On the other hand, when $x_{0}>x_{L}$, the system is not completely unsafe, as would be in the deterministic case. For example, if $x_{0} \rightarrow x_{L}^{+}$and the PDF of $X$ is symmetric, it is $p_{F} \rightarrow 0.5$, as in Fig. $4.2 \mathrm{~b}$ ). This is to say, the nondeterministic approach offers a more realistic model of real phenomena, provided that sufficient information for the statistical description of the parameters is available.

\subsubsection{Limit state}

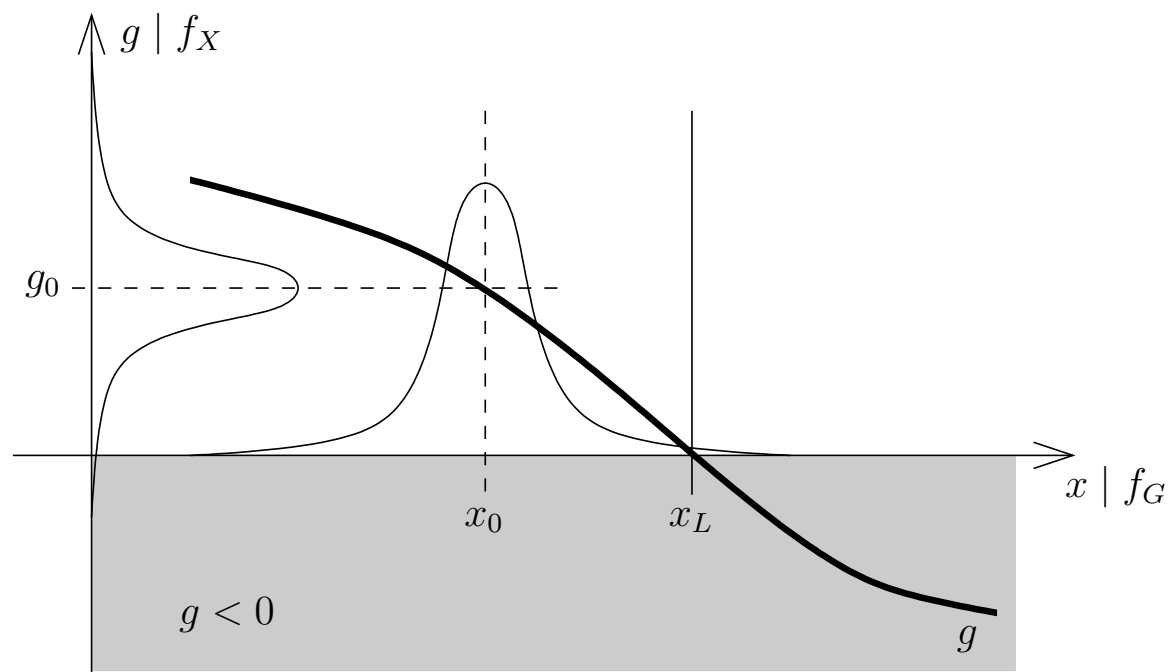

Figure 4.3: Definition of limit state. Note that $x_{0}$ is defined to be equal to $\mu_{X}$ but $g_{0}=g\left(x_{0}\right)$ is in general not necessarily equal to $\mu_{G}$.

As already mentioned, the failure region $\Omega_{F}$ is the subset of the parameter space for which the system behaviour is unsafe. Such behaviour is quantified by the performance function $g(x)$. This function can be always formulated so that $\Omega_{F}$ is:

$$
\Omega_{F}=\{x \in \mathbb{R}: g(x)<0\},
$$

see Fig. 4.3. The equation

$$
g(x)=0,
$$

which defines the boundaries of $\Omega_{F}$ ( $x_{L}$ in the one dimensional example), is called limit state.

In the deterministic approach only the value $g_{0}=g\left(x_{0}\right)$ is considered: if $g_{0}>0$ the system is completely safe, otherwise it is not. But if the stochastic variable $X$ is considered, the performance function defines a new stochastic variable ${ }^{1} G=g(X)$ with

\footnotetext{
${ }^{1}$ An outcome of $G$ will be denoted as $g$; the performance function $g$ will be denoted as $g(x)$ where ambiguity can arise.
} 


\subsection{Basic concepts}

statistics depending on the statistics of $X$ and on the relation $g(x)$. In analogy with (4.1), the probability of failure can be then defined with respect to $G$ as:

$$
p_{F}=\operatorname{Pr}\{G<0\}=\int_{g(x)<0} f_{G}(g) \mathrm{d} g .
$$

The advantages and the necessity for the two formulations (4.1) and (4.4) for the evaluation of $p_{F}$ will be discussed in the next sections.

Two points must be stressed:

Implicit limit state For a given problem the performance function $g(x)$ is seldom known analytically. More often, and this is also the case of the crosswind stability, it is defined by a simulation code which maps $x$ into $g$. It follows that the limit state (4.3) cannot be expressed in an explicit form, but only in an implicit one. The main consequence is that the failure domain $\Omega_{F}$ is unknown and neither (4.1) nor (4.4) can be directly used to compute the probability of failure because the domain of integration is not known ${ }^{2}$. This fact largely influences the methodologies for the reliability evaluation.

Multiple limit states In general it is possible to have more than one limit state. The resulting overall failure region is the union of the failure regions defined by the single limit states. Many limit states can arise also in the crosswind stability if different criteria are checked (overturning, derailment, track shift). The handling of multiple limit states does not change the formulation of the problem but can influence some of the computational methods for the reliability analysis presented in the next sections. However, as the effects due to overturning are predominant, only the limit state arising from this criterion is considered in this work.

\subsubsection{Limit state for the crosswind stability}

In the crosswind stability task the performance function is related to the wheel unloading which is a function of the outcome of the variables $\mathbf{X}$, the mean wind velocity $U_{0}$ and the driving velocity $V$, i.e. $Q^{\prime}=Q^{\prime}\left(\mathbf{x} ; V, U_{0}\right)$. As already discussed in Sec. 2.1.3, the reliability analysis copes only with the variables $\mathbf{X}$, because in the risk analysis the mean wind velocity and the driving velocity are deterministic parameters.

However, the performance function $g(x)$ is not identical to the wheel unloading $Q^{\prime}$ because failure has to arise for $g(x)<0$ whereas $Q^{\prime}>1$ in the case of failure. Thus, if the limit for the unloading is $Q_{L}^{\prime}$ the performance function has to be defined as:

$$
g\left(\mathbf{x} ; Q_{L}^{\prime}, V, U_{0}\right)=Q_{L}^{\prime}-Q^{\prime}\left(\mathbf{x} ; V, U_{0}\right) .
$$

A widely used value for $Q_{L}^{\prime}$ is 0.9 , corresponding to a safety limit of $10 \%$. On the contrary, $Q_{L}^{\prime}=1$ is used in this work because, according to the probabilistic approach,

\footnotetext{
${ }^{2}$ In the one dimensional case used as example the value of $x_{L}$ could be easily determined by numerical methods, but this is not true for a generic multidimensional case.
} 


\section{Reliability evaluation}

the safety limit is substituted by the probability of failure. The underlying assumption is that the variables $\mathbf{X}$ cover all the uncertainties; in practice a little safety limit should be still considered, as the variables $\mathbf{X}$ represent only a part of all uncertainties.

\subsection{Evaluation of the probability of failure}

In this section only the most basic methods for the evaluation of the probability of failure are reported. However, they are also the most widely used because it is not reasonable to strive for high accuracy on the computational side when only poor statistical information is available for the parameters. As large literature exists, e.g. [MKL86, DM96, Mel99], only the most basic definitions, the final formulas and comments for the specific task of the crosswind stability are reported in what follows.

\subsubsection{Semi-analytical techniques}

The most common indicator for the safety of a system is the reliability index:

$$
\beta=\frac{\mu_{G}}{\sigma_{G}}
$$

which gives the distance, in standard deviation units, of the expected value of the performance function $G=g(X)$ from the failure region $g(x)<0$, see Fig. 4.4.

If $G$ is gaussian, e.g. when $X$ is gaussian and the relation $g(x)$ is linear, the probability of failure is:

$$
p_{F}=1-\Phi(\beta)=\Phi(-\beta)
$$

$\Phi$ being the gaussian CDF.

The question is how to compute $\mu_{G}$ and $\sigma_{G}$ in the general multidimensional case of implicit limit state and then how to use $\beta$ to estimate the probability of failure in the general case of nongaussian $G$.

\section{Mean value}

In the Mean Value (MV) approximation the performance function $g(x)$ is linearised at the mean value of the parameter $\mu_{X} \equiv x_{0}$, see Fig. 4.4. For a generic multidimensional case the statistics are:

$$
\begin{aligned}
\mu_{G} & =g\left(\boldsymbol{\mu}_{\mathbf{X}}\right) \\
\sigma_{G} & =\sqrt{\left|\sum_{i=1}^{n} \sum_{j=1}^{n} \frac{\partial g}{\partial x_{i}} \frac{\partial g}{\partial x_{j}}\right|_{\mathbf{x}=\boldsymbol{\mu}_{\mathbf{x}}} \operatorname{Cov}\left(X_{i}, X_{j}\right)},
\end{aligned}
$$

$\operatorname{Cov}\left(X_{i}, X_{j}\right)$ being the $(i, j)$ element of the covariance matrix. If the parameters are uncorrelated $(4.8 \mathrm{~b})$ reduces to:

$$
\sigma_{G}=\sqrt{\sum_{i=1}^{n}\left(\left.\frac{\partial g}{\partial x_{i}}\right|_{\mathbf{x}=\mu_{\mathbf{x}}}\right)^{2} \sigma_{X_{i}}^{2}} .
$$




\subsection{Evaluation of the probability of failure}

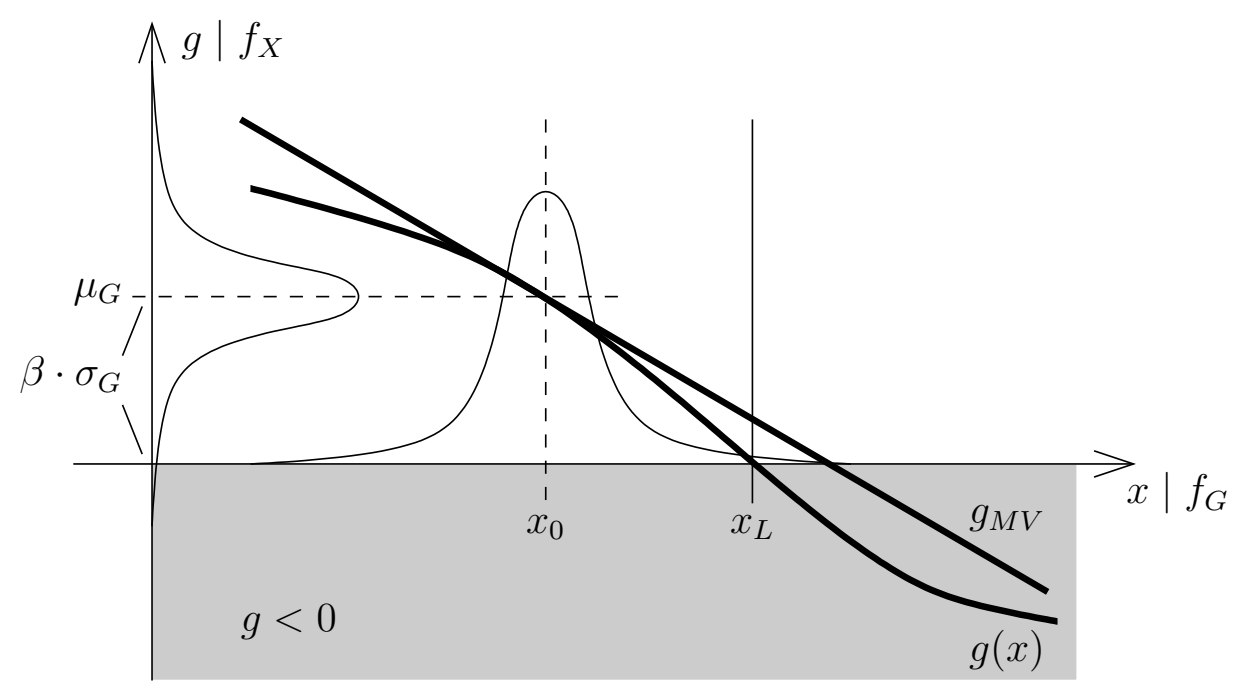

Figure 4.4: Mean value approximation. $g_{M V}$ is the performance function $g(x)$ linearised at $x_{0}$.

It follows that the reliability index is:

$$
\beta_{M V}=\left.\frac{\mu_{G}}{\sigma_{G}}\right|_{g_{M V}}
$$

having stressed that $\mu_{G}$ and $\sigma_{G}$ are computed using the linearised performance function $g_{M V}(x)$. The probability of failure is then evaluated by (4.7), which is equivalent to impose to $G$ a normal distribution with mean $\mu_{G}$ and standard deviation $\sigma_{G}$.

It muss be stressed that the Mean Value method is based on a poor approximation. Firstly, the probability distributions of the parameters do not play any role but only their first and second order moments. Moreover, $g(x)$ is approximated very poorly in the region $g(x)<0$ which actually determines the probability of failure. Finally, the approximated statistics in (4.8), and thus the computed probability of failure, does not depend on the actual failure region $\left(x_{L}\right.$ in the one dimensional example) but only on the value and the first derivatives of the performance function at the mean value of the parameters.

On the other hand, the Mean Value approximation allows an estimation of the probability of failure with minimal computational effort. For this reason it is the first choice when the performance function $g(x)$ is defined by computationally expensive simulation codes, like FEM.

\section{First order reliability method}

The most common method for reliability analysis is the so called First Order Reliability Method, FORM ${ }^{3}$. This method is based on the fact that, for normal uncorrelated variates

\footnotetext{
${ }^{3}$ This term is actually not correct but commonly used in practice. For a discussion on terminology see for example [HM95].
} 


\section{Reliability evaluation}

$\mathbf{X}$ with zero mean and unit variance and linear performance function $g(\mathbf{x})$, the reliability index $\beta$ is equivalent to the distance of the limit state to the origin of the $\mathbf{x}$ space. In the one dimensional example, if $x$ were gaussian and $g(x)$ linear, it would result $\beta=x_{L}-x_{0}$, see Fig. 4.5.

In the general case of nonnormal correlated variables $X_{i}$ reduced variables $\mathbf{X}^{\prime}$, which are normal uncorrelated with zero mean and unit variance, are introduced:

$$
\mathbf{X}^{\prime}=T(\mathbf{X})
$$

where $T$ is an adequate transformation. If the original variates $\mathbf{X}$ are already gaussian and uncorrelated, the transformation reduces to:

$$
X_{i}^{\prime}=\frac{X_{i}-\mu_{X_{i}}}{\sigma_{X_{i}}}
$$

the problem of correlated and nonnormal variables will be addressed later. The performance function is correspondingly reformulated in the new coordinates:

$$
g^{\prime}\left(x_{1}^{\prime}, x_{2}^{\prime}, \ldots, x_{n}^{\prime}\right)=g\left(x_{1}, x_{2}, \ldots, x_{n}\right),
$$

and the reliability index is evaluated as the minimal distance of the (in general nonlinear) limit state $g^{\prime}\left(\mathbf{x}^{\prime}\right)$ to the origin of the transformed space. The point $\mathbf{x}^{\prime *}$ on the limit state of minimal distance to the origin is called Most Probable (failure) Point, MPP. Finally, the probability of failure is evaluated as $\Phi(-\beta)$, with the same assumptions made for the Mean Value approach.

Interesting is the interpretation of the FORM method given by [AT84]. The reliability index computed by FORM is shown to be equivalent to the ratio $\mu_{G} / \sigma_{G}$ when the limit state linearised at the MPP, $g_{F O R M}$, is used instead of the original limit state $g$ :

$$
\beta_{F O R M}=\left.\frac{\mu_{G}}{\sigma_{G}}\right|_{g_{F O R M}} .
$$

This is illustrated for the one dimensional case, where the limit state degenerates to a point, in Fig. 4.5.

The main question is how to determine the MPP. In general the task can be posed as an optimisation problem; as the performance function is only known implicitly, it has to be formulated in a very general way:

$$
\begin{gathered}
\min _{\mathbf{x}^{\prime}}\left(\mathbf{x}^{\prime T} \mathbf{x}^{\prime}\right)^{1 / 2} \\
g^{\prime}\left(\mathbf{x}^{\prime}\right)=0
\end{gathered}
$$

The starting search point is usually chosen as the mean value $\boldsymbol{\mu}_{\mathbf{x}^{\prime}}$, leading to an infeasible optimisation problem.

Even though the optimisation approach is very general, it can be computational expensive; special algorithms for the MPP search have thus been developed. In this work the algorithm of Rackwitz and Fiessler was used, see for example [HM95]. Even if 


\subsection{Evaluation of the probability of failure}

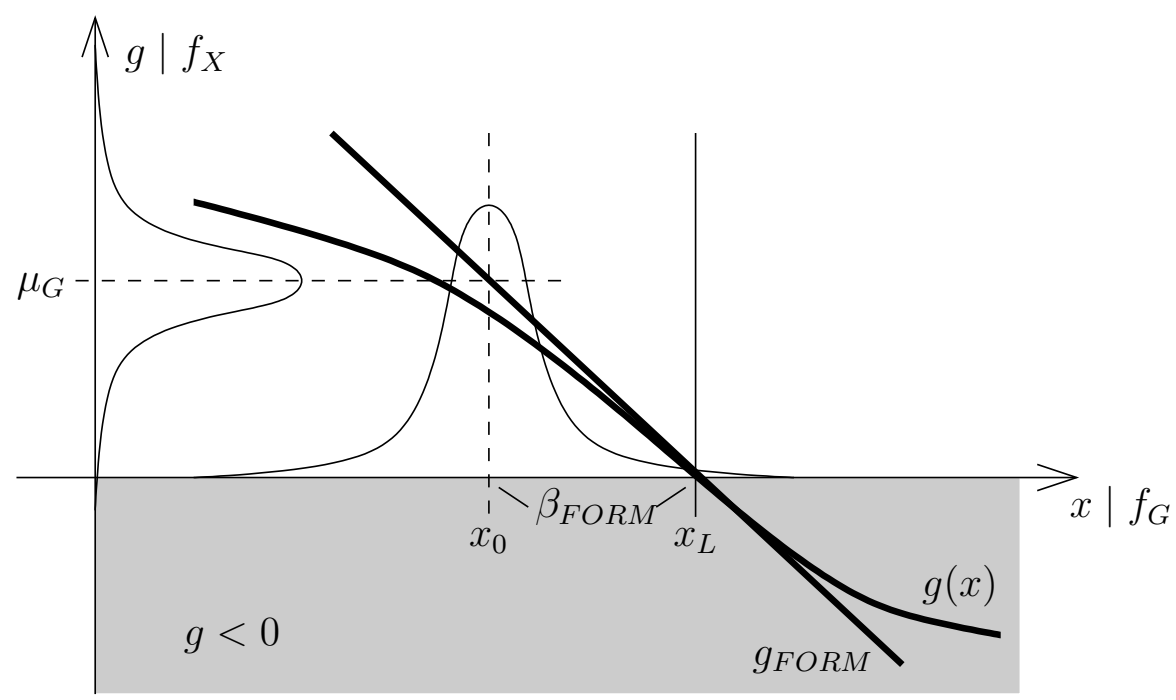

Figure 4.5: FORM approach. $g_{F O R M}$ is the performance function $g(x)$ linearised at the most probable point, which is equivalent, in the one dimensional case, to the degenerated limit state $x_{L}$.

convergence is usually reached within 10 iterations, the number of evaluations of $g^{\prime}(\mathbf{x})$ is relatively large, because in every iteration one evaluation and the computation of the gradient of $g^{\prime}$ is necessary. Thus, as the gradient has to computed by numerical differentiation, at least $2 n+1$ evaluations of $g^{\prime}$ are needed in every iteration, $n$ being the dimension of $\mathbf{X}$.

When the MPP $\left(\mathbf{x}^{\prime *}\right)$ has been found, the reliability index can be expressed as:

$$
\beta_{\text {FORM }}=\sqrt{\sum_{i=1}^{n}\left(x_{i}^{\prime *}\right)^{2}}=\left.\frac{-\sum_{i=1}^{n} x_{i}^{\prime} \frac{\partial g}{\partial x_{i}^{\prime}}}{\sqrt{\sum_{i=1}^{n}\left(\frac{\partial g}{\partial x_{i}^{\prime}}\right)^{2}}}\right|_{\mathbf{x}^{\prime}=\mathbf{x}^{\prime *}},
$$

and the coordinates of the MPP in the transformed space as:

$$
x_{i}^{\prime *}=-\alpha_{i} \beta_{F O R M},
$$

where $\alpha_{i}$ are the direction cosines:

$$
\alpha_{i}=\left.\frac{\frac{\partial g}{\partial x_{i}^{\prime}}}{\sqrt{\sum_{i=1}^{n}\left(\frac{\partial g}{\partial x_{i}^{\prime}}\right)^{2}}}\right|_{\mathbf{x}^{\prime}=\mathbf{x}^{\prime *}}
$$




\section{Reliability evaluation}

Eq. (4.17) shows that the position of the MPP, i.e. the coordinates of $\mathrm{x}^{\prime *}$ or equivalently the direction cosines, give basic information about the contribution of each parameters to the reliability index, and thus to the probability of failure. The direction cosines will be considered again in the context of sensitivity analysis in Sec. 4.3.
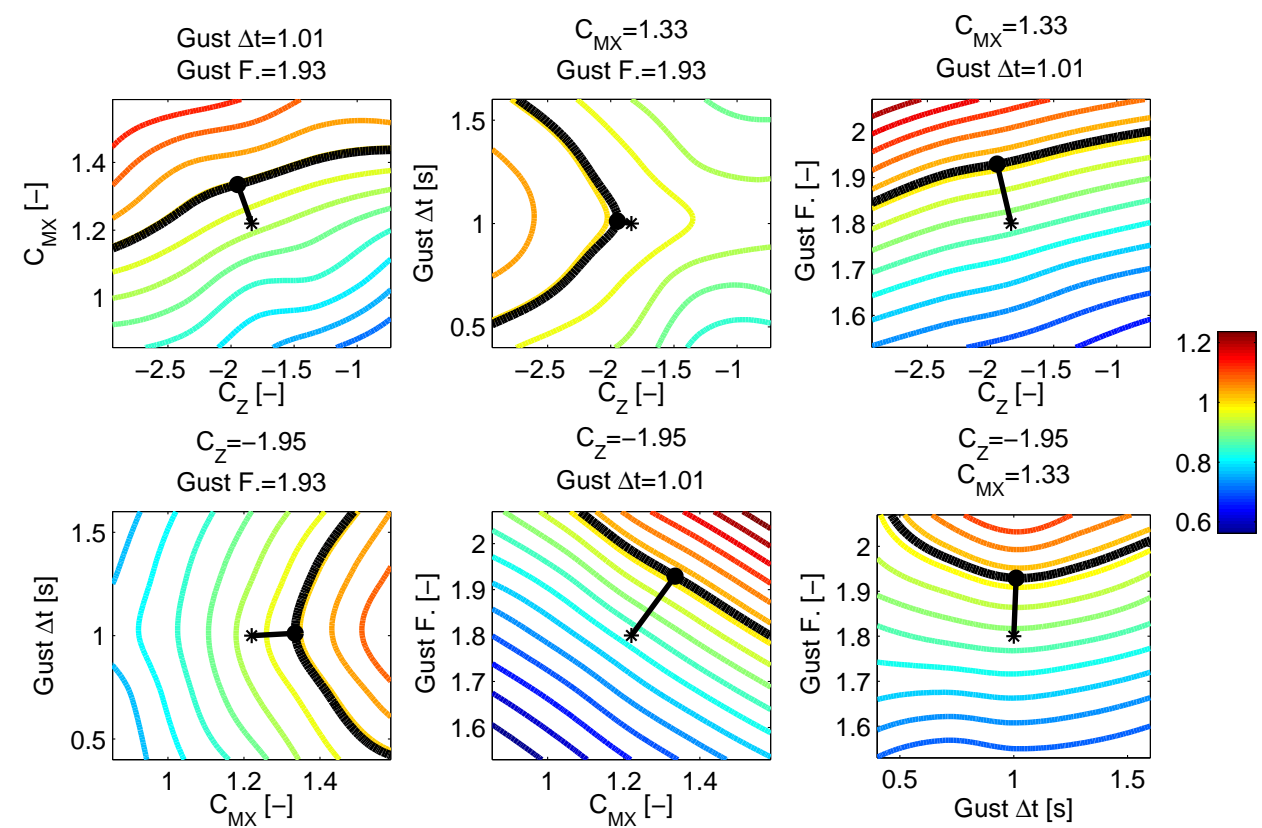

Figure 4.6: Most Proable Point with contours of the performance function $g(x)=Q^{\prime}(x)$ for $U_{0}=16 \mathrm{~m} / \mathrm{s}$. The thick line is the limit state $g(x)=0$, i.e. $Q^{\prime}=1$. Note that the plotted sections are orthogonal planes containing the MPP and not the origin (mean value of the parameters). Thus the plotted origin (asterisk) is a projection of the real origin on the plane.

A drawback of the FORM approach is that in its basic formulation, similarly to the Mean Value approach, the probability distributions of the parameters $\mathbf{X}$ are not considered but only the first and second moments; that is to say, normal distributions with the same mean and variance of the real distributions are implied because for normal variates the FORM approach is exact. On the other hand, statistical information exceeding mean and value is seldom available and thus the approach is a good balance between accuracy and disposable information. A significant improvement with respect to the Mean Value method is to find in the approximation of the performance function: $g(x)$ is namely linearised in the range that actually determines the probability of failure, i.e. at the limit state.

Fig. 4.6 shows the Most Probable Point and its distance from the origin (mean value of the parameters) for the test case at a wind velocity $U_{0}=16 \mathrm{~m} / \mathrm{s}$. The contours and the limit state could be plotted because the MPP search was performed not directly on the performance function but on a response surface. The contours are in general useful to check qualitatively that the search algorithm is not fallen in a local minimum and that the linearisation on which the FORM is based is an acceptable approximation of the performance function. In the case of Fig. 4.6 the presence of nonlinearities is evident but 


\subsection{Evaluation of the probability of failure}

in the whole the FORM can be still expected to give acceptable results; a comparison with other reliability evaluation methods is reported in Sec. 4.2.5.

\section{MPP locus}

Variation of $\boldsymbol{U}_{\mathbf{0}}$ According to the discussion in Sec. 2.1.3, the task of the reliability analysis is to compute the probability of failure parameterised with the driving velocity $V$ and the mean wind velocity $U_{0}$, which influence the probability of failure by influencing the performance function $Q^{\prime}\left(\mathbf{x} ; U_{0}, V\right)$. If FORM is used, the variation of the position of the Most Probable Point when $U_{0}$ and $V$ vary can be tracked. The resulting locus offers an insight in the behaviour of the system, because in the FORM approximation the coordinates of the MPP are proportional to the contribution of each parameter to the probability of failure ${ }^{4}$. In this section only the variation of $U_{0}$ is considered.
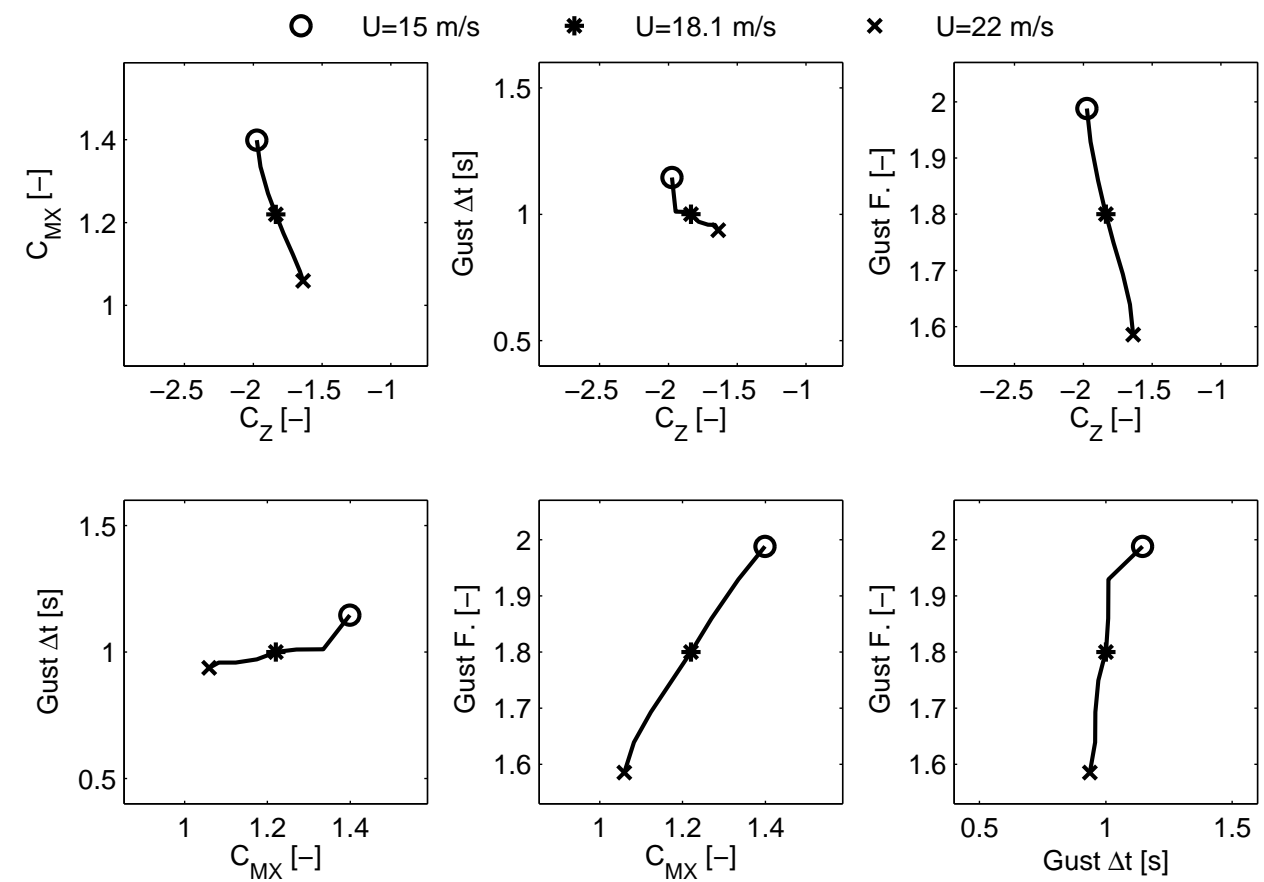

Figure 4.7: MPP locus for $U_{0}$. See also Fig. 4.8.

In Fig. 4.7 the MPP locus for $U_{0}$ in the range $13 \div 20 \mathrm{~m} / \mathrm{s}$ is plotted. It can be well observed that the behaviour of the system is almost linear and the contributions to the probability of failure of the gust length and the lift coefficient are comparatively little. The corresponding probability of failure is reported in Fig. 4.8 and is compared with the Mean Value approximation. For low values of $p_{F}$, which corresponds to the lower mean wind velocities and are actually the most sensitive and important, the agreement with the results computed by FORM is acceptable.

\footnotetext{
${ }^{4}$ This is true in the space of the reduced variables $\mathbf{X}^{\prime}$. In what follows the MPP loci are plotted in the space of the original variables $\mathbf{X}$ and thus the coordinates of the MPP must be considered relatively to $\boldsymbol{\mu}_{\mathbf{X}}$, i.e. to the centre point of the plots.
} 


\section{Reliability evaluation}

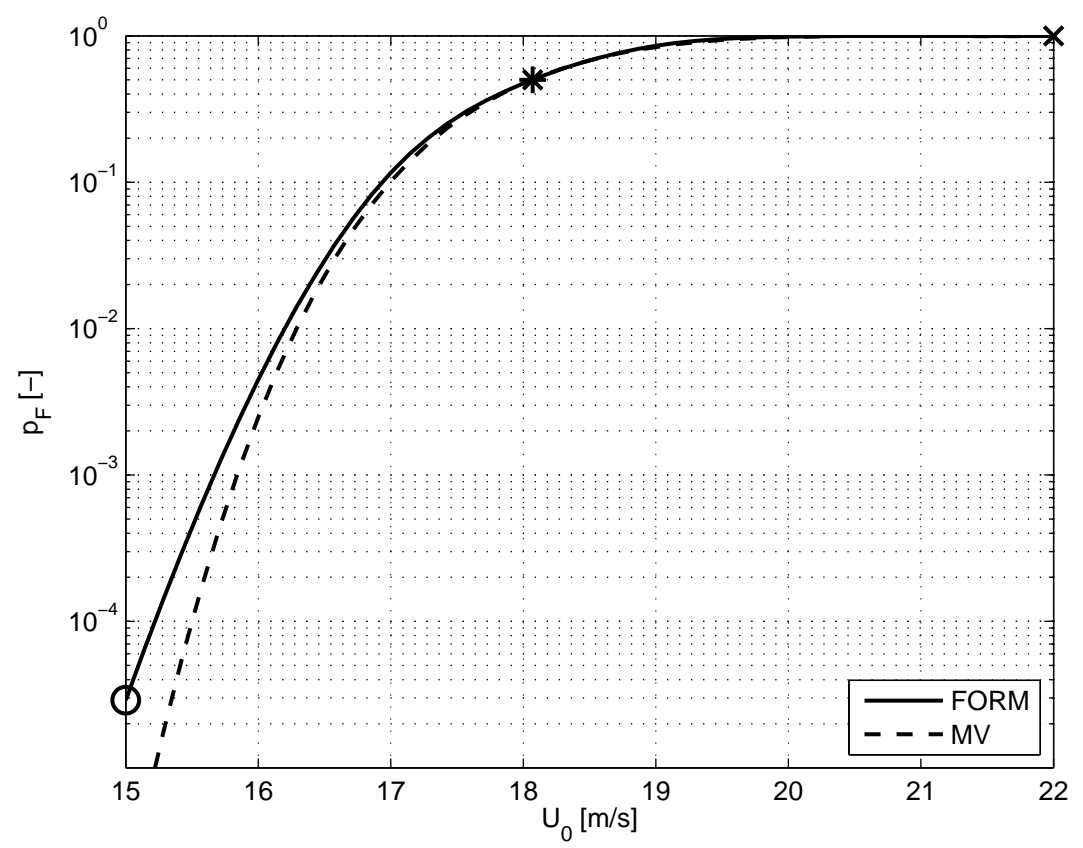

Figure 4.8: $p_{F}$ as a function of $U_{0}$, corresponding to Fig. 4.7.

Variation of $\mathrm{Q}_{\mathrm{L}}^{\prime} \quad$ It is also interesting to track the position of the MPP and to compute the resulting probability of failure for a variation of the wheel unloading limit. It was shown that the limit state $g=0$ for the crosswind stability task can be expressed as:

$$
Q^{\prime}\left(\mathbf{x} ; U_{0}, V\right)=Q_{L}^{\prime}
$$

$Q_{L}^{\prime}$ being the limit value for the wheel unloading. It follows that the limit state changes when $Q_{L}^{\prime}$ changes, without any modification to the proper performance function $Q^{\prime}\left(\mathbf{x} ; U_{0}, V\right)$. The results are not directly needed for the risk analysis, in which a fixed limit for the wheel unloading is considered; the choice of a value for $Q_{L}^{\prime}$ is actually a task of the safety management and is based on considerations on the driving dynamics as well as experience. The aim of the locus is to facilitate this choice by provision of additional information, e.g. the variation of the probability of failure for a given variation of $Q_{L}^{\prime}$.

Fig. 4.9 shows the MPP locus for $Q_{L}^{\prime}$ in the range $0.85 \div 1.1$ for $U_{0}=16 \mathrm{~m} / \mathrm{s}$; the meaning of a wheel unloading larger than unity was discussed in Sec. 3.1.1. It can be observed again that the behaviour of the system is almost linear. This means that the choice of a value for $Q_{L}^{\prime}$ can be performed without considering the uncertainties in the parameters, because their relative contributions to the probability of failure are the same for different values of $Q_{L}^{\prime}$. The corresponding change in the probability of failure is reported in Fig. 4.10: the curve quantifies the probability of failure connected with different safety margins $\left(1-Q_{L}^{\prime}\right)$ and allows a founded choice of a safety limit. Even though the curve refers to a single mean wind velocity, the same trend is observed for other velocities. 


\subsection{Evaluation of the probability of failure}
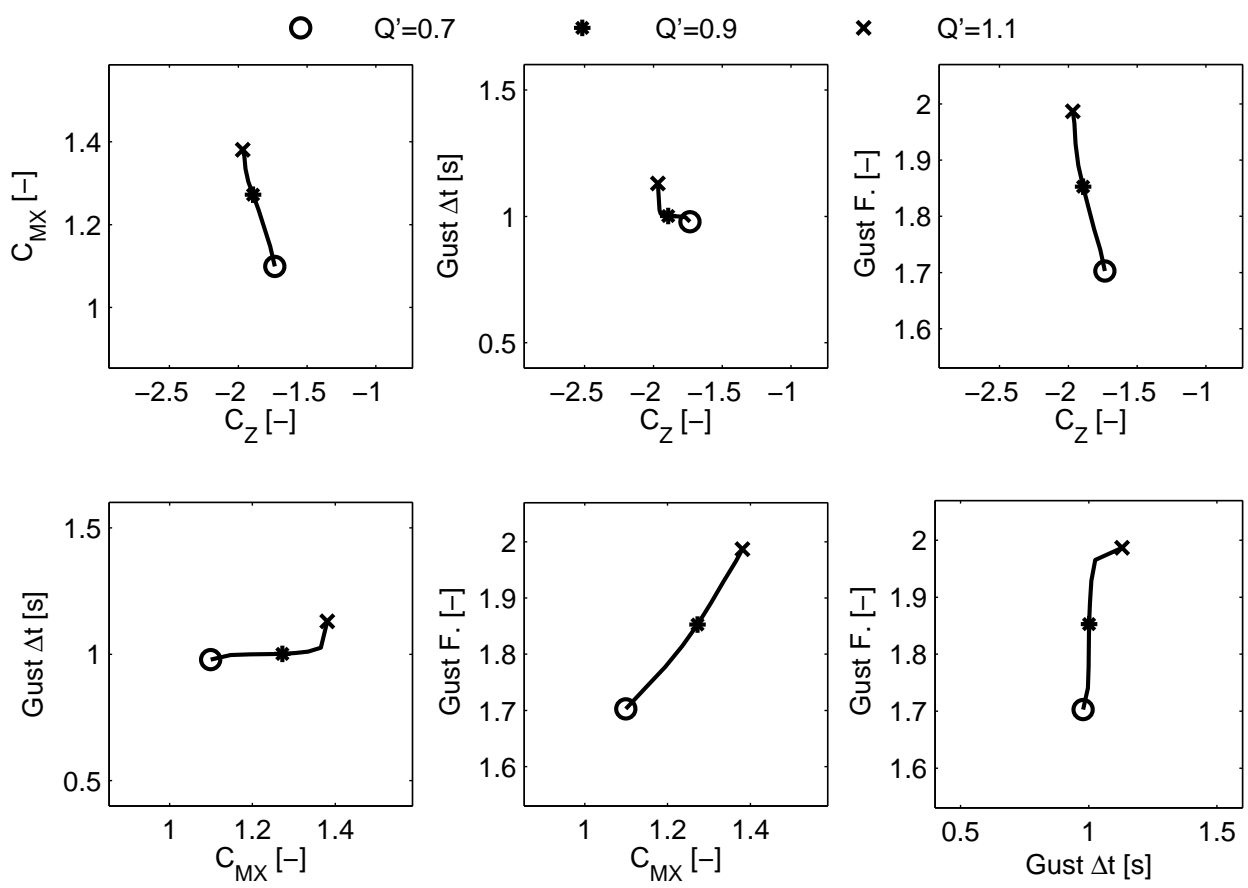

Figure 4.9: MPP locus for $Q_{L}^{\prime} \in[0.7 ; 1.1]$ and $U_{0}=16 \mathrm{~m} / \mathrm{s}$. See also Fig. 4.10.

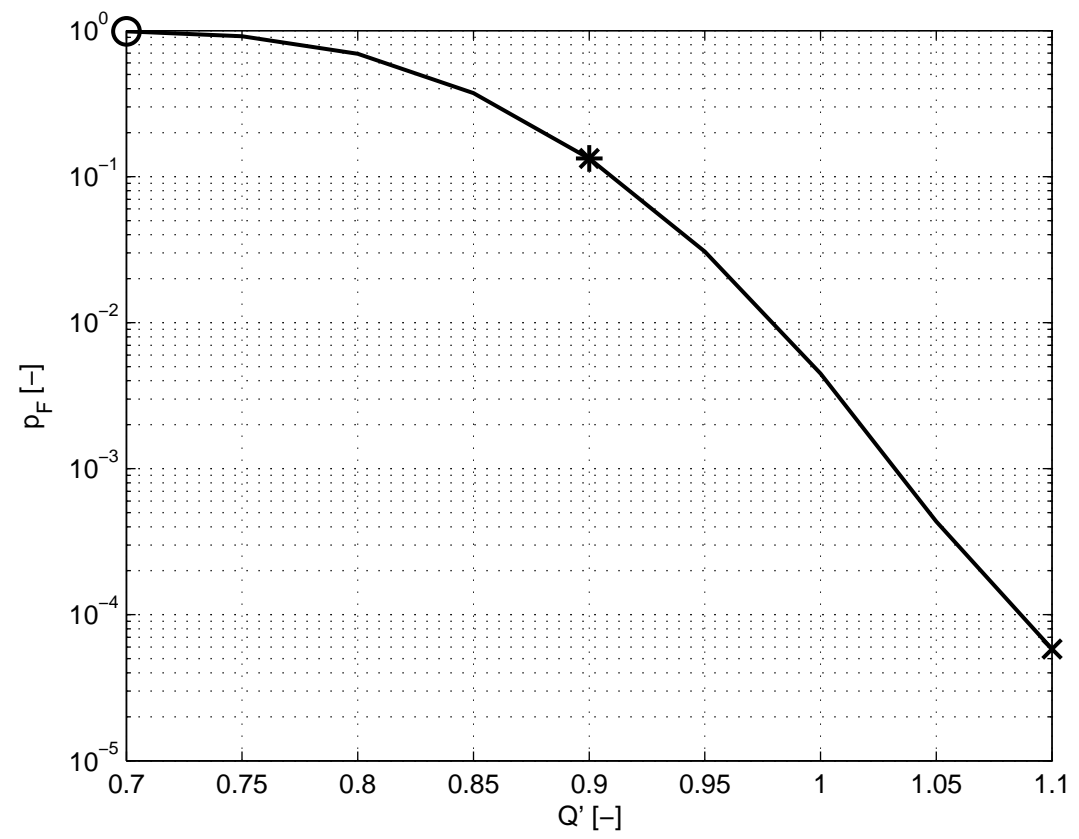

Figure 4.10: $p_{F}$ as a function of $Q_{L}^{\prime}$ for $U_{0}=16 \mathrm{~m} / \mathrm{s}$, corresponding to Fig. 4.9 . 


\section{Reliability evaluation}

\subsubsection{Nonnormal and correlated variates}

In the previous section it was assumed that the variables $\mathbf{X}$ are normal and uncorrelated. In this section methods for the handling of nonnormal and/or correlated variates are reported.

\section{Rosenblatt transformation}

The Rosenblatt transformation maps a set of correlated random variables into a set of independent gaussian ones, offering the solution to both problems of nonnormality and correlation:

$$
x_{i}^{\prime}=\Phi^{-1}\left(F_{X_{i}}\left(x_{i} \mid x_{1}, \ldots, x_{i-1}\right)\right),
$$

where $\Phi^{-1}$ is the inverted normal CDF and $F_{X_{i}}\left(x_{i} \mid x_{1}, \ldots, x_{i-1}\right)$ the CDF of the $t$-th variable conditional to $x_{1}, \ldots, x_{i-1}$. For uncorrelated variables the transformation reduces to

$$
x_{i}^{\prime}=\Phi^{-1}\left(F_{X_{i}}\left(x_{i}\right)\right),
$$

$F_{X_{i}}\left(x_{i}\right)$ being the marginal distribution of $X_{i}$; it can be applied separately to all the elements of $\mathbf{X}$ and is sometimes referred to as marginal transformation. In Fig. 4.11 the transformation (4.21) is plotted for two bounded distributions; it can be clearly seen that the transformation is almost linear in proximity of the mean value, whereas it becomes strongly nonlinear near the bounds.

On the contrary, for correlated variables the general relation (4.20) must be used. To this aim the conditional CDF $F_{X_{i}}\left(x_{i} \mid x_{1}, \ldots, x_{i-1}\right)$ is needed and can be computed from the joint PDF of the variables. This means that for correlated variates, complete statistical information is necessary. An approximated solution for the case of correlated variates when only the covariance matrix is known as well as other possible transformations are reported for example in [DM96] and will not be presented in this work.

For the estimation of $p_{F}$ the Rosenblatt transformation can be applied in two ways. It can be either used to reformulate the limit state in the space of transformed normal variates; the MPP search is then performed in this space. Alternatively the transformation can be embedded in the FORM approach. In practice the former procedure is seldom practicable because the limit state is only given in an implicit form. However, when a metamodel like response surface is used the limit state can be computed numerically. In Fig. 4.12 the effect of the Rosenblatt transformation on the limit state is reported for the test case, having assumed triangular distributions for gust factor and gust length. As the triangular distribution is bounded, the transformation is not defined over the whole parameter space.

\section{Rackwitz-Fiessler (R-F)}

The R-F formula determines an equivalent normal distribution for nonnormal uncorrelated variates by imposing that the original and the equivalent normal distributions have the same value of CDF and PDF at the Most Probable Point $\mathbf{x}^{*}$ (two-parameter 


\subsection{Evaluation of the probability of failure}

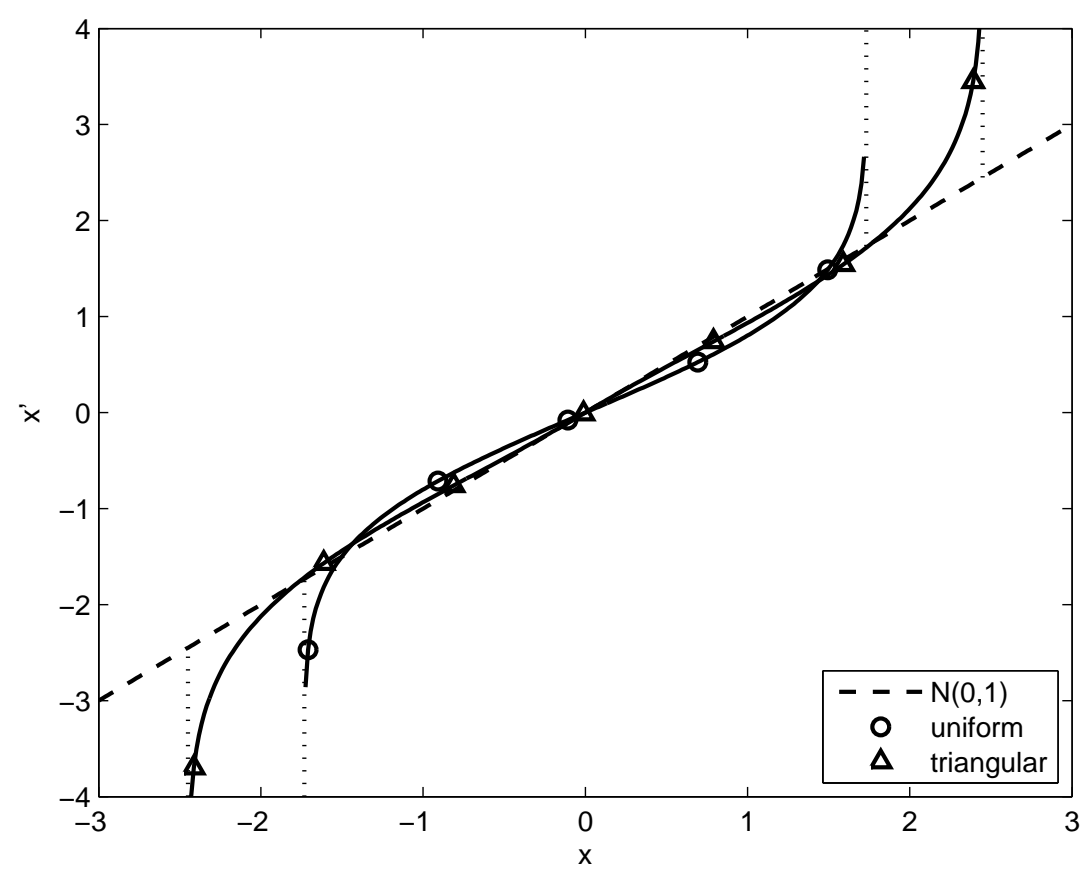

Figure 4.11: Rosenblatt transformation (4.21) for uniform and triangular distributions $(\mu=0$, $\sigma=1)$.

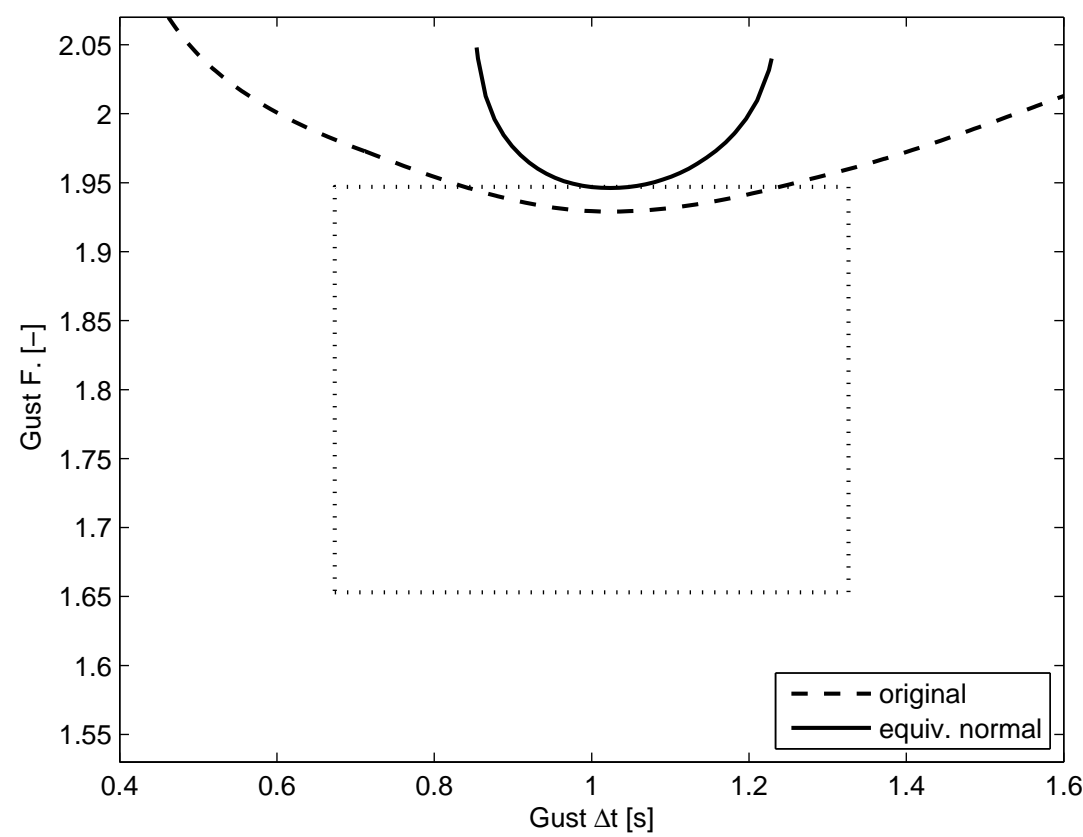

Figure 4.12: Example of Rosenblatt transformation for variables with triangular distribution. The dashed line is the original limit state, the continuous line is the limit state corresponding to the equivalent normal variables. The triangular PDF is $>0$ only in the region delimited by the dotted square; therefore the Rosenbaltt transformation is not defined outside the square. 


\section{Reliability evaluation}

approximation). For the generic $i$-th variable, with $\operatorname{PDF} f_{i}$ and $\mathrm{CDF} F_{i}$, the mean and the standard deviation of the equivalent distribution are:

$$
\begin{aligned}
\left(\mu_{R F}\right)_{i} & =x_{i}^{*}-\sigma_{R F} \Phi^{-1}\left[F_{i}\left(x_{i}^{*}\right)\right] \\
\left(\sigma_{R F}\right)_{i} & =\frac{\phi\left\{\Phi^{-1}\left[F_{i}\left(x_{i}^{*}\right)\right]\right\}}{f_{i}\left(x_{i}^{*}\right)}
\end{aligned}
$$

$\phi$ and $\Phi$ being respectively the normal PDF and CDF. An exemplary plot of the R-F formula is reported in Fig. 4.13, having assumed that the gust factor has a triangular distribution with same variance of the original distribution. As the R-F formula is actually not a transformation but a punctual approximation, the original reliability problem cannot be just reformulated in the new space. The formula must compulsory be integrated in the MPP search algorithm.
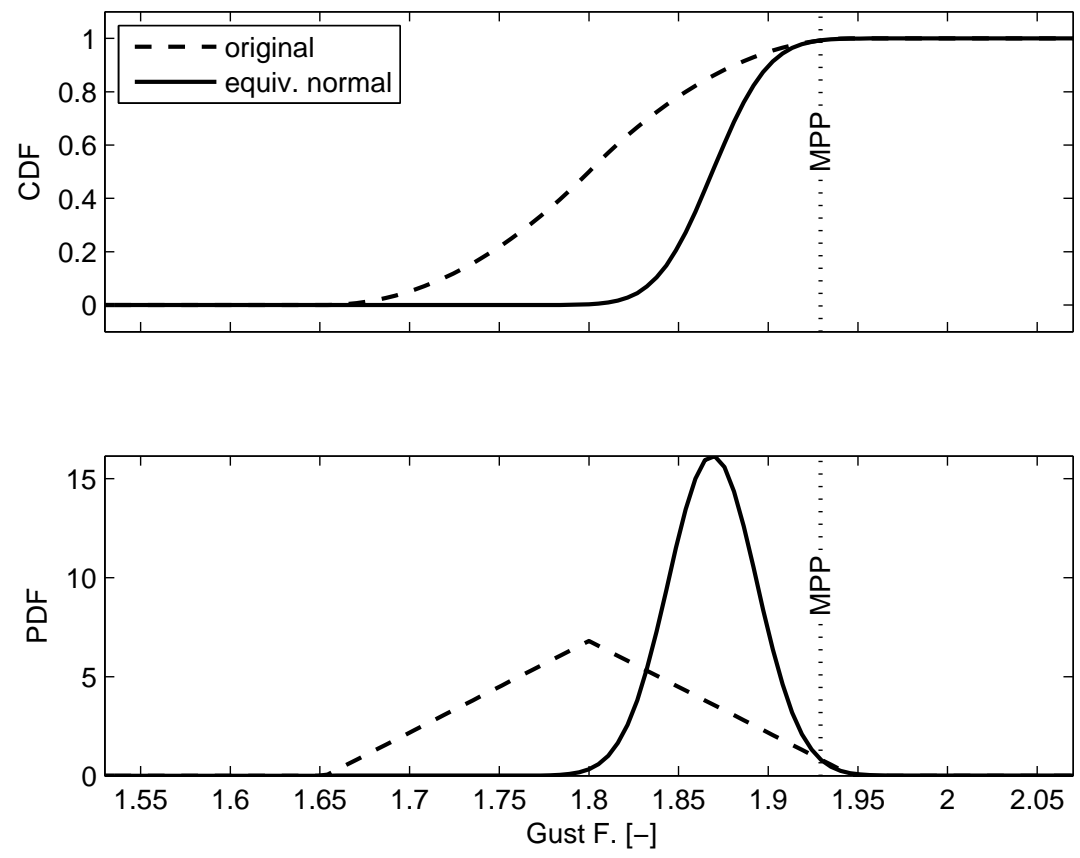

Figure 4.13: Example of R-F method. At the value of the parameter corresponding to the MPP the original triangular (dashed line) and the equivalent normal (continuous line) distribution have the same value of cumulative and probability density function.

\section{Orthogonal transformation}

It has been discussed above that the exact solution offered by the Rosenblatt transformation for correlated variables cannot be applied in practice because of the ignorance of the joint PDF. However, uncorrelated variates $\mathbf{Y}$ can also be obtained by a linear orthogonal transformation. 


\subsection{Evaluation of the probability of failure}

For this purpose consider normal correlated variables and the corresponding reduced variables $\mathbf{X}^{\prime}$, see (4.12). The covariance matrix of the reduced variables is equivalent to the correlation matrix of the original variates $\mathbf{X}$ :

$$
\mathbf{C}^{\prime}=\left[\begin{array}{cccc}
1 & \rho_{12} & \ldots & \rho_{1 n} \\
& 1 & \ldots & \rho_{2 n} \\
& & \ddots & \vdots \\
\text { sym. } & & & 1
\end{array}\right]
$$

where $\rho$ denotes the correlation coefficients. Uncorrelated variates are obtained if a transformation is chosen in the form:

$$
\mathbf{Y}=\mathbf{T}^{T} \mathbf{X}^{\prime}
$$

$\mathbf{T}$ being an orthogonal matrix. This is achieved if $\mathbf{T}$ is composed of the eigenvectors of the correlation matrix $\mathbf{C}^{\prime}$ :

$$
\mathbf{T}^{T} \mathbf{C}^{\prime} \mathbf{T}=[\lambda]
$$

with $[\lambda]$ diagonal matrix of the eigenvalues of $\mathbf{C}^{\prime}$. It is now sufficient to observe that the eigenvalues matrix $[\lambda]$ is also the covariance matrix $\mathbf{C}_{\mathbf{Y}}$ of the transformed variates $\mathbf{Y}$ :

$$
\mathbf{C}_{\mathbf{Y}}=E\left[\mathbf{Y} \mathbf{Y}^{T}\right]=E\left[\mathbf{T}^{T} \mathbf{X}^{\prime} \mathbf{X}^{\prime T} \mathbf{T}\right]=\mathbf{T}^{T} E\left[\mathbf{X}^{\prime} \mathbf{X}^{\prime T}\right] \mathbf{T}=\mathbf{T}^{T} \mathbf{C}^{\prime} \mathbf{T} \equiv[\lambda]
$$

Thus, as $[\lambda]$ is diagonal, the transformed variables $\mathbf{Y}$ are uncorrelated as requested. They have zero mean but non unitary variances.

Finally, if $\left[\sigma_{X}\right]$ is the diagonal matrix of the standard deviations of $\mathbf{X}$ and $\left[\mu_{X}\right]$ the vector of the means, the original variables can be expressed as

$$
\mathbf{X}=\left[\sigma_{X}\right] \mathbf{T Y}+\left[\mu_{X}\right]
$$

because $\mathbf{T}$ is orthogonal and thus $\mathbf{T}^{-1}=\mathbf{T}^{T}$.

Similarly to the Rosenblatt transformation, the transformation (4.24) can be used to reformulate the problem in the space of transformed coordinates $\mathbf{Y}$. Once the MPP is found, its coordinates in the original space could be obtained by (4.27). Most commonly the transformation is embedded in the FORM search algorithm. In this case the derivatives of the performance function in the transformed space are required:

$$
\frac{\partial g}{\partial Y_{i}}=\sum_{j=1}^{n} \frac{\partial g}{\partial X_{j}^{\prime}} \cdot \frac{\partial X_{j}^{\prime}}{\partial Y_{i}}=\sum_{j=1}^{n} \sigma_{X_{j}} \frac{\partial g}{\partial X_{j}} \cdot T_{j i}
$$

In Fig. 4.14 the effect of the orthogonal transformation on the limit state for the test case is plotted; only gust factor and gust length are considered and are assumed to be correlated with a correlation coefficient equal to 0.5 . 


\section{Reliability evaluation}

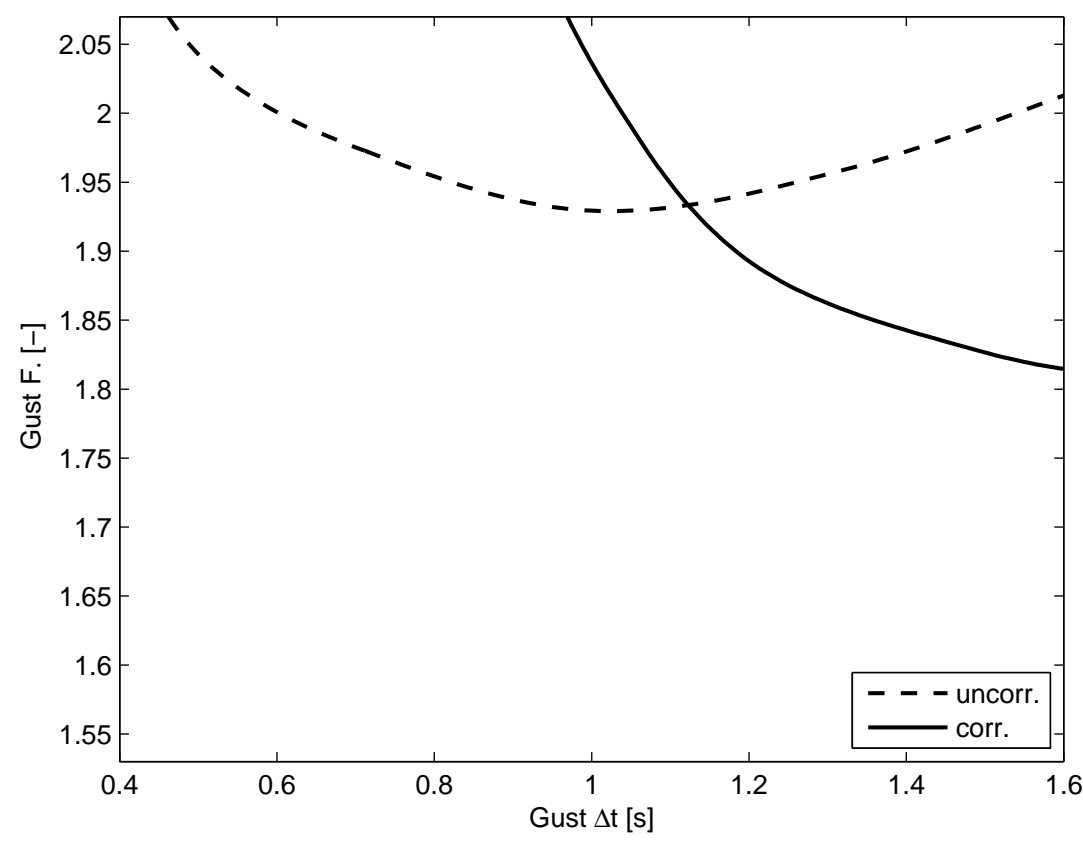

Figure 4.14: Example of limit state for uncorrelated variates (dashed line) and correlated variates after the orthogonal transformation (continuous line).

\section{Comparison}

In Fig. 4.15 the probability of failure over the mean wind velocity is plotted for exemplary scenarios of correlated and nonnormal (triangular) distributed variables. They were computed respectively with the orthogonal and Rosenblatt transformation. It can be well seen that especially the nonnormality of the distributions have significant influence on the probability of failure. It follows that this topic should be given large attention when models for the uncertainty and variability are defined.

\subsubsection{Sampling based techniques}

\section{Monte Carlo}

The evaluation of the probability of failure by the integral (4.1) can also be performed by Monte Carlo (MC). To this aim the equation must be rewritten as:

$$
p_{F}=\int_{\Omega} I[g(\mathbf{x}) \leq 0] f_{\mathbf{X}}(\mathbf{x}) \mathrm{d} \mathbf{x} \equiv \mathrm{E}_{f}\{I[\ldots]\},
$$

where

$$
I[y]=\left\{\begin{array}{lll}
1 & : & y=\text { true } \\
0 & : & y=\text { false }
\end{array} .\right.
$$

The domain of integration is now the whole space $\Omega$, not only the failure region $\Omega_{F}$, because the failure region is taken into account by the function $I$. The probability 


\subsection{Evaluation of the probability of failure}

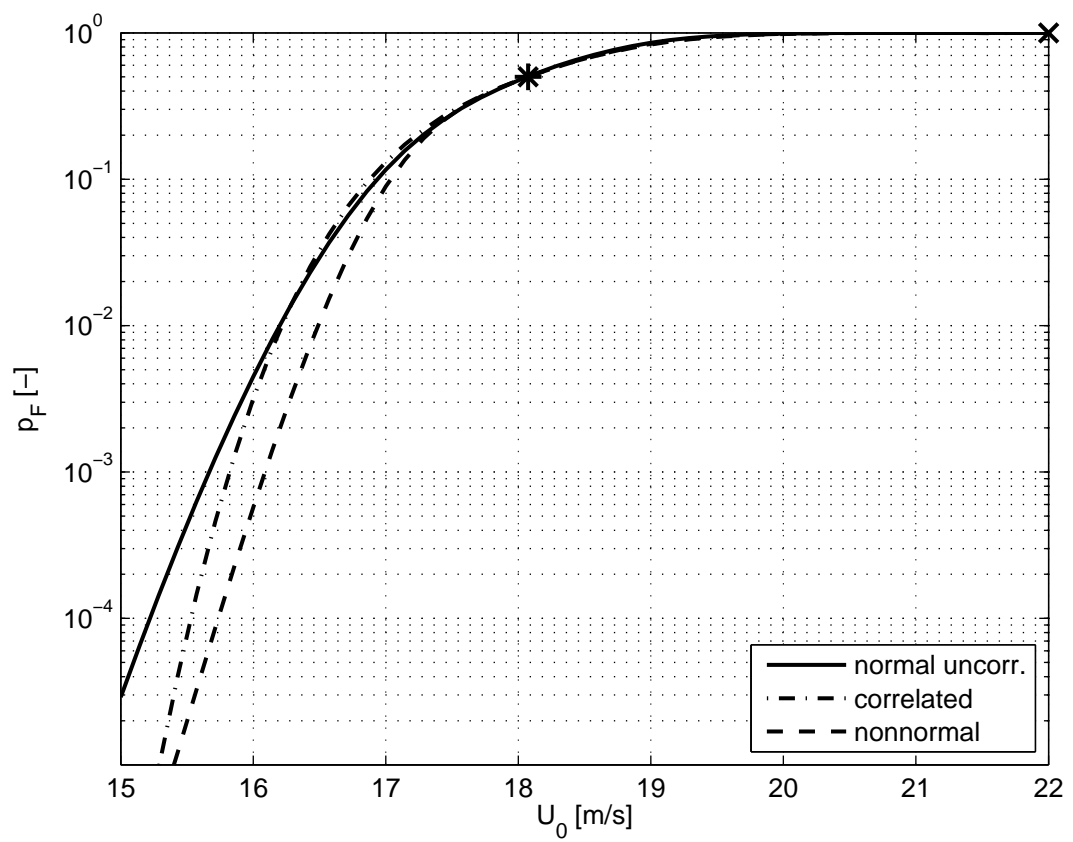

Figure 4.15: Comparison of the probability of failure as a function of the mean wind velocity for normal uncorrelated, normal correlated and nonnormal uncorrelated variables.

of failure can be interpreted as expected value of $I$; if $\mathbf{x}$ is sampled according to the distributions $f_{\mathbf{X}}$ and $N$ samples $\mathbf{x}_{j}$ are considered, the so called crude Monte Carlo estimate of (4.29) is:

$$
\hat{p}_{F}=\frac{1}{N} \sum_{j=1}^{N} I\left[g\left(\mathbf{x}_{j}\right) \leq 0\right] .
$$

Monte Carlo is a very powerful method because the system, i.e. the performance function, is handled as a black-box and no assumptions have to be made at all. On the other hand, the application of MC techniques to reliability related tasks is problematic because the integral to be computed, i.e. the probability of failure, assumes very low values in the range of major interest, calling for very large sample size. The estimation of the accuracy and the remedy offered by the variance reduction techniques, in particular the importance sampling, are addressed below.

For the application of Monte Carlo to the case of correlated variables the joint PDFs must be available in order to generate the samples but such information is rarely available because the covariance matrix is at most given. Consequently the application of Monte Carlo is confined in practice to the case of uncorrelated variables. On the contrary the application of Monte Carlo to nonnormal uncorrelated variables does not require any special attention because the generation of samples with arbitrary distributions can be nowadays done by computer codes. 


\section{Reliability evaluation}

\section{Accuracy and variance reduction}

The estimation of the accuracy of a Monte Carlo simulation is still an open problem and approximations are required, see for example [Rub81, Mel99]. The Shooman's formula for the error $e$ between computed and real probability of failure is often used:

$$
e=2 \sqrt{\frac{1-p_{F}}{N \cdot p_{F}}}
$$

A sample size $N=10^{5}$ is thus an appropriate value for reliability problems $(20 \%$ accuracy with $95 \%$ confidence at $p_{F}=10^{-3}$ ). This estimation shows that the use of response surfaces to reduce the computational effort is almost mandatory when the performance function is only known implicitly, i.e. by a simulation model. Nonetheless, the computational effort is still not bearable, because in the crosswind stability analysis the probability of failure has to be evaluated not only once but many times, corresponding to different values of the parameters $U_{0}$ and $V$.

A reduction of the effort, i.e. of the sample size $N$ needed to obtain a given accuracy level, can be obtained by variance reduction techniques. A large number of such techniques are based on the use of quasi-random sequences, like descriptive sampling. However, the improvement in efficiency in term of convergence performances, sampling size, etc. that can be achieved is not large and depends strongly on the specific application. For the special case of reliability analysis importance sampling, [Rub81], is the most adequate technique and is widely used in practice.

The importance sampling concept derives from a simple modification of (4.29):

$$
p_{F}=\int_{\Omega} I[g(\mathbf{x}) \leq 0] \frac{f_{\mathbf{X}}(\mathbf{x})}{h_{\mathbf{X}}(\mathbf{x})} h_{\mathbf{X}}(\mathbf{x}) \mathrm{d} \mathbf{x} \equiv \mathrm{E}_{h}\left\{I[\ldots] \frac{f_{\mathbf{X}}}{h_{\mathbf{X}}}\right\},
$$

$h_{\mathbf{X}}$ being called importance sampling distribution. In (4.33) it is stresed that the expected value $E$ refers now to $h_{\mathbf{X}}$. The Monte Carlo estimate of (4.33) is:

$$
\hat{p}_{F}=\frac{1}{N} \sum_{j=1}^{N}\left\{I\left[g\left(\mathbf{x}_{j}\right) \leq 0\right] \frac{f_{\mathbf{X}}\left(\mathbf{x}_{j}\right)}{h_{\mathbf{X}}\left(\mathbf{x}_{j}\right)}\right\}
$$

where the samples $\mathbf{x}_{j}$ are distributed according to $h_{\mathbf{X}}$ and not to $f_{X}$. In this way the distribution of the samples is decoupled from the distribution of the variables: a conventional sampling strategy is used, but the sample distribution $h_{\mathbf{X}}$ can be chosen so that the samples are concentrated in the region that actually determines the integral.

The sample distribution which optimises the efficiency can be proved to be:

$$
h_{\mathbf{X}}^{o p t}=\frac{I[g(\mathbf{x}) \leq 0] f_{\mathbf{X}}(\mathbf{x})}{p_{F}},
$$

which is the distribution of the variables $\mathbf{X}$ truncated in the failure region $\Omega_{F}$ and scaled with $p_{F}$. This choice is clearly not possible because the failure region is known only implicitly and $p_{F}$ is the quantity to be computed. A possible choice, which was also 


\subsection{Evaluation of the probability of failure}

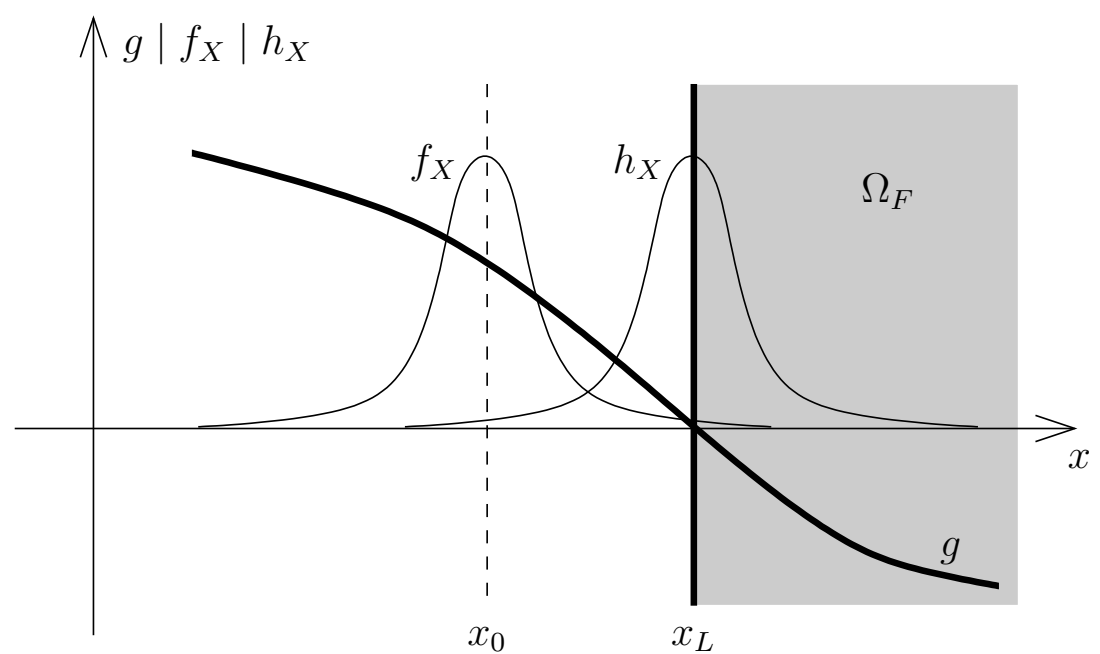

Figure 4.16: Importance sampling. $h_{X}$, which is $f_{X}$ translated to $x_{L}$, is a possible importance sampling distribution.

used in this work, is the distribution $f_{X}$ translated to the Most Probable Point which must have been computed when the Monte Carlo simulation is started. This is shown, for the one dimensional example, in Fig. 4.16. More efficient techniques for the choice of the sampling distribution have been proposed, for example the adaptive sampling technique, [Buc88], but they have been not considered in this work.

In Fig. 4.17 a typical convergence history for a MC run with and without importance sampling is reported. The estimate of the probability of failure without importance sampling (dashed line) is completely useless because only every 500-th (or more) sample falls in the failure region, causing huge steps in the estimate.

Finally, it must be mentioned that the estimation of the accuracy when using importance sampling is more difficult than in the crude Monte Carlo. Plots of the probability of failure over the iteration like Fig. 4.17 are often the most appropriate even though empirical way to check the convergence.

\subsubsection{Other tools}

\section{Metamodels}

In the reliability analysis the performance function is defined implicitly by the multibody simulation code, i.e. every evaluation of the performance function requires a simulation. Even though the computational effort for one MBS simulation is not very large, many simulations have to be performed in the overall process, especially for Monte Carlo; a comparison of the effort required for the different methods is reported in Sec. 4.2.5. A possible solution to this problem is offered by metamodels, [SLC01, $\mathrm{S}^{+} 01$ ], which substitute the original model with a more simple and necessarily less accurate one. The advantage with respect to a model reduction is that metamodels handle the original model as a black-box, i.e. the original model has not to be manipulated and its internal 


\section{Reliability evaluation}

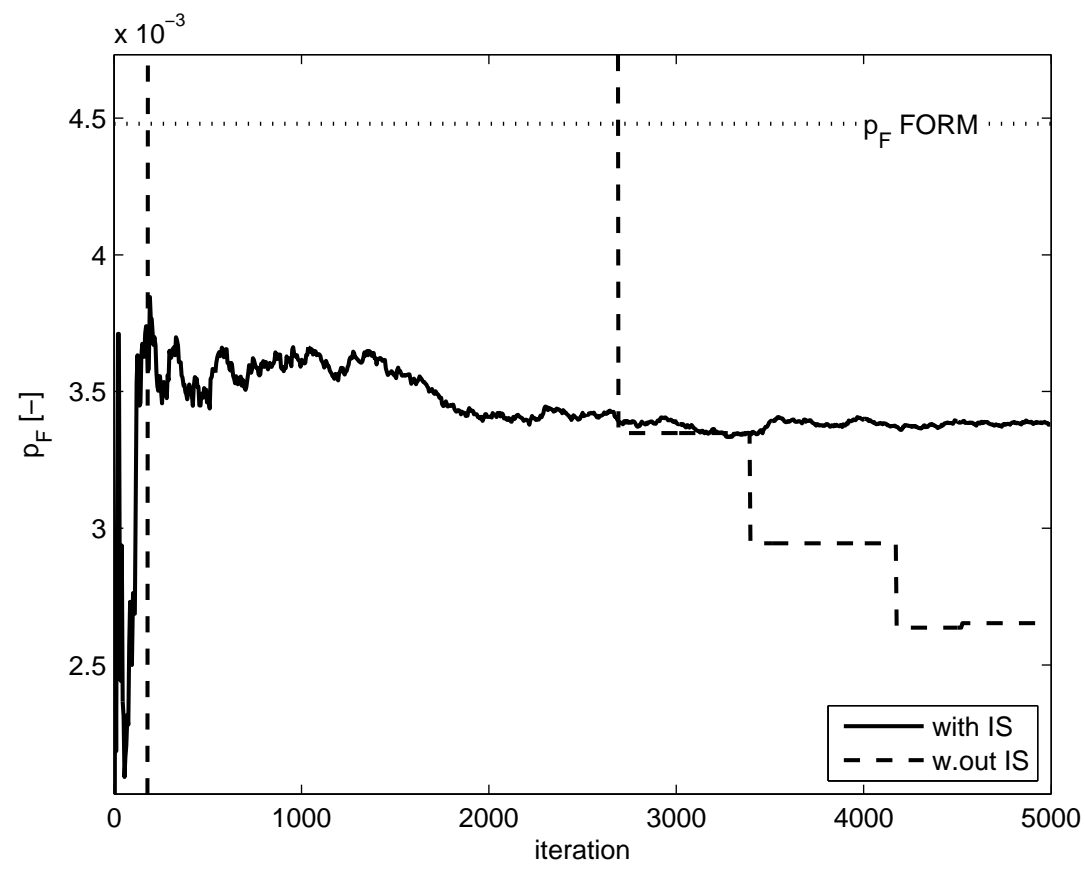

Figure 4.17: Probability of failure over iteration for a Monte Carlo run with and without importance sampling. The probability of failure computed with FORM is also plotted for comparison.

functionality has not to be known at all.

A widely used kind of metamodels are response surfaces, [MGH02]. In this approach the performance function is evaluated in a preprocessing phase at given fixed points, the number of which is comparatively low. Then, in the reliability analysis, the MBS simulation is substituted by an interpolation between the already computed values of the performance function. As an interpolation, although multidimensional, is computationally not expensive, the overall effort is strongly reduced. On the other hand, the number of computations needed to obtain the surface is low only if the behaviour of the original model can be well covered by low order interpolation scheme. In this work spline interpolation on a regularly spaced grid was used.

For the reliability analysis the use of response surfaces is problematic. Surfaces centred on the nominal values of the variables are inefficient, because only the region of interest around the limit state, which is not known a priori, has to be modelled accurately. On the other hand, high order surfaces over the whole space are expensive. A possible solution to the problem can be found in iterative response surfaces, [BB90]. The results of the reliability analysis on an initial quadratic surface centred on the mean values are used to estimate the limit state, around which a new local quadratic response surface is computed; the process is iterated until convergence. 


\subsection{Evaluation of the probability of failure}

\section{Screening}

The computational effort for the reliability analysis depends strongly on the number of variables, i.e. on the dimension of vector $\mathbf{X}$. An effective way to reduce this dimension is to perform some simulations in the preprocessing phase which identify the most influential variables. Such simulations are usually denoted as screening and are performed according to conventional schemes from Design of Experiments, [MGH02].

In Fig. 4.18 results of screening experiments are reported. The conventional fractional factorial design was used and slightly modified to give an overview on nonlinearities and interactions between the parameters without increasing excessively the number of runs. For every variable a reasonable range of variation was set and the wheel unloading $Q^{\prime}$ was computed at the nominal value and at the extremes of the range. In each plot the thick line refers to case in which all the other variables are fixed at their nominal values, whereas the thin lines to the case in which the other variables assume every possible combination of lower and upper bounds of the respective range.
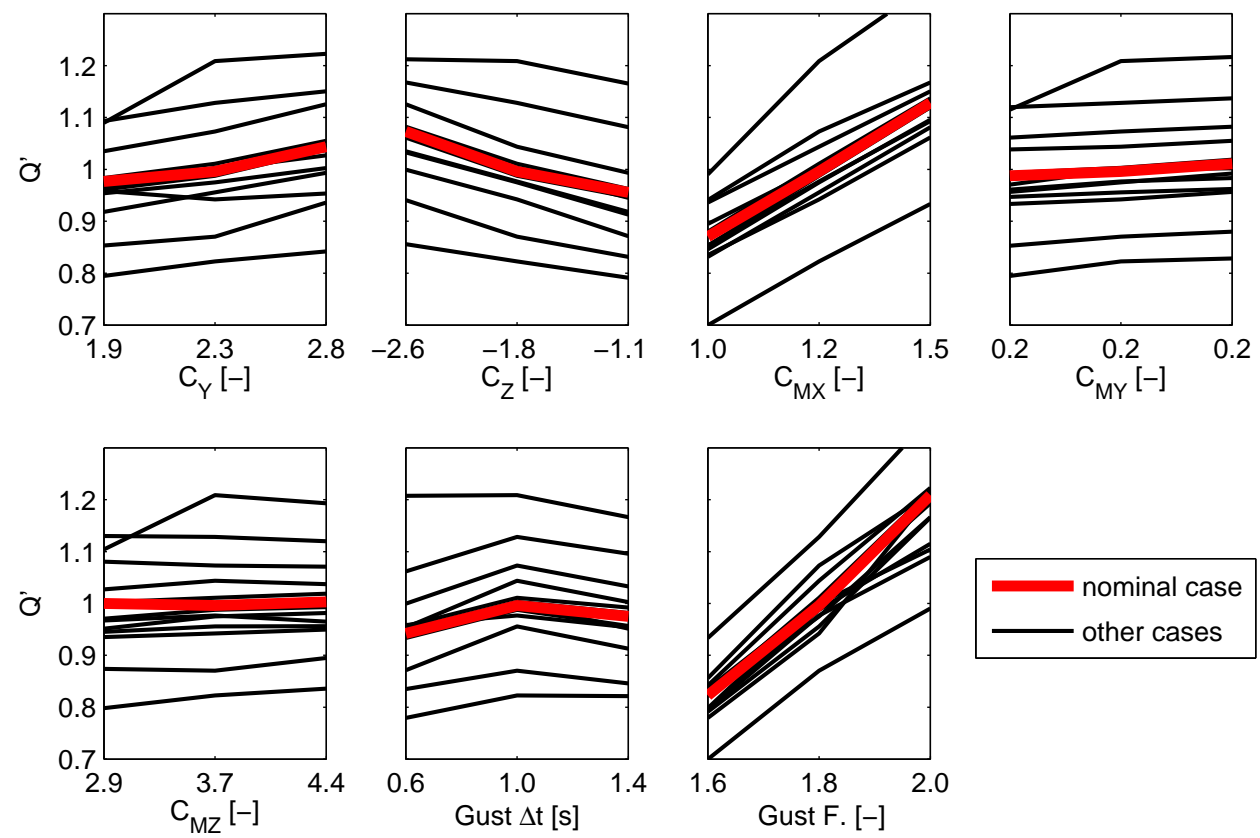

Figure 4.18: Results of screening experiments. The thick lines refer to case in which all the other variables are fixed at their nominal values, whereas the thin lines to the case in which the other variables assume every possible combination of lower and upper bounds.

It can be clearly seen that the aerodynamic roll moment coefficient $C_{M X}$ and the gust factor GustF are the most influential parameters, followed by aerodynamic lift and side force coefficients, $C_{Z}$ and $C_{Y}$. Strong nonlinearities are not present and only the gust length Gust $\Delta t$ is not monotone; this is also the reason why the gust length, which is not very influential, was included in this work in the vector $\mathbf{X}$. Finally, the interactions between the parameters seems to be not very strong, as the thin lines show, for every case, a similar trend to the thick ones. 


\section{Reliability evaluation}

\subsubsection{Comparison of the methods}

A comparison between FORM, Mean Value and Monte Carlo for a single wind velocity $\left(U_{0}=16 \mathrm{~m} / \mathrm{s}\right)$, including the CPU time, is reported in Table 4.1. The result obtained with Monte Carlo can be assumed to be very close to reality and can be thus used as a reference. It follows from Table 4.1 that the accuracy of FORM is not very good. The fact that the MV result is closer to MC than FORM is a coincidence because the MV method is based on a poorer approximation then FORM.

As to the CPU time, the excessive effort required by Monte Carlo is evident. It must be noted that the computational time for the creation of the response surface depends strongly on the used surface. The spline interpolation used in this work leads to $T_{R S} \simeq 6000 \mathrm{~s}$ but less accurate models, like a global cubic function, would have also produced similar results.

To sum up, the integrated approach for Monte Carlo proposed above (importance sampling on response surface centred at the MPP), seems to be the best compromise when the computational effort can be borne. The obvious condition is that the behaviour of the system can be well reproduced with response surfaces.

Table 4.1: Comparison of the methods and computational effort with and without Response Surfaces for one evaluation of the probability of failure (test case with $U_{0}=16 \mathrm{~m} / \mathrm{s}$ ). $T_{R S}$ denotes the effort for the creation of the response surface.

\begin{tabular}{c||c|c|c} 
& MC (IS) & FORM & MV \\
\hline \hline$p_{F}$ & 0.002600 & 0.004707 & 0.002434 \\
$\begin{array}{c}\text { CPU time } \\
\text { with RS }\end{array}$ & $60 \mathrm{~s}\left(+T_{R S}\right)$ & $2 \mathrm{~s}\left(+T_{R S}\right)$ & $<1 \mathrm{~s}\left(+T_{R S}\right)$ \\
$\begin{array}{c}\text { CPU time } \\
\text { w.out RS }\end{array}$ & $\simeq 50000 \mathrm{~s}$ & $\simeq 1500 \mathrm{~s}$ & $\simeq 150 \mathrm{~s}$
\end{tabular}

\subsection{Probabilistic sensitivity analysis}

In this section the sensitivity of the reliability and the general system performances with respect to the uncertainty in the parameters is studied. This kind of sensitivity is often denoted as probabilistic sensitivity analysis, to be distinguished from the conventional deterministic sensitivity in which variations of the parameter values, and not of their statistics, are studied. Moreover, it is a global sensitivity, because it is influenced by the 


\subsection{Probabilistic sensitivity analysis}

system behaviour on the whole parameter space and not only in proximity of a point as in the deterministic case.

It must be pointed out that the final aim of the techniques presented here is not to compute exact absolute values of the sensitivity but to obtain some information on the relative importance of the variables and their statistics. This kind of result determines on the one hand where high accuracy in the formulation of the models and in the determination of the parameters must be striven for. On the other hand, as far as the parameters which can be influenced by design are concerned, it gives indications for the improvement of future vehicles.

\subsubsection{Reliability sensitivity}

The reliability sensitivity is the reliability of the probability of failure with respect to the uncertainty in the parameters. It can be carried out on the basis of the FORM approximation or the sampling approach. With regard to the former, only the main results are reported; for a derivation see [DM96].

\section{FORM approach}

Importance factors The direction cosines $\alpha_{i}$ of the vector from the origin to the MPP are denoted as importance factors and have been already introduced in Sec. 4.2.1. As the reliability index $\beta$ is equal to the norm of such vector, the importance factors are proportional to the contribution of each variable to the reliability index and thus to the probability of failure.

Another interpretation of the importance factors can be found observing that the stochastic variable defined by the performance function, $G=g(X)$, can be written according to the FORM linearisation as:

$$
G=\beta-\alpha \cdot \mathbf{X}^{\prime}
$$

This is well comprehensible considering the one dimensional example of Fig. 4.5, $\beta$ being equal to $\left(x_{L}-x_{0}\right)$. The variance of $G$ is then:

$$
\operatorname{Var}[G]=\alpha_{1}^{2}+\ldots+\alpha_{n}^{2}=1
$$

because of the definition of the reduced variables $\mathbf{X}^{\prime}$, cp. (4.12). It follows that the square of the importance factor $\alpha_{i}^{2}$ is the contribution of $\mathbf{X}_{i}$ to the variance of the limit state.

The importance factors for the test case are plotted in Fig. 4.19, bottom; they can also be immediately derived from the position of the most probable point in the MPP locus, see Fig. 4.7 on page 63. The fact that the importance factors are almost constant suggests a linear system behaviour.

Sensitivity of the reliability index The sensitivity of the reliability index $\beta$ with respect to the parameters is now examined. Consider a generic parameter $p$ which influences $\beta$ 


\section{Reliability evaluation}
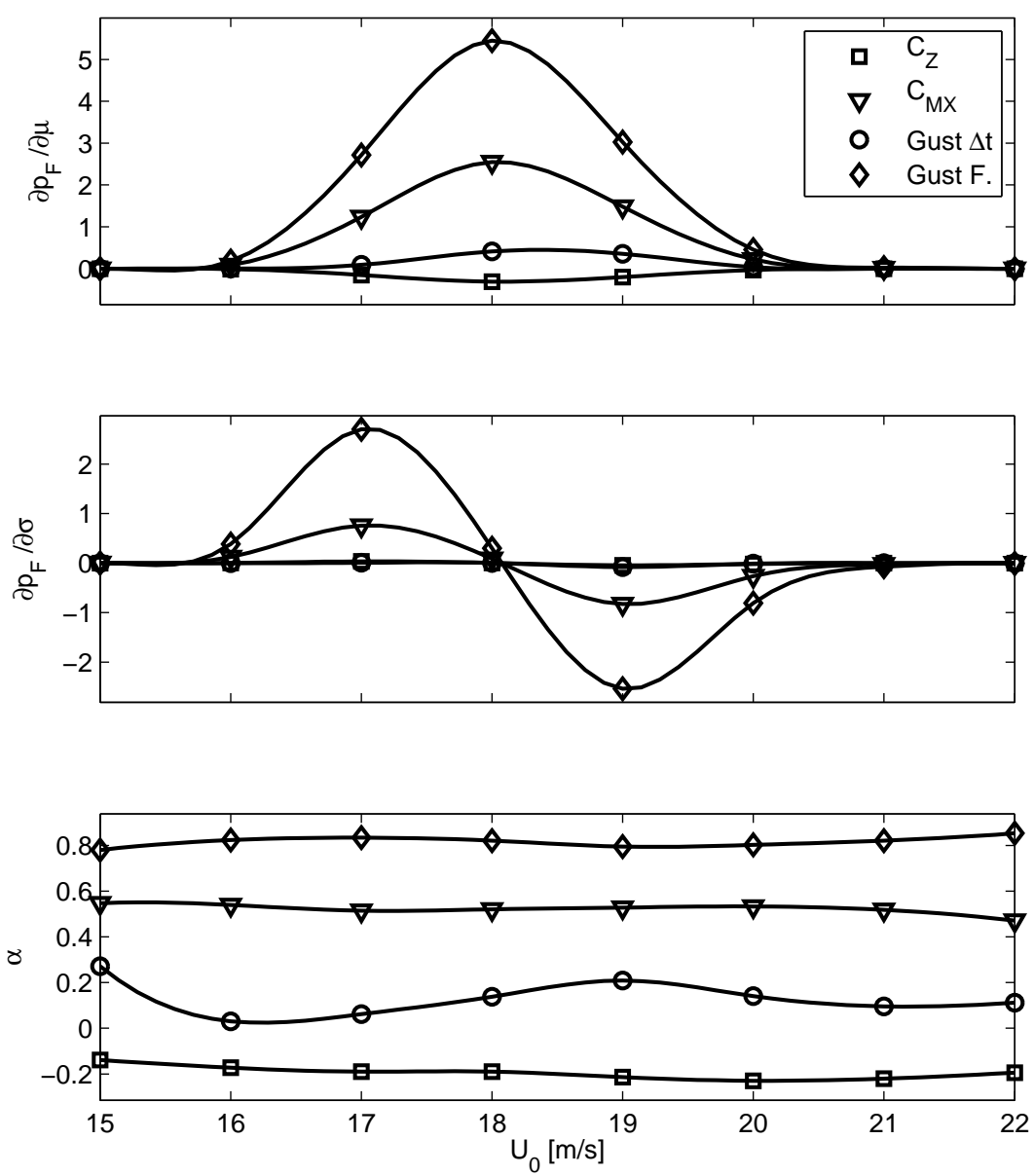

Figure 4.19: Derivatives of the probability of failure with respect to mean and standard deviation (top, middle) and importance factors (bottom) for the test case with normal uncorrelated parameters.

but not necessarily the limit state $g(\mathbf{x}) ; p$ can be a deterministic parameter such as $U_{0}$ and $V$, the mean value or the variance of a variable $\mathbf{X}_{i}$ etc. In the FORM approach the derivative of $\beta$ with respect to $p$ can be shown to be:

$$
\frac{\partial \beta}{\partial p}=\left.\frac{1}{\|\nabla g\|} \frac{\partial g}{\partial p}\right|_{\mathbf{x}^{\prime}=\mathbf{x}^{\prime *}}
$$

to which follows that the derivative of the probability of failure $p_{F}=\Phi(-\beta)$ is:

$$
\frac{\partial p_{F}}{\partial p}=-\varphi(\beta) \frac{\partial \beta}{\partial p}
$$

Two cases must be now considered separately:

- $p$ deterministic parameter. Such a parameter, e.g. the driving velocity $V$ or the wind velocity $U_{0}$, affects the limit state, cp. (4.5). The derivative $\partial g / \partial p$ needed in 


\subsection{Probabilistic sensitivity analysis}

(4.38) is simply:

$$
\frac{\partial g^{\prime}\left(\mathbf{x}^{\prime}, p\right)}{\partial p}=\frac{\partial g(\mathbf{x}, p)}{\partial p},
$$

and can be computed by finite differences when $g$ is defined implicitly. However, in the crosswind stability task the whole relation $p_{F}\left(U_{0}, V\right)$ has to be established and thus the computation of $\partial p_{F} / \partial U_{0}$ or $\partial p_{F} / \partial V$ with (4.39) does not offer any additional benfit.

- $p$ distribution parameter. This kind of parameters, such as mean and variance of the parameters, do not affect the limit state but the reliability index. It can be shown that in this case (4.38) reads:

$$
\frac{\partial \beta}{\partial p}=\left.\boldsymbol{\alpha} \cdot \frac{\partial \mathbf{x}^{\prime}}{\partial p}\right|_{\mathbf{x}^{\prime}=\mathbf{x}^{\prime *}},
$$

where $\boldsymbol{\alpha}$ is the vector of the director cosines. The derivative on the right hand side can also be written as:

$$
\frac{\partial \mathbf{x}^{\prime}}{\partial p}=\frac{\partial T(\mathbf{x} ; p)}{\partial p}
$$

$T$ being the transformation from the original to the reduced space, cp. (4.11). For normal uncorrelated variates $T(\mathbf{x} ; p)$ reduces to $(4.12)$; considering $p=\mu_{i}$ and $p=\sigma_{i},(4.41)$ reads:

$$
\begin{aligned}
\frac{\partial \beta}{\partial \mu_{i}} & =-\frac{\alpha_{i}}{\sigma_{i}} \\
\frac{\partial \beta}{\partial \sigma_{i}} & =-\frac{\alpha_{i}}{\sigma_{i}} x_{i}^{*} .
\end{aligned}
$$

The first of the relations offers another interpretation of the direction cosines $\alpha_{i}$, for the case of normal uncorrelated variables, as derivatives of the reliability index with respect to the mean values, adimensionalised by the respective standard deviations.

For correlated normal variates, when $T$ is the transformation matrix described in 4.2.2, the computation of (4.42) can still be performed analytically. In this case (4.41) is:

$$
\begin{aligned}
\frac{\partial \beta}{\partial \mu_{i}} & =\sum_{j=1}^{n} \alpha_{j} \frac{T_{i j}}{\sigma_{i} \lambda_{j}} \\
\frac{\partial \beta}{\partial \sigma_{i}} & =\sum_{j=1}^{n} \alpha_{j} \frac{T_{i j}}{\sigma_{i} \lambda_{j}} x_{i}^{*} .
\end{aligned}
$$

On the contrary, in the most general case, e.g. when the Rosenblatt transformation (4.20) is used, the computation of (4.42) has to be performed numerically by finite differences. 


\section{Reliability evaluation}

In Fig. 4.19, top and middle, the derivatives for the test case with normal uncorrelated parameters according to (4.43) are plotted over the mean wind velocity, bringing out that the gust factor and roll moment coefficient are the most influential parameters. The derivatives show the typical developing which can be well interpreted considering the one dimensional example of Fig. 4.3 and remembering the probability of failure is the area spanned under the PDF lying in the failure region $\left(x>x_{L}\right)$. For example, when the mean is close to the limit state $\left(x_{0} \simeq x_{L}, p_{F} \simeq 0.5\right.$, corresponding to $U_{0} \simeq 18 \mathrm{~m} / \mathrm{s}$ in Fig. 4.19) the derivative $\partial p_{F} / \partial \mu$ has a maximum, because any variation of the mean, i.e. any "translation" of the PDF, significantly varies the area lying in the failure region. At the same point $\partial p_{F} / \partial \sigma$ is equal to zero because any variation of the variance, i.e. any "stretching" or "compression" of the PDF, does not change the fact that half the area under the PDF is in the failure region and half outside. On the contrary, when the mean is far from the limit state, e.g. at large values of $U_{0}$, the derivatives have low values, because any variation of mean and variance changes not much less the area spanned by the PDF lying in the failure region.

\section{Sampling based approach}

To assess the effect of a very generic variation of the Probability Density Function, say $\Delta f_{\mathbf{X}}$, on the probability of failure a sampling based approach can be used. To this aim two Monte Carlo runs have to be performed: one with the original distribution function $f_{\mathbf{X}}$ and one with the modified one $(f+\Delta f)_{\mathbf{X}}$. A key point is that, for the comparison to make sense, the same sample set have to be used for both runs, which can be interpreted as a kind of correlated sampling, see [Rub81]. The probability of failure computed with the second run is, cp. (4.34):

$$
\widehat{p_{F}+\Delta p_{F}}=\frac{1}{N} \sum_{j=1}^{N}\left\{I\left[g\left(\mathbf{x}_{j}\right) \leq 0\right] \frac{(f+\Delta f)_{\mathbf{X}}\left(\mathbf{x}_{j}\right)}{h_{\mathbf{V}}\left(\mathbf{x}_{j}\right)}\right\},
$$

where $p_{F}+\Delta p_{F}$ is the probability of failure resulting from the modified PDF $f+\Delta f$. Using the same sample a significant reduction of the computational effort is achieved: in the second run with the modified PDF the evaluation of the performance function at the sample points $g\left(\mathbf{x}_{j}\right)$, which is the most expensive part of the process, is already available from the first run. It follows that only the counting process, i.e. the evaluation of $I[\ldots]$, and the evaluation of the modified PDF $(f+\Delta f)_{\mathbf{X}}$ have to be computed, because the values of the importance sampling distribution $h_{\mathbf{V}}\left(\mathbf{x}_{j}\right)$ are already available too. The sensitivity can finally be evaluated as:

$$
\frac{\partial p_{F}}{\partial f_{\mathbf{X}}} \simeq \frac{\Delta p_{F}}{\Delta f_{\mathbf{X}}}
$$

having assumed that the generic variation $\Delta f_{\mathbf{X}}$ (and the corresponding infinitesimal $\left.\partial f_{\mathbf{X}}\right)$ can be properly defined and quantified.

In Fig. 4.20 the sensitivities computed with FORM and Monte Carlo for $U_{0}=16 \mathrm{~m} / \mathrm{s}$ are plotted. Assuming that the Monte Carlo results are very close to the real values it 


\subsection{Probabilistic sensitivity analysis}

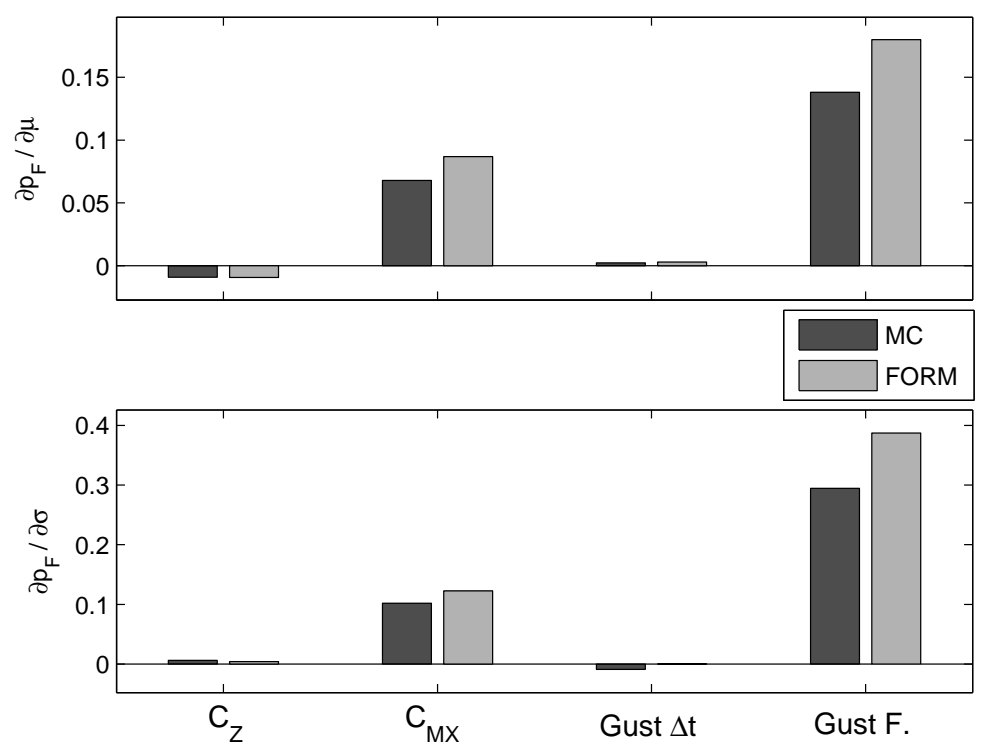

Figure 4.20: Sensitivity of the probability of failure with respect to mean $(t o p)$ and variance (bottom) of the parameters computed with MC and FORM for $U_{0}=16 \mathrm{~m} / \mathrm{s}$.

can be seen that the FORM approach gives an acceptable approximation. However, as in the case of the simple evaluation of $p_{F}$, the use of Monte Carlo for the whole range of mean wind and driving velocities leads to unbearable computational effort.

\subsubsection{Response sensitivity}

In this section the sensitivity of the system response with respect to the uncertainties in the parameters is studied independently from the reliability topic. For the crosswind stability task this means that, given the uncertainties in the parameters, the corresponding uncertainty in the value of wheel unloading is looked for. This kind of information is of fundamental importance because one goal of the vehicle design process should be to have a system response without uncertainties; in this case safety limits would be no more necessary. In practice, this is not possible and the goal is, more realistically, to design a system having a behaviour as insensitive as possible to the uncertainties in the parameters.

\section{Uncertainty propagation}

The statistics of the variable $G$ defined by the performance function $g(\mathbf{x})$ can be computed analytically only for few cases. In the general case of nonlinear $g(\mathbf{x})$ and/or nonnormal correlated variates $\mathbf{X}$, only approximated results are possible. Many methods can be used in this case, either on the original model or on a metamodel. The most basic but also computationally inexpensive method is the Taylor expansion; the resulting expressions are identical to those presented in the context of the Mean Value method, 


\section{Reliability evaluation}

Eqs. (4.8) and (4.9). Monte Carlo can also be used to assess the sensitivity when the computed samples are evaluated by regression analysis or similar techniques. In this work such standard methods for uncertainty propagation have not been considered.

\section{Variance based sensitivity}

The variance based sensitivity, [HS96, Sob01], aims to quantify by means of some sensitivity indices the contribution of the variables and their interactions to the response variance. Due to the fact that the variance based sensitivity is a relatively recent technique, not widely spread outside the statistician community, some details are reported in what follows.

Starting point is the decomposition of the variance of $G=g(\mathbf{X})$. Under the assumption that the variables $X_{i}$ are statistically independent, the variance can be written as:

$$
\operatorname{Var}[G]=V=\sum_{i=1}^{n} V_{i}+\sum_{i=1}^{n} \sum_{j=i+1}^{n} V_{i j}+\ldots+V_{i \ldots n}
$$

where the first order terms $V_{i}$ are the contributions of the variables $X_{i}$ alone to the total variance; the second order terms $V_{i j}$ are the additional contributions due to the interaction between $X_{i}$ and $X_{j}$, i.e. an extra amount of variance due to $X_{i}$ and $X_{j}$ additional to the contributions $V_{i}$ and $V_{j}$, and so on. More formally:

$$
\begin{aligned}
V_{i} & =\operatorname{Var}_{i}\left[\mathrm{E}_{\sim i}\left(G \mid X_{i}\right)\right] \\
V_{i j} & =\operatorname{Var}_{i j}\left[\mathrm{E}_{\sim\{i j\}}\left(G \mid X_{i}, X_{j}\right)\right]-V_{i}-V_{j} .
\end{aligned}
$$

In the equations it was emphasised that the variances are computed with respect to $X_{i}$, $X_{i j}$ etc. whereas the expected value operators refer to all variables except $X_{i}, X_{i j}$, etc. Considering for simplicity's sake only the first of (4.48), an equivalent interpretation can be given observing that using an established statistical identity $V_{i}$ can be written as:

$$
V_{i}=\operatorname{Var}_{1 \ldots n}[G]-\mathrm{E}_{i}\left\{\operatorname{Var}_{\sim i}\left[G \mid X_{i}\right]\right\}
$$

with similar notation for the $\mathrm{E}[\ldots]$ and $\operatorname{Var}[\ldots]$ operators. This shows that $V_{i}$ is the reduction of the variance of $G$ which arises when the variable $X_{i}$ is no more stochastic but assumes a fixed value; on the other hand, such fixed value, $x_{i}$, is arbitrary and thus the expected value of $\operatorname{Var}_{\sim i}\left[G \mid X_{i}\right]$ has to be taken.

The sensitivity indices can then be defined as:

$$
\begin{aligned}
S_{i}=\frac{V_{i}}{V} & 1 \leq i \leq n \\
S_{i j}=\frac{V_{i j}}{V} & 1 \leq i \leq j \leq n
\end{aligned}
$$




\subsection{Probabilistic sensitivity analysis}

$S_{i}$ being called main sensitivity index of $x_{i}$ while $S_{i j \ldots}$ is the interaction sensitivity index of $x_{i}, x_{j}, \ldots$. As

$$
\sum_{i=1}^{n} S_{i}+\sum_{i=1}^{n} \sum_{j=i+1}^{n} S_{i j}+\ldots=1
$$

the indeces can be interpreted as fractions of the total variance due respectively to the single variables, to the interaction between couples of variables and so on.

Different techniques have been proposed for the computation of the indices. In this work the sampling based one proposed by Sobol in [Sob01] is used. First of all, the performance function is decomposed into terms of different dimensions:

$$
g(\mathbf{x})=g_{0}+\sum_{i=1}^{n} g_{i}\left(x_{i}\right)+\sum_{i=1}^{n} \sum_{j=i+1}^{n} g_{i j}\left(x_{i}, x_{j}\right)+\ldots+g_{i \ldots n}(\mathbf{x}) \quad,
$$

The first order terms $g_{i}$ are called main effects, whereas the higher order terms are called interaction effects. For simplicity's sake suppose that the domain of $\mathbf{x}$ is

$$
K^{n}=\left\{\mathbf{x} \mid 0 \leq x_{i} \leq 1 ; i=1 \ldots n\right\} \quad .
$$

Moreover, the stochastic variables $\mathbf{x}$ are assumed to be uniformly distributed in $K^{n}$. This assumption is not restrictive because the interest lies in the general behaviour of the system, thus independently from the (statistic) properties of its parameters.

The decomposition (4.52) requires that $g_{0}$ is constant and the integral of the generic summand $g_{i \ldots m}\left(x_{i}, \ldots, x_{m}\right)$ over any of its variables is zero:

$$
\int_{0}^{1} g_{i \ldots m}\left(x_{i}, \ldots, x_{m}\right) \mathrm{d} x_{k}=0 \quad, \quad i \leq k \leq m \leq n
$$

It follows that the orthogonality property for two different generic terms $g_{i \ldots m}$ and $g_{i^{\prime} \ldots m^{\prime}}$ holds:

$$
\int_{0}^{1} \ldots \int_{0}^{1} g_{i \ldots m}\left(x_{i}, \ldots, x_{m}\right) g_{i^{\prime} \ldots m^{\prime}}\left(x_{i^{\prime}}, \ldots, x_{m^{\prime}}\right) \mathrm{d} \mathbf{x}_{i i^{\prime} \ldots m m^{\prime}}=0 .
$$

Stopping the expansion to the second order, the addends of the decomposition (4.52) are then:

$$
\begin{aligned}
& g_{0}=\mathrm{E}[G] \\
& =\int_{K^{n}} g(\mathbf{x}) \mathrm{d} \mathbf{x} \\
& g_{i}\left(x_{i}\right)=\mathrm{E}\left[G \mid X_{i}\right]-\mathrm{E}[G] \quad=\int_{K^{n-1}} g(\mathbf{x}) \mathrm{d} \mathbf{x}_{\sim i}-g_{0} \\
& g_{i j}\left(x_{i}, x_{j}\right)=\mathrm{E}\left[G \mid X_{i}, X_{j}\right]-g_{i}-g_{j}-\mathrm{E}[G]=\int_{K^{n-2}} g(\mathbf{x}) \mathrm{d} \mathbf{x}_{\sim\{i j\}}-g_{i}-g_{j}-g_{0} \quad,
\end{aligned}
$$

$\mathbf{x}_{\sim i}$ and $\mathbf{x}_{\sim i j}$ being the $\mathbf{x}$ vector respectively without the elements $x_{i}$ and $x_{i j}$. Squaring (4.52), integrating over $K^{n}$ and considering the orthogonality, terms are obtained that 


\section{Reliability evaluation}

are identical with the variance decomposition (4.47), compare (4.48) and (4.56):

$$
\begin{aligned}
& \int_{K^{n}} g^{2}(\mathbf{x}) \mathrm{d} \mathbf{x}-g_{0}^{2} \quad=\operatorname{Var}[g(\mathbf{x})] \quad \equiv V \\
& \int_{K^{n}} g_{i}^{2}\left(x_{i}\right) \mathrm{d} x_{i} \quad \equiv \int_{0}^{1} g_{i}^{2}\left(x_{i}\right) \mathrm{d} x_{i} \quad=\operatorname{Var}\left[g_{i}\left(x_{i}\right)\right] \quad \equiv V_{i} \\
& \int_{K^{n}} g_{i j}^{2}\left(x_{i}, x_{j}\right) \mathrm{d} x_{i} \mathrm{~d} x_{j} \equiv \iint_{0}^{1} g_{i j}^{2}\left(x_{i}, x_{j}\right) \mathrm{d} x_{i} \mathrm{~d} x_{j}=\operatorname{Var}\left[g_{i j}\left(x_{i}, x_{j}\right)\right] \equiv V_{i j} \quad .
\end{aligned}
$$

These formulae, together with (4.56), allows the terms of the variance decomposition (4.48), and thus the sensitivity indices (4.50), to be evaluated by Monte Carlo. The generic first order term can be written as:

$$
\begin{aligned}
V_{i} & =\int_{0}^{1}\left[\int_{K^{n-1}} g(\mathbf{x}) \mathrm{d} \mathbf{x}_{\sim i}-g_{0}\right]^{2} \mathrm{~d} x_{i} \\
& =g_{0}^{2}-2 g_{0} \int_{K^{n}} g(\mathbf{x}) \mathrm{d} \mathbf{x}+\int_{0}^{1}\left[\int_{K^{n-1}} g(\mathbf{x}) \mathrm{d} \mathbf{x}_{\sim i} \cdot \int_{K^{n-1}} g(\mathbf{x}) \mathrm{d} \mathbf{x}_{\sim i}\right] \mathrm{d} x_{i} \\
& =-g_{0}^{2}+\int_{0}^{1} \int_{K^{n-1}} g\left(\mathbf{x}_{\sim i}^{(1)}, x_{i}\right) g\left(\mathbf{x}_{\sim i}^{(2)}, x_{i}\right) \mathrm{d} \mathbf{x}_{\sim i}^{(1)} \mathrm{d} \mathbf{x}_{\sim i}^{(2)} \mathrm{d} x_{i},
\end{aligned}
$$

where $\mathbf{x}^{(1)}$ and $\mathbf{x}^{(2)}$ denotes two different mute variables. Similarly, for the second order terms, it is:

$$
V_{i j}=\int_{0}^{1} \int_{0}^{1} \int_{K^{n-1}} g\left(\mathbf{x}_{\sim\{i j\}}^{(1)}, x_{i}, x_{j}\right) g\left(\mathbf{x}_{\sim\{i j\}}^{(2)}, x_{i}, x_{j}\right) \mathrm{d} \mathbf{x}^{(1)} \mathrm{d} \mathbf{x}^{(2)} \mathrm{d} x_{i} \mathrm{~d} x_{j}-g_{0}^{2}-V_{i}-V_{j} .
$$

Monte Carlo estimates for such expressions can be immediately defined as:

$$
\begin{aligned}
\hat{g_{0}} & =\frac{1}{N} \sum_{m=1}^{N} g\left(\mathbf{x}_{m}\right) \\
\hat{V} & =\frac{1}{N} \sum_{m=1}^{N} g^{2}\left(\mathbf{x}_{m}\right)-g_{0}^{2} \\
\hat{V}_{i} & =\frac{1}{N} \sum_{m=1}^{N} g\left(\mathbf{x}_{\sim i m}^{(1)}, x_{i m}^{(1)}\right) g\left(\mathbf{x}_{\sim i m}^{(2)}, x_{i m}^{(1)}\right)-g_{0}^{2} \\
\hat{V}_{i j} & =\frac{1}{N} \sum_{m=1}^{N} g\left(\mathbf{x}_{\sim\{i j\} m}^{(1)}, x_{i m}^{(1)}, x_{j m}^{(1)}\right) g\left(\mathbf{x}_{\sim\{i j\} m}^{(2)}, x_{i m}^{(1)}, x_{j m}^{(1)}\right)-g_{0}^{2}-V_{i}-V_{j}
\end{aligned}
$$

where $\mathbf{x}_{m}^{(1)}$ and $\mathbf{x}_{m}^{(2)}$ are now samples from two different sets. In (4.60d) the approximations $\hat{V}_{i}$ and $\hat{V}_{j}$ from (4.60c) are used for $V_{i}$ and $V_{j}$; however, for the value of $g_{0}$ required in $(4.60 \mathrm{c})$ and $(4.60 \mathrm{~d})$, the Monte Carlo estimate $\hat{g_{0}}$ from (4.60a) is not used because 


\subsection{Probabilistic sensitivity analysis}
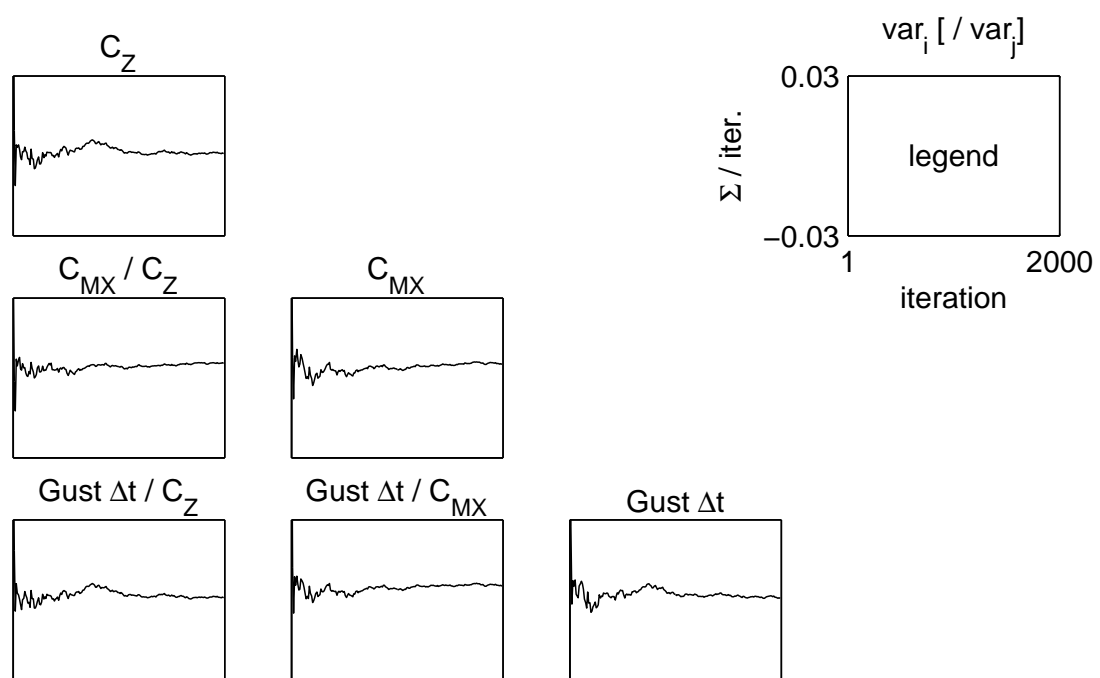

Gust $\Delta \mathrm{t} / \mathrm{C}_{\mathrm{MX}}$
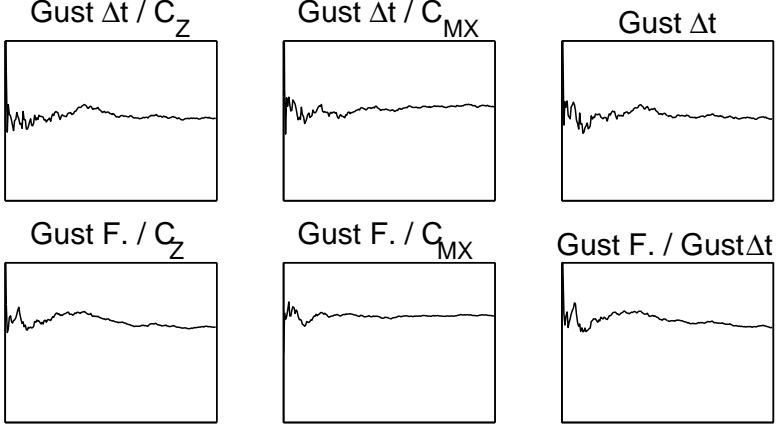

Gust F.

Figure 4.21: Convergence of the first order (main diagonal, Eq. 4.50a) and second order (offdiagonal, Eq. 4.50b) sensitivity indices (legend on the upper right corner).

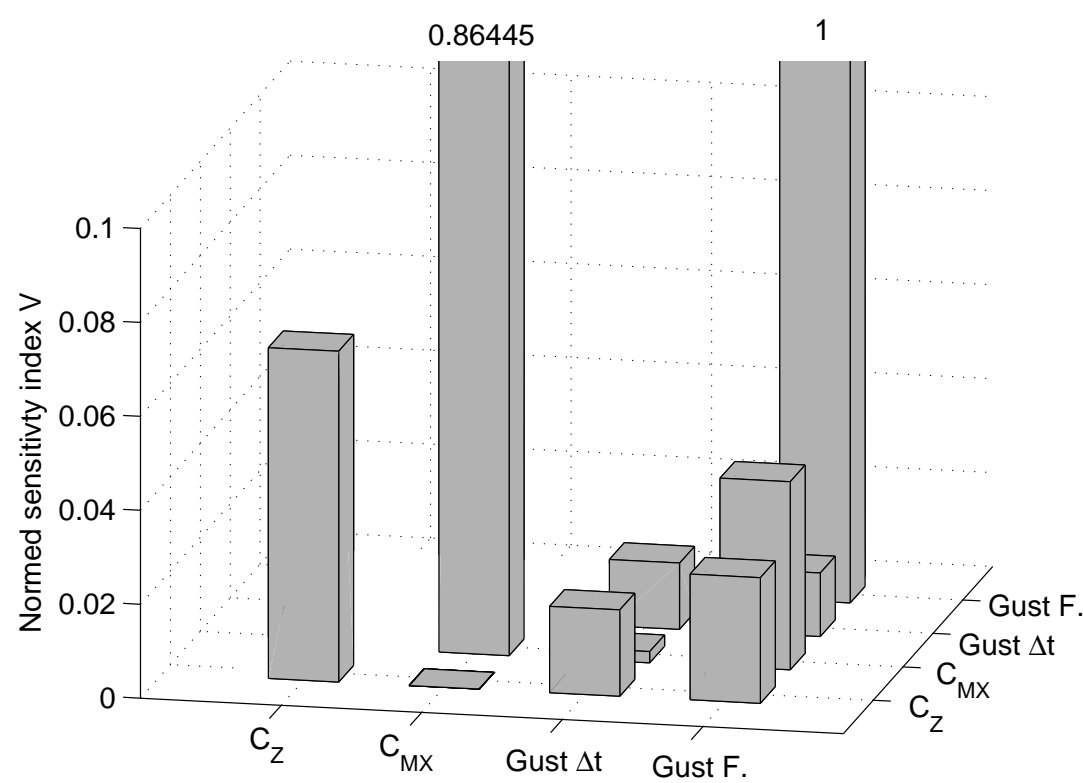

Figure 4.22: Normed first (main diagonal, Eq. 4.50a) and second order (off-diagonal, Eq. 4.50b) sensitivity indices. 


\section{Reliability evaluation}

this choice would enlarge considerably the variance of the estimates $\hat{V}_{i}$ and $\hat{V}_{i j}$. As a response surface model was used, an estimate of $g_{0}$ could be computed directly from such model by Taylor expansion.

Fig. 4.21 shows the convergence history of the Monte Carlo estimates; the final results (normalised) are plotted in Fig. 4.22. Besides the very large values of the main effects of roll moment coefficient and gust factor, which was to be expected considering the results of the sensitivity studies of the previous sections, the interaction effects between the variables can be observed. For example, the interaction between roll moment and lift coefficients is almost zero, showing that the uncertainties in these parameters propagate independently from each other. This is to say, the uncertainties in the wheel unloading is not additionally enlarged when both coefficients are uncertain. On the contrary, some interaction between roll moment coefficient and gust factor exists, indicating that it is not convenient to have uncertainties in both parameters because the resulting uncertainty in the wheel unloading is larger than the sum of the uncertainties due to both parameters separately. Even though in the case studied in this work the interaction effects are relatively little, this kind of information is in general useful for design tasks, model definition and norm compilation but also for the set up of experiments aiming to assess uncertainties.

\subsection{Optimisation}

\subsubsection{Optimisation and risk analysis}

Optimisation in the presence of uncertain parameters and in connection with reliability issues has been object of large investigation in the last years, [PSP93, OSR98, DC00]. However, an optimisation task aiming to improve exclusively the crosswind stability is not interesting because the effects of every modification on other performances would be neglected. On the contrary, of primary interest are the improvement of the crosswind stability together with other vehicle performances (multiobjective optimization) or the consideration of the crosswind stability as a boundary condition within a generic optimisation task (reliability based optimization).

In practice, because of wheel and rail wearing, derailment safety, track shift etc., a typical design goal is the minimisation of the lateral wheel force $(Y)$. Thus, the wheel force of the leading wheelset has been chosen in this work as an examplary performance for which an optimum is searched. This choice does not influence the generality of the methodology: other criteria, such as comfort or hunting stability, could be added to the problem without any modification of the formulation.

The mass of the leading bogie and the longitudinal position of the centre of gravity of the carbody have been taken as design parameters, i.e. as tuners of the optimisation; stiffness and damping coefficients could also have been used. Such deterministic parameters are denoted as $\overline{\mathbf{x}}$, whereas $\mathbf{X}$ are the stochastic parameters considered in the previous sections, see Table 4.2. Conventional SQP techniques as implemented in MOPS, an optimisation package developed at DLR, [ $\left.\mathrm{J}^{+} 02\right]$, have been used. 


\subsection{Optimisation}

Table 4.2: Parameters in the optimisation task.

\begin{tabular}{c|c||c}
\multicolumn{2}{c||}{ parameter } & function \\
\hline \hline $\mathbf{\mathbf { x }}=\left[\begin{array}{c}m_{b} \\
c_{g x}\end{array}\right]$ & $\begin{array}{c}\text { leading bogie mass } \\
\text { longit. position of } \\
\text { carbody's c.o.g. }\end{array}$ & tuners \\
\hline $\mathbf{X}$ & see Sec. 3 & $\begin{array}{c}\text { uncertain } \\
\text { parameters }\end{array}$
\end{tabular}

\subsubsection{Multiobjective optimisation}

The typical multiobjective optimisation task for the scenario defined above is to minimise the maximal wheel unloading $Q^{\prime}$ and the peak value of the lateral wheel force $Y$ due to the gust load. In the deterministic case, when only deterministic design parameters $\overline{\mathbf{x}}$ are considered, the task is formulated in the traditional way:

$$
\begin{aligned}
& \min _{\overline{\mathbf{x}}} Q^{\prime}(\overline{\mathbf{x}}) \\
& \min _{\overline{\mathbf{x}}} Y(\overline{\mathbf{x}}) \\
& \mathbf{c}(\overline{\mathbf{x}})<0,
\end{aligned}
$$

where $\mathbf{c}$ denotes the constraints.

If also stochastic parameters $\mathbf{X}$ are considered, $Q^{\prime}, Y$ and $\mathbf{c}$ are function of both deterministic and stochastic parameters. A possible reformulation of (4.61) is:

$$
\begin{gathered}
p_{F}(\overline{\mathbf{x}}, \mathbf{X})<p_{0} \\
\min _{\overline{\mathbf{x}}} Y\left(\overline{\mathbf{x}}, \boldsymbol{\mu}_{\mathbf{X}}\right) \\
\mathbf{c}\left(\overline{\mathbf{x}}, \boldsymbol{\mu}_{\mathbf{X}}\right)<0
\end{gathered}
$$

$p_{0}$ being a given upper limit for the probability of failure, e.g. $10^{-3}$. The objective for the wheel unloading has been substituted by the corresponding objective for the probability of failure. Furthermore, the lateral force $Y$ and the constraints c are evaluated at the expected values of the stochastic parameters $\mathbf{X}$, which is equivalent to a first order approximation.

The task (4.62) was applied to the test case for $U_{0}=18 \mathrm{~m} / \mathrm{s}$; for such task only compromise Pareto solutions can be found because the criteria are definitely conflicting. A part of the resulting Pareto set is reported in Fig. 4.23. The nominal value of the criteria, i.e. the values corresponding to the unmodified vehicle, are $p_{F}=0.35$ and 


\section{Reliability evaluation}

$Y=40775 \mathrm{~N}$. This value of the probability of failure is very high but, as already discussed, it is not equivalent to the risk, see Sec. 2.1.3. As to be expected, the probability of failure can only be reduced significantly for high values of the tuners and accepting increased lateral wheel forces. However, the $Y$ forces are increased of less than 10\%, whereas the probability of failure is reduced of up to a factor 3. Finally, it can be observed the Pareto set covers a little range of the lateral force $Y$; this is due to the fact that only straight track was considered.
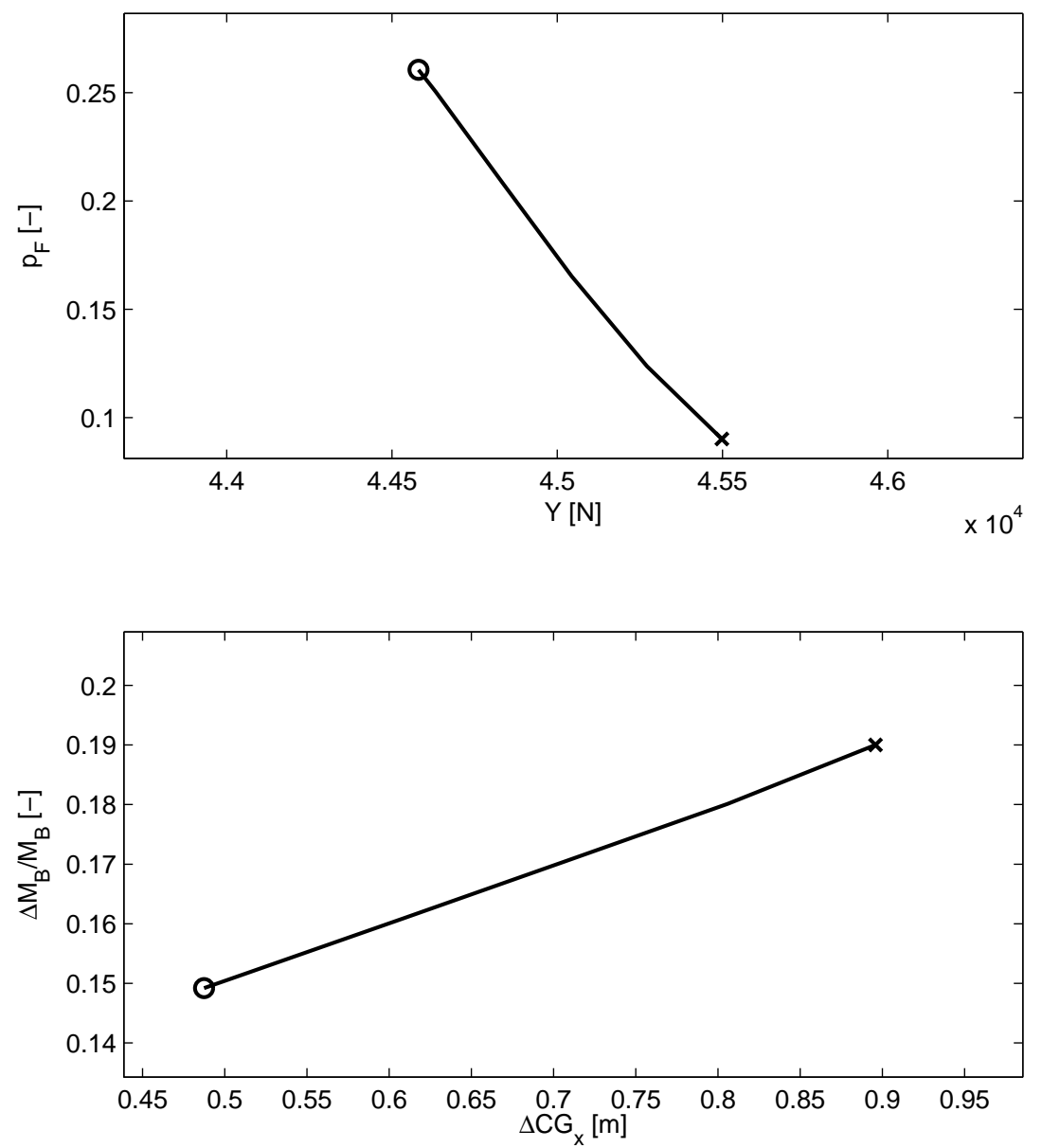

Figure 4.23: Part of the Pareto set resulting from the optimisation task (4.62): criteria (top) and tuners (bottom). $Y$ denotes the lateral wheel force of the leading wheelset, $\Delta m b / m b$ the relative change in the leading bogie's mass and $\Delta c g x$ the longitudinal displacement of the carbody's centre of gravity to the fore.

\subsubsection{Reliability based optimisation}

Reliability and crosswind stability can be considered within a generic optimisation task which does not explicitly aim to improve the crosswind stability. Consider, by way of example, a task aiming to reduce only the peak value of the lateral wheel forces $Y$. The 


\subsection{Optimisation}

conventional deterministic formulation would be:

$$
\begin{aligned}
& \min _{\overline{\mathbf{x}}} Y(\overline{\mathbf{x}}) \\
& \mathbf{c}(\overline{\mathbf{x}})<0 .
\end{aligned}
$$

The limit for the wheel unloading $\left(Q^{\prime}<Q_{L}\right)$ is included in the generic constraints $\mathbf{c}$. If also stochastic parameters $\mathbf{X}$ additionally to the deterministic ones are taken into account the criterion $Y$ as well as the constraints $\mathbf{c}$ become functions of both groups of parameters. In this case the optimisation task can be reformulated as the minimisation of the expected value of $Y$, imposing that the probability of a constraints violation is lower than a fixed level $p_{0}{ }^{5}$ :

$$
\begin{aligned}
& \min _{\overline{\mathbf{x}}} \mathrm{E}[Y(\overline{\mathbf{x}}, \mathbf{X})] \\
& \operatorname{Pr}\{\mathbf{c}(\overline{\mathbf{x}}, \mathbf{X})>0\}<p_{0} .
\end{aligned}
$$

This formulation of the constraints (4.64b) can be directly interpreted as a reliability task and the problem can be correspondingly rewritten as:

$$
\begin{aligned}
& \min _{\overline{\mathbf{x}}} Y\left(\overline{\mathbf{x}}, \boldsymbol{\mu}_{\mathbf{X}}\right) \\
& p_{F}(\overline{\mathbf{x}}, \mathbf{X})<p_{0} .
\end{aligned}
$$

This formulation is similar to (4.62). However, the probability of failure $p_{F}$ in $(4.65 \mathrm{~b})$ differs from that in (4.62a): it does not only include the probability of failure due to wheel unloading but the probability of failure connected to every other limit imposed by the constraints to the system parameters. As in the test case only the unloading limit have been considered in the constraints c, the results of the optimisation task (4.65) for different values of $p_{0}$ are identical to the Pareto set in Fig. 4.23.

\subsubsection{Robust crosswind stability}

For the sake of completeness, a third approach to optimisation in the presence of uncertain parameters is reported because of its large diffusion; however, it was not tested in this work. In the robust case the aim of the optimisation task is to minimise the expected value of the wheel unloading due to crosswind and the variations of the optimum caused by the uncertainties:

$$
\begin{aligned}
& \min _{\overline{\mathbf{x}}} \mathrm{E}\left[Q^{\prime}(\overline{\mathbf{x}}, \mathbf{X})\right] \simeq Q^{\prime}\left(\overline{\mathbf{x}}, \boldsymbol{\mu}_{\mathbf{X}}\right) \\
& \min _{\overline{\mathbf{x}}} \operatorname{Var}\left[Q^{\prime}(\overline{\mathbf{x}}, \mathbf{X})\right]
\end{aligned}
$$

The expected value and the variance obvioulsy refer to the stochastic parameters $\mathbf{X}$. The optimal configuration $\overline{\mathbf{x}}_{\text {min }}$ resulting from the task (4.66a) does not necessarily

\footnotetext{
${ }^{5}$ The stochastic parameters $\mathbf{X}$ are supposed to be unbounded. Otherwise the constraints (4.63b) could still be satisfied.
} 


\section{Reliability evaluation}

correspond to a global minimum of the unloading $Q^{\prime}$ but to a minimum which is as little influenced as possible by the variation of the parameters $\mathbf{X}$. On the contrary, a solution which is optimal for $\boldsymbol{\mu}_{\mathbf{X}}$, but badly deteriorates for other values of $\mathbf{X}$, is rejected.

The idea behind this approach is that if a design were possible in which the uncertainties do not propagate, safety limits would not be necessary. This formulation of the optimisation task can be managed again with tools from the reliability analysis. However, it makes high demands to the optimisation algorithm and tailored solutions are usually necessary. 


\section{Results}

\section{Chapter overview}

In this section selected results are presented. The most important ones are the probabilistic characteristic wind curves (PCWC) which report the probability of failure as a function of the mean wind velocity and the driving velocity: $p_{F}\left(U_{0}, V\right)$. They were computed with the FORM method and cover the assumed operative range $160 \div 280 \mathrm{~km} / \mathrm{h}$. The PCWCs are the final result of the risk analysis and can then be used within the risk assessment for the quantification of the risk as discussed in Sec. 2.1.3. It must be observed that for curved track the cant deficiency $c_{d}$ has to be considered too: $p_{F}\left(U_{0}, V, c_{d}\right)$. It follows that In this case the PCWCs cannot be as easily depicted, e.g. as surfaces, as in the straight track case.

\subsection{Straight track}

As the straight track case has been used for the exemplification of the reliability techniques, most results were already presented and discussed in Sec. 4. Here only the probabilistic characteristic wind curves and the sensitivity over the whole driving velocity range are reported.

\subsubsection{Probabilistic characteristic wind curve}

The probabilistic characteristic wind curve is reported as a surface and as set of contours in Fig. 5.1. In both cases the corresponding deterministic characteristic wind curve $\left(Q_{L}^{\prime}=0.9\right)$ is also marked. The surface is quite regular, confirming the almost linear system behaviour already observed for a single driving velocity. As to be expected, the deterministic CWC lies in a range of low probability of failure $\left(p_{F} \simeq 0.1\right)$. However, it is not exactly identical with a contour of the PCWC; it would if the system were perfectly linear.

\subsubsection{Sensitivity}

In Fig. 5.2 the sensitivity of the probability of failure with respect to mean and standard deviation of the aerodynamic roll moment coefficient and the gust factor is reported. These two parameters were proven to be the most influential, see Sec. 4.3 and Fig. 4.19 on page 78 . 


\section{Results}
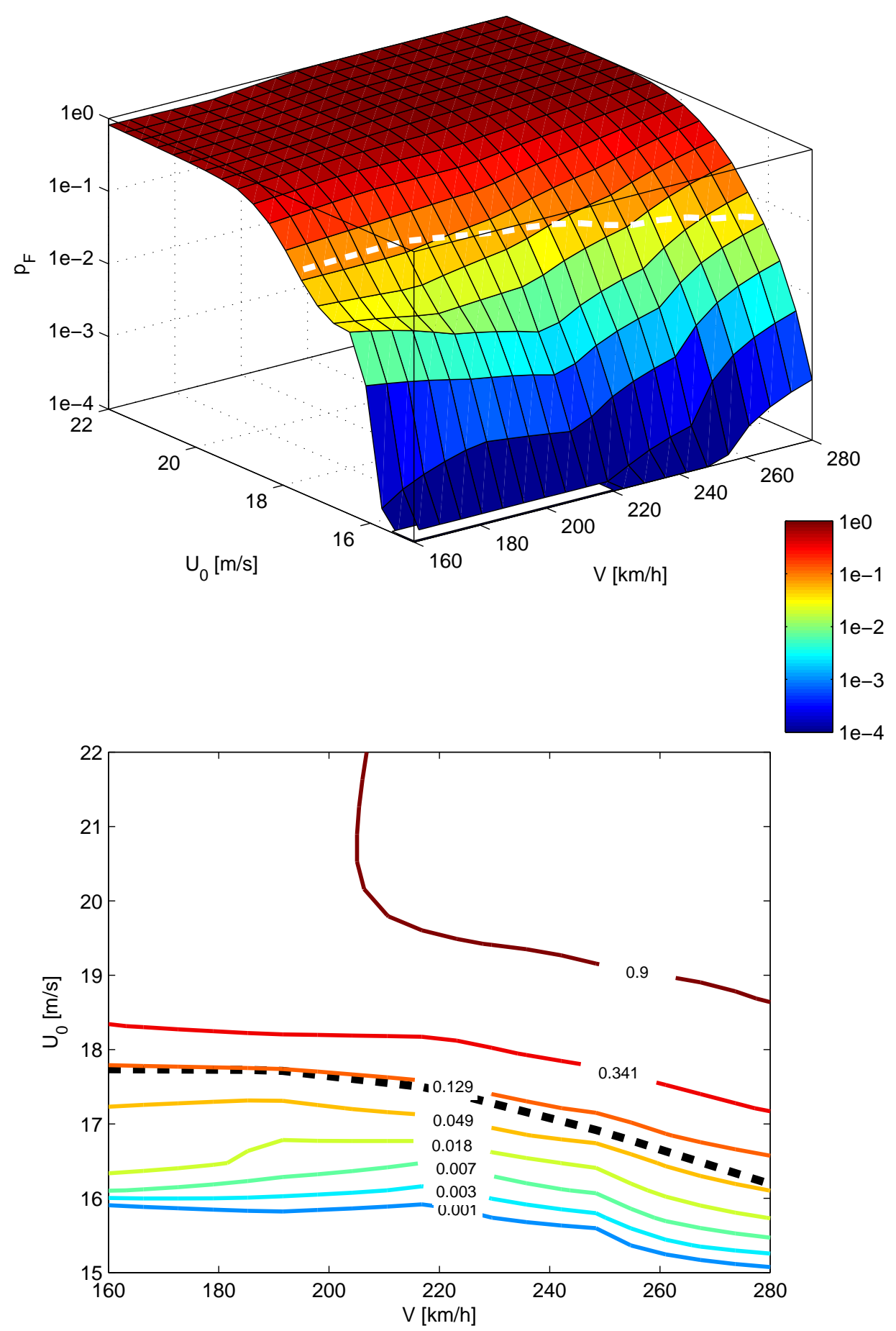

Figure 5.1: Probabilistic Characteristic Wind Curve for the straight track case. The dashed line is the deterministic $\mathrm{CWC}\left(Q_{L}^{\prime}=0.9\right)$ and lies in a range $p_{F} \in(0.071 ; 0.159)$. 


\subsection{Straight track}
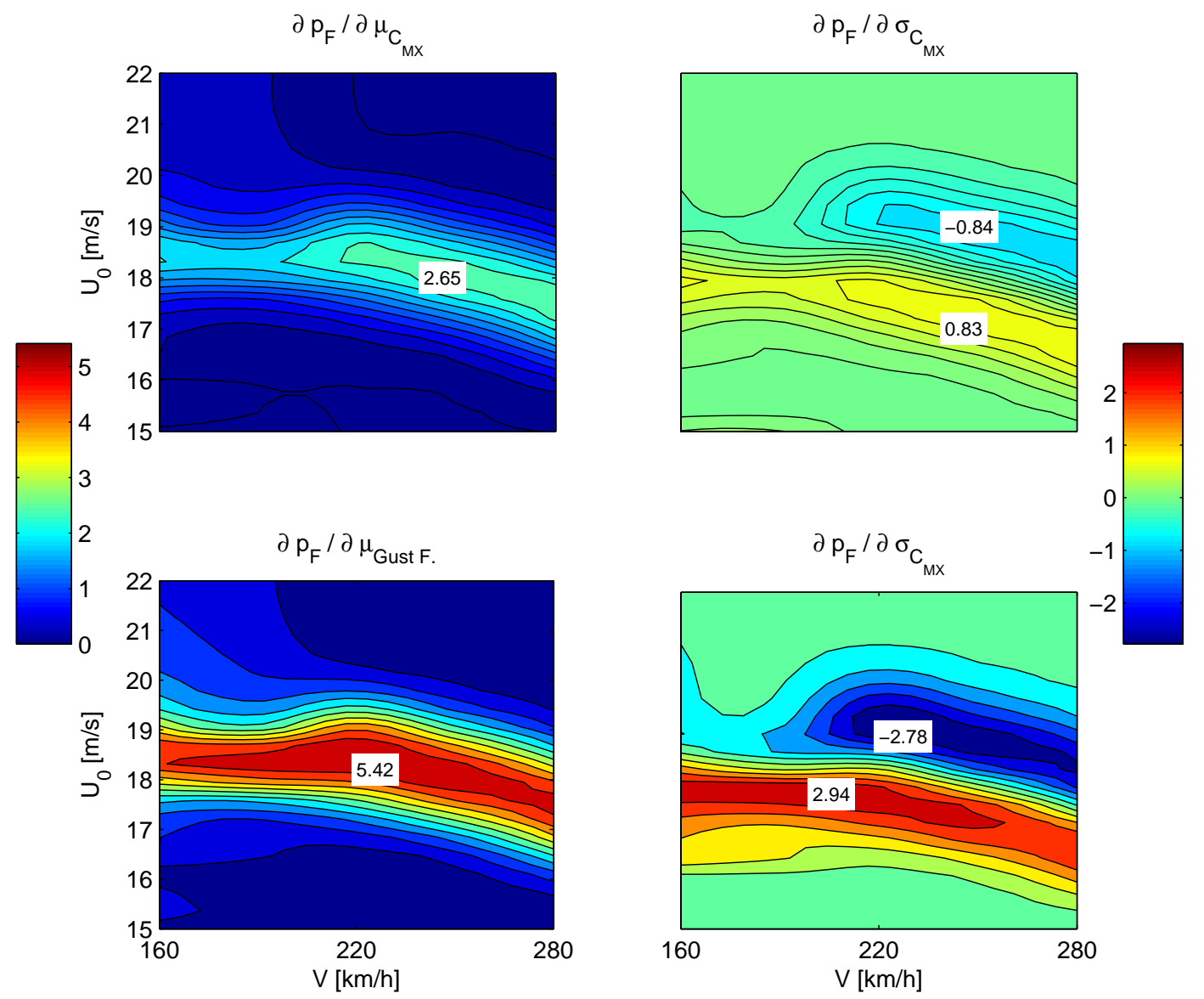

Figure 5.2: Sensitivity of the probability of failure with respect to the mean (left column) and standard deviation (right column) of the aerodynamic roll moment coefficient (top row) and the gust factor (bottom row). Compare with Fig. 4.19 on page 78.

As to the mean of the variables (Fig. 5.2, left column), it can be observed that the sensitivity is slightly larger for higher than for lower driving velocities. This means that, for both parameters, inaccuracy in the estimation of the mean values has larger influence on the evaluation of the probability of failure. On the other hand, it also means that an increase/decrease of the roll moment coefficient by design modifications leads to larger deterioration/improvement of the probability of failure.

As to the variance (Fig. 5.2, right column), the typical pattern of the derivative, explained in Sec. 4.3, must be borne in mind; the most important point was that the derivative is zero for $p_{F}=0.5$. The diagrams show that for lower driving velocities the sensitivity is large for $p_{F}<0.5$ (low values of $U_{0}$ ) and rather low for $p_{F}>0.5$ (high values of $U_{0}$ ), whereas in the middle-high driving velocity range the situation is inverted and the sensitivity has a prominent (negative) peak for $p_{F}>0.5$. However, as the region of low probability of failure is in practice the most decisive, the latter peak is not very important. It can be concluded that the sensitivity with respect to the variance is almost uniform over the driving velocity range, lower velocities being slightly more sensitive. 


\section{Results}

\subsubsection{Optimisation}

In Fig. 5.3 the PCWC for the optimised vehicle is reported. From the Pareto set the configuration that minimises the probability of failure was chosen, cp. Fig. 4.23 on page 88 . It can be noted that for $V=250 \mathrm{~km} / \mathrm{h}$, which was the optimisation scenario, the deterministic CWC gains only about $5 \%$ whereas the probability of failure is approximatively halved.

\subsection{Curve}

In this section results for curved track are reported. Some general results are firstly plotted, which also allow a qualitative comparison with the behaviour of the vehicle on straight track, followed by some characteristic wind curves. As already mentioned, in this case the probabilistic characteristic wind curve is also a function of the cant deficiency: $P C W C\left(U_{0}, V, c_{d}\right)$. In the simulations the cant was fixed and the curve radius changed; in Table 5.1 the curve radii for the studied ranges of driving velocity and cant deficiency are reported assuming a cant $c=0.18 \mathrm{~m}$, cp. Eq. (3.1) on page 46. For non tilting trains the unbalanced lateral acceleration $a_{l}=1 \mathrm{~m} / \mathrm{s}^{2}$ is usually considered as worst case; nonetheless, the very high value $a_{l}=1.31 \mathrm{~m} / \mathrm{s}^{2}$ corresponding to a cant deficiency $c_{d}=0.2 \mathrm{~m}$ has also been considered in this work for reference.

Sensitivity results for curved track are not presented because for fixed driving velocity (i.e. $\left.\partial P_{F}\left(U_{0}, c_{d}\right) / \partial *\right)$ the system behaviour is almost linear, as shown below, whereas for fixed cant deficiency (i.e. $\left.\partial P_{F}\left(U_{0}, V\right) / \partial *\right)$ the results are very close to those of the straight track case.

Table 5.1: Curve radii for the studied cant deficiencies and driving velocities setting the cant $c=0.18 \mathrm{~m}$, cp. Eq. (3.1).

\begin{tabular}{|c|r||r|r|r|r|r|}
\hline \multicolumn{2}{|c||}{ curve } & \multicolumn{5}{c|}{ driving vel. [km/h] } \\
\cline { 3 - 7 } radius $[\mathrm{m}]$ & 160 & 190 & 220 & 250 & 280 \\
\hline \hline cant & $0.00 ; 0.00$ & 1679 & 2368 & 3175 & 4100 & 5144 \\
deficiency $[\mathrm{m}]$ & $0.05 ; 0.33$ & 1314 & 1853 & 2485 & 3209 & 4025 \\
$;$ & $0.10 ; 0.65$ & 1079 & 1522 & 2041 & 2636 & 3306 \\
unbalanced & $0.15 ; 0.98$ & 916 & 1291 & 1732 & 2236 & 2805 \\
lat. acc. $\left[\mathrm{m} / \mathrm{s}^{2}\right]$ & $0.20 ; 1.31$ & 795 & 1121 & 1504 & 1942 & 2436 \\
\hline
\end{tabular}

\subsubsection{General results}

In Fig. 5.4 the Most Probable Point for curved track with $V=250 \mathrm{~km} / \mathrm{h}, U_{0}=16 \mathrm{~m} / \mathrm{s}$ and $c_{d}=0 \mathrm{~m}$ is reported. If compared with the corresponding plot for the straight track case, Fig. 4.6 on page 62, it can be observed that the behaviour of the system on straight and curved track are very similar. The MPP loci, not reported, are very similar too. 


\subsection{Curve}
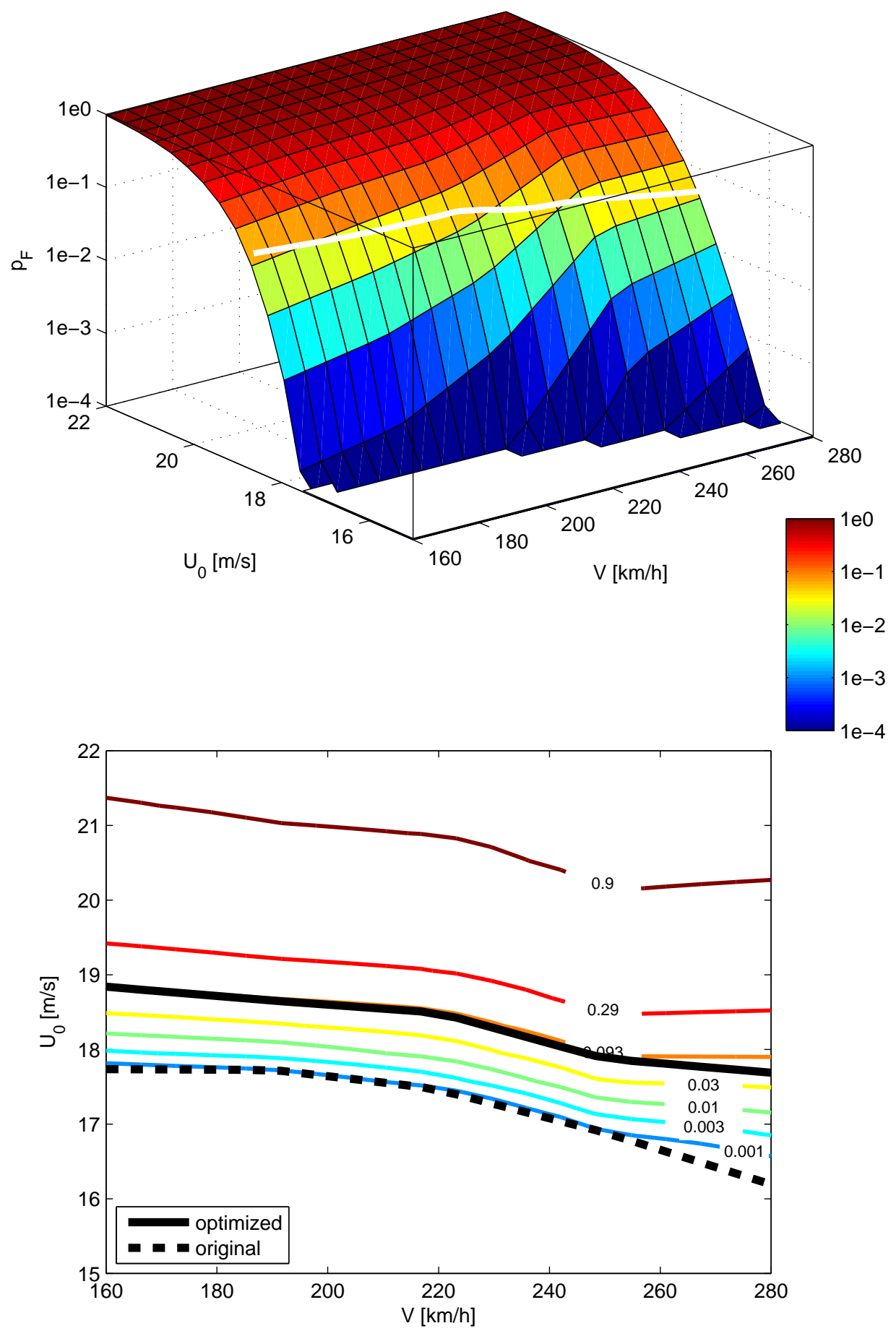

Figure 5.3: Probabilistic characteristic wind curve for the optimised vehicle, straight track case. The thick dashed line is the deterministic CWC $\left(Q_{L}^{\prime}=0.9\right)$. The deterministic CWC of the original vehicle (thick continuous line) is also reported for reference. 


\section{Results}
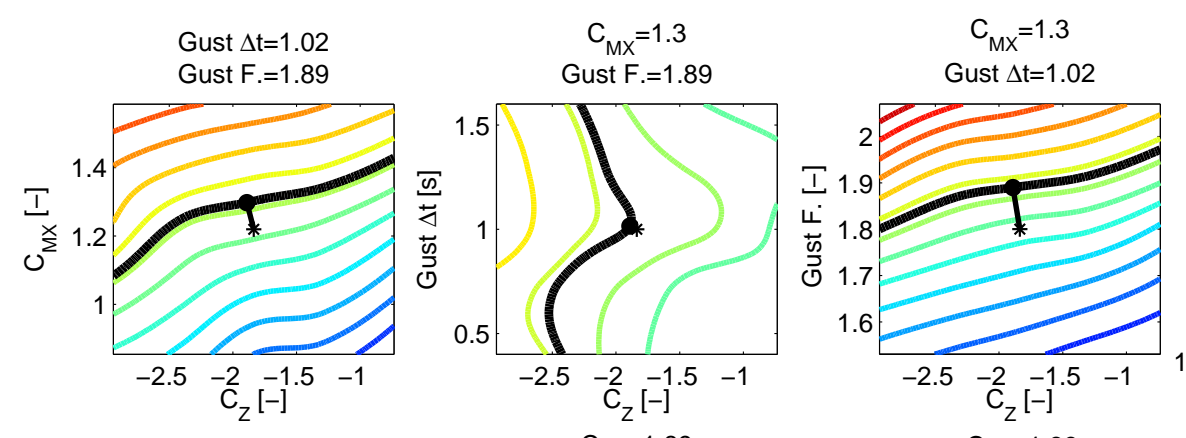

$$
C_{z}=-1.89
$$

$C_{z}=-1.89$
Gust F.=1.89

Gust $\Delta \mathrm{t}=1.02$
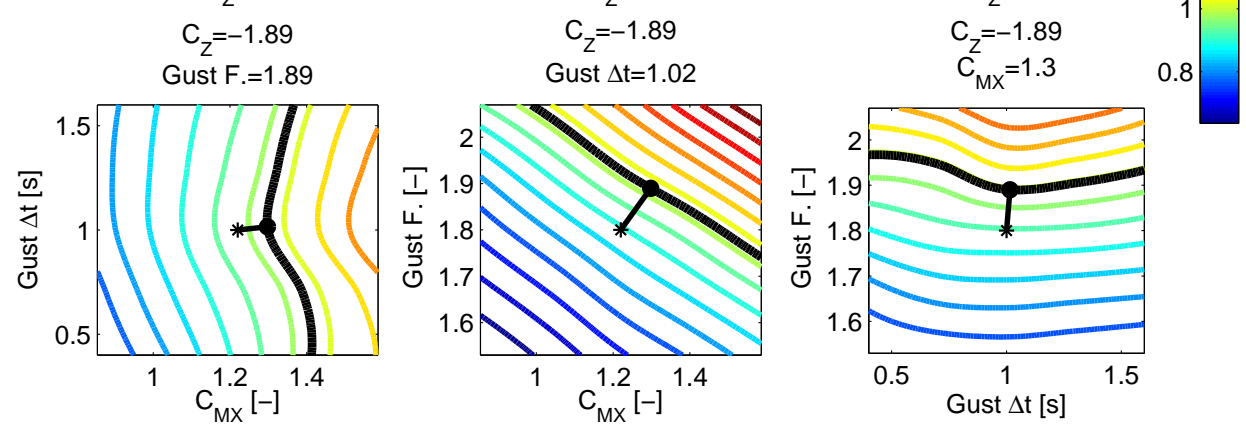

Figure 5.4: MPP with contours of the performance function for curved track with $U_{0}=16 \mathrm{~m} / \mathrm{s}$, $c_{d}=0 \mathrm{~m}, V=250 \mathrm{~km} / \mathrm{h}$. The thick line denotes the limit state $g(x)=0$, i.e. $Q^{\prime}=1$. See also comment of Fig. 4.6 on page 62.

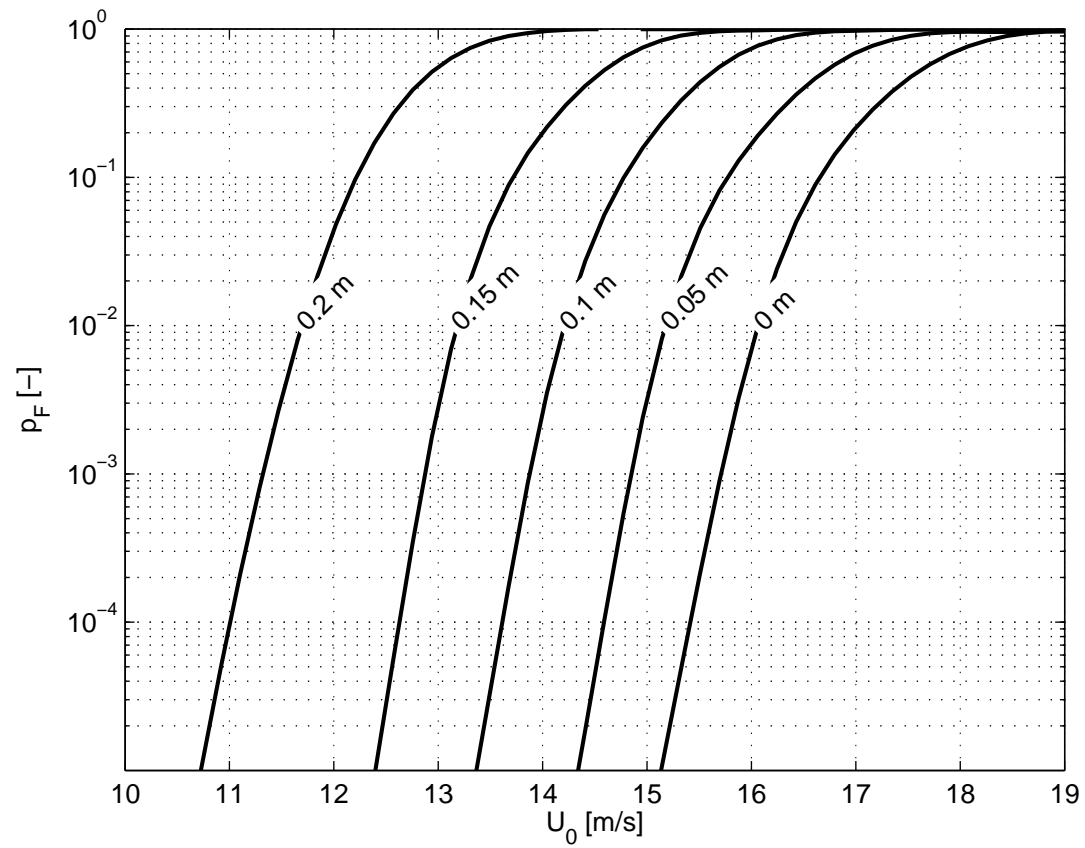

Figure 5.5: Probability of failure as a function of $U_{0}$ parameterised with the cant deficiency for $V=250 \mathrm{~km} / \mathrm{h}$. 
Fig. 5.5 reports the probability of failure as a function of the mean wind velocity for different cant deficiencies. For $c_{d}=0 \mathrm{~m}$ the curve is very close to the corresponding curve on straight track, cp. Fig. 4.8 on page 64 , but the huge influence of cant deficiency is pointed out. For example, for $c_{d}=0.2 \mathrm{~m}$ the stability is deteriorated of $5 \mathrm{~m} / \mathrm{s}$.

\subsubsection{Characteristic wind curves}

In Fig. 5.6 the deterministic characteristic wind curves (overturning wind velocity over cant deficiency) for curved track are plotted for different driving velocities, having assumed an unloading limit $Q_{L}^{\prime}=0.9$. The curves display an almost regular pattern which suggests that nonlinearities are not very influential. Only for the highest velocity taken into account $(280 \mathrm{~km} / \mathrm{h})$ and large cant deficiency the stability performance of the vehicle seems to be particularly bad.

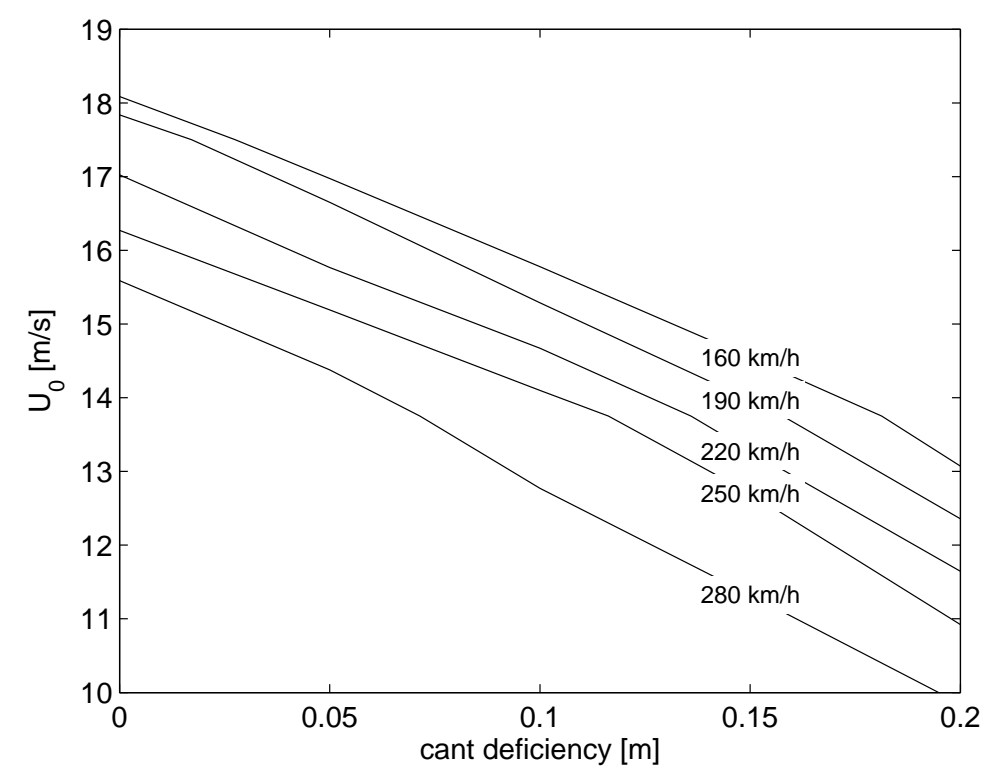

Figure 5.6: Deterministic CWC for curved track $\left(Q_{L}^{\prime}=0.9\right)$.

As to the probabilistic characteristic wind curves, it was already noted that, for curved track, it cannot be easily plotted, as it is a function of three parameters. By way of example, the PCWC corresponding to $V=250 \mathrm{~km} / \mathrm{h}$, i.e. $p_{F}\left(U_{0}, 250, c_{d}\right)$, is reported in Fig. 5.7 as a surface and contours; the deterministic CWC is also plotted for comparison. Except for the kink at high values of the cant deficiency, the PCWC shows a very regular pattern which is representative also for the driving velocities not reported here. 


\section{Results}
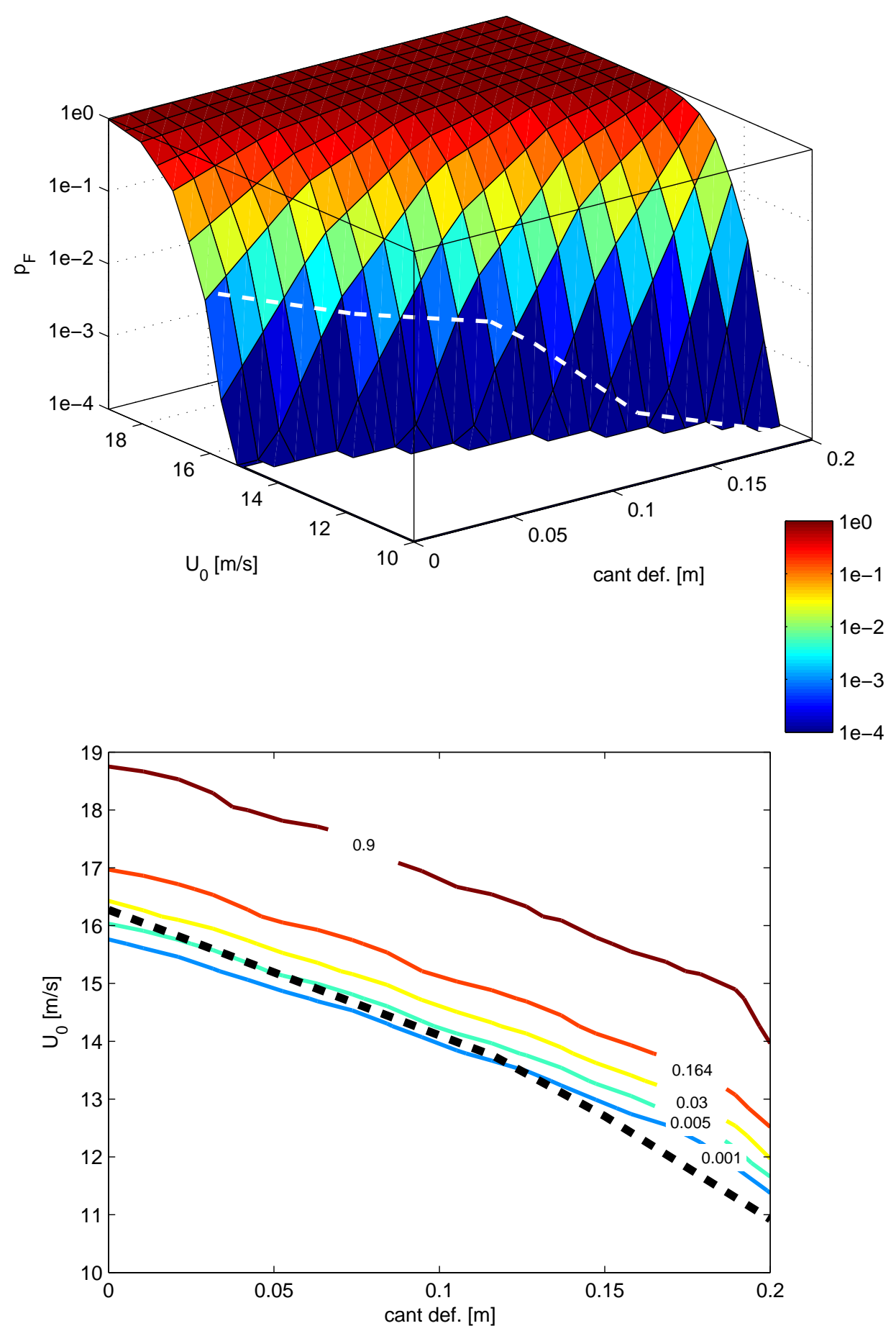

Figure 5.7: Probabilistic characteristic wind curve (PCWC) for $V=250 \mathrm{~km} / \mathrm{h}$. The thick dashed line is the deterministic $\mathrm{CWC}\left(Q_{L}^{\prime}=0.9\right)$. 


\section{Summary and conclusions}

\subsection{Main results and contributions}

The common approach to the crosswind stability analysis of railway vehicles is based on the assumption that all parameters of the global system, composed of vehicle and environment, are exactly known and constant. This is a poor modelling of reality as some of the parameters are known to be affected by large uncertainty and/or variability; the main consequences of this choice are the necessity for over-conservative safety limits and an inefficient safety check. But, as the crosswind stability of railway vehicles is very difficult to improve, the accuracy in the risk evaluation should be as high as possible.

To overcome such limitations, a reliability based approach to the risk analysis, influencing also the risk assessment, has been presented, discussed and applied to the real case of a modern vehicle. Different modelling options and computational techniques have been introduced, showing that the level of accuracy can be adapted to the available inputs, i.e. the statistical description of the parameters, and the planned computational effort. A compromise which is computationally bearable and leads to high accuracy was used to compute the final results.

The advantages of the proposed approach are:

- The uncertainties have not to be compensated by empirical safety limits but their actual contribution to the risk is efficiently managed.

- A closer to reality risk evaluation is achieved. This means that at the very end of the risk assessment process it is not simply known if the system is safe or not but a value for the risk is produced, i.e. the degree of safety of the system is quantified.

- A general deeper insight into the behaviour of the system is allowed because the whole range of the system parameters is considered and studied, whereas in the conventional approach only the limit between safe and unsafe region is taken into account.

A secondary objective reached by this work is the suggestion of possible improvements in the modelling. With regard to the aerodynamics, the influence of unsteady effects and their possible modelling by admittance functions were discussed; moreover, the possible substitution of the ideal gust with real measured wind data has been addressed. On the driving dynamics side, the adequacy of multibody simulations neglecting the wheel/rail contact was shown, which would eventually lead, with the related advantages, to linear models. 


\section{Summary and conclusions}

\subsection{Suggestions}

The proposed approach is based on the availability of a statistical description of the uncertainty/variability in the parameters. For the parameters defining the wind scenario good models are already available from the field of building aerodynamics and, mainly in the context of wind energy plants, new milestones were recently achieved. On the other hand, the aerodynamic coefficients of the vehicles are affected by a dramatic lack of knowledge: not only are they known with poor accuracy, which is the motivation of this work, but also information on their fluctuations, or even on simple bounds, is very scarce.

To this aim a lot of work is still to be done by aerodynamicists. Large benchmark studies should be performed on the example of $\left[\mathrm{B}^{+} 04\right]$ and, which is more important, systematical comparisons between results from different wind tunnels should be carried out. Finally, full scale tests on driving vehicles, even though expensive and complex, should provide basic information on the phenomena occurring during real operation. In this context a previous sensitivity analysis could give valuable information to rank the uncertainties having the most influence on the risk evaluation, and thus to direct the experimental efforts.

With regard to the improvement of the crosswind stability - not considering aerodynamic devices and/or special aerodynamic design of the bow - few effective solutions are known. One of the most discussed debatable points is the interaction between vehicle and instantaneous environmental conditions: in some countries, like Japan and France, alert systems based on anemometers are used, whereas in others, like Germany, the wind velocity encountered by the vehicle is not taken into account. When an alert system is used, the reaction to gale winds is always to reduce the driving velocity, but actually also other kinds of intervention could be conceived.

For example, the position of the centre of gravity of the vehicle is known to have a large effect on the crosswind stability, top-heaviness corresponding to larger stability. On the other hand, this choice is not favourable for the driving dynamics because it enlarges, for example, the lateral forces at the wheel/rail interface. A solution could be to displace the centre of gravity towards the front when gale winds are reported, which could be realised by shifting heavy underbelly equipment like the transformer. Worse driving dynamic properties would have to be accepted for a short time but a reduction of driving velocity could be avoided. Similar kinds of automatic modification of the vehicle configuration are already implemented in practice; in the Swiss ICN, for example, the pantograph is laterally shifted according to the tilting in the curve.

Alternatively, an improvement of the crosswind stability could be achieved by semiactive secondary suspension. As the swaying motion of the carbody is responsible for a relevant part of the wheel unloading, a semi-active secondary suspension could be used to reduce - or even advantageously influence - such motion in the presence of strong winds. Once again, a reduction in passengers' comfort and vehicle performance should be borne only for limited periods. However, the use of semi-active devices of any kind aiming to enlarge the vehicle's operative range is problematic because safety must be guaranteed also in the case of a system failure. 


\subsection{Further work}

\subsection{Further work}

The final aim of this work was to suggest a new framework for the assessment of the crosswind stability. Such a framework includes many topics which could only be mentioned and need to be deeply investigated. For example:

- The consideration of the interdependencies between mean wind velocity, gust amplitude and gust length. This will also call for a closer coalescence of risk analysis and risk assessment.

- The extraction of the statistics of the ideal gust from measured wind data.

- The use of tailored optimisation algorithms which embed the reliability formulation.

- The use of the linear mechanical model, possibly with real wind samples instead of the ideal gust.

The last item suggests a theme which should be given particular attention: the time dependent reliability. The reliability techniques used in this work presupposed a time independent system behaviour; this is very compatible with the ideal gust approach, where only the peak response is of interest. On the contrary, if real wind samples are considered, the dependency on time cannot be neglected in the reliability evaluation. However, even though time dependent reliability is a widely studied field (e.g. for fatigue related problems) satisfactory methodologies as in the time independent case are not yet available. For the crosswind stability task the first passage formulation could be used. The probability of failure in a time interval $T$ would be defined as the probability that the process $Q^{\prime}$ upcrosses the limit $Q_{L}^{\prime}$. If such upcrossing is modelled as a Poisson process with rate $\nu_{Q_{L}^{\prime}}^{+}$the probability of failure can be expressed as:

$$
p_{F}=1-\exp \left[-\int_{0}^{T} \nu_{Q_{L}^{\prime}}^{+} \mathrm{d} t\right]
$$

This apparently simple formulation calls for many assumptions and can be directly used only for simple systems, [WP095]. Nonetheless, efforts should be made in this direction because only a time dependent reliability approach will allow a definitive solid framework for the crosswind stability analysis to be established. 


\section{Bibliography}

[AHSS04] E. Andersson, J. Häggström, M. Sima, and S. Stichel. Assessment of trainoverturning risk due to strong cross-winds. J. of Rail and Rapid Transit, 218:213-223, 2004.

[AIA98] AIAA, Reston (Va,USA). Guide for the verification and validation of computational fluid dynamics simulations, 1998.

[And95] J.D. Anderson. Computational Fluid Dynamics. McGraw-Hill, New York, 1995.

[AT84] A.H-S. Ang and W.H. Tang. Probability concepts in engineering planning and design, Vol. II. Wiley \& Sons, New York, 1984.

$\left[\mathrm{B}^{+} 03\right] \quad \mathrm{M}$. Bocciolone et al. Wind tunnel tests for the identification of the aerodynamic forces on rail vehicles. In Proc. of ICWE 2003, 11th International Conference of Wind Engineering, June 2-5, Lubbock, Texas, 2003.

$\left[\mathrm{B}^{+} 04\right] \quad$ C.J. Baker et al. Measurements of the cross wind forces on trains. J. of Wind Eng. and Ind. Aerodynamics, 92:547-563, 2004.

[BAH96] R.L. Bisplinghoff, H. Ashley, and R.L. Halfman. Aeroelasticity. Dover, Mineola (N.Y.), 1996.

[Bak91a] C.J. Baker. Ground vehicles in high cross winds. Part I: Steady aerodynamic forces. J. of Fluids and Structures, 5:69-90, 1991.

[Bak91b] C.J. Baker. Ground vehicles in high cross winds. Part II: Unsteady aerodynamic forces. J. of Fluids and Structures, 5:91-111, 1991.

[Bak91c] C.J. Baker. Ground vehicles in high cross winds. Part III: The interaction of aerodynamic forces and the vehicle system. J. of Fluids and Structures, 5:221-241, 1991.

[Bak94] C.J. Baker. The quantification of accident risk for road vehicles in cross winds. J. of Wind Eng. and Ind. Aerodynamics, 52:93-107, 1994.

[BB90] C.G. Bucher and U. Bourgound. A fast and efficient response surface approach for structural reliability problems. Structural Safety, 7:57-66, 1990.

[BC02] W. Bierbooms and P.-W. Cheng. Stochastic gust model for design calculations of wind turbines. J. of Wind Eng. and Ind. Aerodynamics, 90:12371251, 2002.

[Ber87] H. Bergström. A statistical analysis of gust characteristics. Boundary latyer meteorology, 39:153-173, 1987. 


\section{Bibliography}

[BGC04] H.-R. Bae, R.V. Grandhi, and R.A. Canfield. Epistemic uncertainty quantification techniques including evidence theory for large scale structures. Computers and Structures, 82:1101-1112, 2004.

[BH96] C.J. Baker and N.D. Humphreys. Assessment of the adequacy of various wind tunnel techniques to obtain aerodynamic data for ground vehicles in cross winds. J. of Wind Eng. and Ind. Aerodynamics, 60:46-98, 1996.

[BS03] C.J. Baker and M. Sterling. Current and recent international work on railway aerodynamics. Report Issue number 4, August 11th, School of Engineering, University of Birmingham, 2003.

[Buc88] C.G. Bucher. Adaptive sampling - An interactive fast Mone Carlo procedure. Structural Safety, 5:119-126, 1988.

[Bun96a] Bundesministerium für Verkehr (German Federal Ministry of Transport), Bonn. Bewertungsverfahren für Sicherheitsfragen im Eisenbahnbetrieb, 1996.

[Bun96b] Bundesministerium für Verkehr (German Federal Ministry of Transport), Bonn. Risikoorientierte Sicherheitsnachweise im Eisenbahnbetrieb, 1996.

[CCDT03] F. Cheli, R. Corradi, G. Diana, and G. Tomasini. A numerical-experimental approach to evaluate the aerodynamic effects on rail vehicle dynamics. In Proc. of 18th IAVSD Symposium, Kanagawa, Japan, 2003. To be published as Supplement to Vehicle System Dynamics.

[CDMZ00] F. Cheli, P. Dellachá, P. Mandelli, and A. Zasso. Effects of non-steady aerodynamic forces on car handling. In Proc. of 15th European ADAMS User's Conference, November 15-17, Rome, 2000.

[CEN05] CEN (European Committee for Standardization). EN 14067-4 - Railway applications - Aerodynamics - Part 4: Requirements and test procedures for aerodynamics on open track, 2005.

[Coo79] R.K. Cooper. The probability of trains overturning in high winds. In Proc. of 5th Int. Conf. on Wind Engineering, June, Fort Collins, Colorado, pages 1185-1194, 1979.

[Coo81] R.K. Cooper. The effects of cross winds on trains. ASME J. Fluid Mech., 103:170-178, 1981.

[Coo84] R.K. Cooper. Atmospheric turbulence with respect to moving ground vehicles. J. of Wind Eng. and Ind. Aerodynamics, 217:215-238, 1984.

[Cop87] J.M. Copley. The three-dimensional flow around railway trains. J. of Wind Eng. and Ind. Aerodynamics, 26, 1987.

$\left[\mathrm{D}^{+} 03\right]$ D. Delaunay et al. Designing a wind alarm system for the TGVMéditerranée. In Proc. of ICWE 2003, 11th International Conference of Wind Engineering, June 2-5, Lubbock, Texas, 2003.

[DA88] R Dukkipati and J.R. Amyot. Computer-aided Simulation in Railway Dy- 


\section{Bibliography}

[Dav64] A.G. Davenport. Note on the distribution of the largest value of a random function with application to gust loading. Proceedings of the Institution of Civil Engineers, 28:187-195, 1964.

[DC00] X. Du and W. Chen. Towards a better understanding of modeling feasibility robustness in engineering design. ASME J. of mechanical design, 122(4):385$394,2000$.

[DEST04] B. Diedrichs, M. Ekequist, S. Stichel, and H. Tengstrand. Quasi-static modeling of wheel-rail interaction due to crosswind effects for various types of high-speed rolling stock. J. of Rail and Rapid Transit-Proc. Instn Mech. Engrs Part F, 218:133-148, 2004.

[Die03] B. Diedrichs. On computational fluid dynamics modeling of crosswind effects for high-speed rolling stock. J. of Rail and Rapid Transit-Proc. Instn Mech. Engrs Part F, 217:203-226, 2003.

[DIN05] DIN (Deutsches Institut für Normung - German institute for standardization). DIN 1055-4 Einwirkungen auf Tragwerke - Teil 4: Windlasten, 2005.

[DM96] O. Ditlevsen and H.O. Madsen. Structural Reliability Methods. Wiley \& Sons, New York, 1996.

[DR99] R.G. Dominy and A. Ryan. An improved wind tunnel configuration for the investigation of aerodynamic cross wind gust response. Paper 1999-01-0808, SAE (Society of Automotive Engineers), 1999.

[DS03] X. Du and A. Sudjianto. Reliability-based design with the mixture of random and interval variables. In Proc. of DETC'03, Sept. 2-6, Chicago, Illinois, 2003. DETC2003/DAC-48709.

[ESF98] E. Eich-Soellner and C. Führer. Numerical Methods in Multibody Dynamics. Stuttgart, Teubner, 1998.

[Fil03] A. Filippone. Unsteady gust response of road vehicles. J. of Fluids Eng., $125: 806-812,2003$.

[Gaw94] R.G. Gawthorpe. Wind effects on ground transportation. J. of Wind Eng. and Ind. Aerodynamics, 52:73-92, 1994.

[Glü85] H. Glück. Aerodynamik der Schienenfahrzeuge. TÜV Rheinland, Köln, 1985.

[HE73] W.H. Hucho and H.J. Emmelmann. Theoretical prediction of the aerodynamic derivatives of a vehicle in cross wind gusts. Paper 730232, SAE (Society of Automotive Engineers), 1973.

[HE83] J.P. Howell and K.W. Everitt. Gust response of a high speed train model. Aerodynamics of Transportation, 7:81-89, 1983.

[HM95] A. Haldar and S. Mahadevan. Probability, Reliability and Statistical Methods in Engineering Design. Wiley \& Sons, New York, 1995. 
[Hob88] F.M. Hoblit. Gust loads on aircraft. AIAA, Washington DC, 1988.

[How86] J.P. Howell. Aerodynamic response of MAGLEV train models to a crosswind gust. J. of Wind Eng. and Ind. Aerodynamics, 22:205-213, 1986.

[HS96] T. Homma and A. Saltelli. Importance measures in global sensitivity analysis of nonlinear models. Reliability Engineering and System Safety, 52:1-17, 1996.

[Huc98] W.H. Hucho, editor. Aerodynamics of Road Vehicles. SAE (Society of Automotive Engineers), Warrendale (N.Y.), 1998.

[Huc02] W.H. Hucho, editor. Aerodynamik der stumpfen Körper. Vieweg, Braunschweig (Germany), 2002.

$\left[\mathrm{I}^{+} 02\right] \quad T$. Imai et al. New train regulation method based on wind direction and velocity of natural wind against strong winds. J. of Wind Eng. and Ind. Aerodynamics, 90:1601-1610, 2002.

[Ibi53] R. Ibing. Windkanalmessungen an Triebwagenmodellen. Technical report, Institut für Eisenbahnmaschinenwesen, Technische Hochschule, Hannover, 1953.

[IEC93] IEC (International Electrotechnical Commission). IEC 61400-1 Wind Turbine Safety and Design, 1993. Adopted in Germany as DIN EN 61400-1 (2004).

$\left[\mathrm{J}^{+} 02\right] \quad$ H.D. Joos et al. A multi-objective optimization based software environment for control systems design. In Proc. of 2002 IEEE International Conference on Control Applications, September 18-20, Glasgow, U.K., 2002.

[Kar88] A. Kareem. Effect of parametric uncertainties on wind excited structural response. J. of Wind Eng. and Ind. Aerodynamics, 30:233-241, 1988.

[Kar02] G.E. Karniadakis. Quantifying uncertainty in CFD. Editorial. J. of Fluids Eng., 124:2-3, 2002.

[KL94] W. Kortüm and P. Lugner. Systemdynamik und Regelung von Fahrzeugen. Springer, Berlin Heidelberg New York, 1994.

[Kru82] H.L. Krugmann. Lauf der Schienenfahrzeuge im Gleis. Oldenbourg, München Wien, 1982.

[KS93] W. Kortüm and R.S. Sharp, editors. Multibody Computer Codes in Vehicle System Dynamics, volume 22 of Supplement to Vehicle Sytem Dynamics. Swets and Zeitlinger B.V., Amsterdam and Lisse, 1993.

[KS94] I. Krönke and H. Sockel. Model tests about cross wind effects on containers and wagons in atmosperic boundary layers. J. of Wind Eng. and Ind. Aerodynamics, 52:109-119, 1994.

[KS03] K. Knothe and S. Stichel. Schienenfahrzeugdynamik. Springer, Berlin Heidelberg New York, 2003. 


\section{Bibliography}

[KSA01] W. Kortüm, W.O. Schiehlen, and M. Arnold. Software tools - from multibody system analysis to vehicle system dynamics. In H. Aref and J.W. Phillips, editors, Mechanics for a New Millennium, pages 225-238. Kluwer Academic Publishers, Dordrecht, 2001.

[LBH03a] G.C. Larsen, W. Bierbooms, and K.S. Hansen. Mean gust shapes. Technical Report Ris $\varnothing-$ R-1133(EN), Risø National Laboratory, Roskilde, Denmark, 2003.

[LBH03b] G.C. Larsen, W. Bierbooms, and K.S. Hansen. Statistics of local extremes. Technical Report Ris $\varnothing-R-1220(\mathrm{EN})$, Ris $\varnothing$ National Laboratory, Roskilde, Denmark, 2003.

[Lin01] M. Lindahl. Track geometry for high-speed railways. Technical Report TRITA-FKT 2001:54, Royal Institute of Technology - Railway Technology, Stockholm, 2001.

[Lip99] S. Lippert. On side wind stability of trains. Technical Report TRITA-FKT 1999:38, Royal Institute of Technology - Railway Technology, Stockholm, 1999 .

[MB04] B. Möller and M. Beer. Fuzzy randomness : uncertainty in civil engineering and computational mechanics. Springer, Berlin Heidelberg New York, 2004.

[Mel99] R.E. Melchers. Structural reliability analysis and prediction. Wiley \& Sons, New York, 1999.

[MG97] R. Macklin and K. Garry. Assessing the effects of shear and turbulence during the dynamic testing of the crosswind sensitivity of road vehicles. Paper 970135, SAE (Society of Automotive Engineers), 1997.

[MGB02] G. Matschke, M. Grab, and B. Bergander. Nachweis der Sicherheit im Schienenverkehr bei extremen Seitenwind. ETR, Eisenbahntechnische Rundschau, 51(4), 2002.

[MGH02] R.L. Maon, R.F. Gunst, and J.L Hess. Statistical design and analysis of experiments. Wiley \& Sons, New York, 2002.

[MH02] G. Matschke and C. Heine. Full scale tests on side wind effects on trains. In B. Schulte-Werning et al., editors, TRANSAERO. Springer, Berlin Heidelberg New York, 2002.

[MKL86] H.O. Madsen, S. Krenk, and N.C. Lind. Methods of Structural Safety. Prentice-Hall, Englewood Cliffs, USA, 1986.

[MP04] S. Mansor and M.A. Passmore. Estimation of bluff body transient aerodynamics using an oscillating model rig. In Proc. BBAA $V$, 5th International Colloquium on Bluff Body Aerodynamics and Applications, July 11-15, Ottawa, Canada, 2004.

[MR97] W.O. Martinsen and T. Rahn. ICE Zug der Zukunft. Hestra, Darmstadt, 1997. 
[NS76] H. Neppert and R. Sanderson. Böenbeeinflussung schnellfahrender Züge. Z. Flugww., 24(3):151-161, 1976.

[OSB02] A. Orellano, M. Schober, and C. Bußmann. On side-wind stability of highspeed trains. In H.A. Mang, F.G. Rammerstorfer, and J. Eberhardsteiner, editors, Proc. of WCCM V, Fifth World Congress on Computational Mechanics, July 7-12, Vienna, Austria, 2002.

[OSR98] D.R. Oakley, R.H. Sues, and G.S. Rhodes. Performance optimization of multidisciplinary mechanical systems subject to uncertainties. Prob. Engn. Mechanics, 13:15-26, 1998.

[Pap02] A. Papoulis. Probability, random variables, and stochastic processes. McGraw-Hill, New York, 2002.

[PG02] R.C. Penmetsa and R.V. Grandhi. Efficient estimation of structural reliability for problems with uncertain intervals. Computers and Structures, 80:1103-1112, 2002.

[PK04] T. Payer and H. Küchenhoff. Modeling extreme wind speeds at a german weather station as basic input for a subsequent risk analysis for high-speed trains. J. of Wind Eng. and Ind. Aerodynamics, 92:241-261, 2004.

[PRI01] M.A. Passmore, S. Richardson, and A. Imam. An experimental study of unsteady vehicle aerodynamics. Journal of Automobile Engineering - Proc. Instn Mech. Engrs Part D, 215:779-788, 2001.

[PSP93] A. Parkinson, C. Sorensen, and N. Pourhassan. A general approach for robust optimal design. J. of Mechanical Design, 115:74-80, 1993.

[Rac01] R. Rackwitz. Reliability analysis - a review and some perspectives. Structural Safety, 23:365-395, 2001.

[Rai02] Rail Safety and Standards Board (RSSB), London. Railway safety and the ethics of the tolerability of risk, 2002.

[RE93] W. Rulka and A. Eichberger. SIMPACK - An analysis and design tool for mechanical systems. In W. Kortüm and R.S. Sharp, editors, Multibody Computer Codes in Vehicle System Dynamics, volume 22 of Supplement to Vehicle Sytem Dynamics. Swets and Zeitlinger B.V., Amsterdam and Lisse, 1993.

[RR01] Vents traversiers - Le TGV Méditerranée: performance et sécurité. Rail \& Recherche, 20:9-19, 2001.

[RRW04] C. Rolèn, T. Rung, and D. Wu. Computational modeling of cross-wind stability of high-speed trains. In Proc. of ECCOMAS 2004, July 24-28, Jyväskylä, Finland, 2004.

[Rub81] R.Y. Rubinstein. Simulation and the Monte Carlo Method. Wiley \& Sons, New York, 1981. 


\section{Bibliography}

[Rus82] H. Ruscheweyh. Dynamische Windwirkung an Bauwerken. Bauverlag, Wiesbaden Berlin, 1982 .

$\left[\mathrm{S}^{+} 01\right] \quad$ T.W. Simpson et al. Metamodels for computer-based engineering design: survey and recommendations. Engineering with Computers, 17:129-150, 2001.

$\left[\mathrm{S}^{+} 04\right] \quad \mathrm{S}$. Sanquer et al. Effects of cross winds on high-speed trains: development of a new experimental methodology. J. of Wind Eng. and Ind. Aerodynamics, 92:535-545, 2004.

[Sac83] D. Sachs. Methode zur Vorausberechnung der Sicherheit und der Laufgüte von Eisenbahnfahrzeugen bei Seitenwind und überlagerten Richtungsfehlern. ZEV - Glasers Annalen, 6:181-188, 1983.

[Sai02] M. Saito. Japanese railway safety and the technology of the day. Japan Railway \& Transport Review, 33:4-13, 2002.

[Sca97] R.H. Scanlan. Some observation on the state of bluff-body aeroelasticity. J. of Wind Eng. and Ind. Aerodynamics, 69-71:77-90, 1997.

[Sch97] W. Schiehlen. Multibody system dynamics: Roots and perspectives. Multibody System Dynamics, 1:149-188, 1997.

[Sch01] J.A. Schetz. Aerodynamics of high-speed trains. Ann. Rev. Fluid. Mech., 33:371-414, 2001.

[Sha89] A.A. Shabana. Dynamics of multibody systems. Wiley \& Sons, New York, 1989.

[SHB83] G. Schuëller, H. Hirtz, and G. Booz. The effects of uncertainties in wind load estimation on reliability assessments. J. of Wind Eng. and Ind. Aerodynamics, 14:15-26, 1983.

[SLC01] T.W. Simpson, D.K.J. Lin, and W. Chen. Sampling strategies for computer experiments: design and analysis. Int. J. of Reliability and Applications, 2(3):209-240, 2001.

[SNMG99] G. Schupp, H. Netter, L. Mauer, and M. Gretzschel. Multibody system simulation of railway vehicles with SIMPACK. Vehicle System Dynamics Supplement, 31:101-109, 1999.

[Sob01] I.M. Sobol. Global sensitivity indices for nonlinear mathematical models and their Monte Carlo estimates. Mathematics and Computers in Simulation, 55:271-280, 2001.

[Soc84] H. Sockel. Aerodynamik der Bauwerke. Vieweg, Braunschweig, 1984.

[Soc96] H. Sockel. Aerodynamics of trains. In J.A. Schetz and A.E. Fuhs, editors, Handbook of Fluid Dynamics and Fluid Machinery, pages 1721-1741. Wiley \& Sons, New York, 1996.

[SS96] E. Simiu and R.H. Scanlan. Wind Effects on Structures. Wiley \& Sons, New York, 1996. 


\section{Bibliography}

[SS98] R. Sigbjiörnsson and T. Snæbjörnsson. Probabilistic assessment of wind related accidents of road vehicles: A reliability approach. J. of Wind Eng. and Ind. Aerodynamics, 74-76:1079-1090, 1998.

[STM03] M. Suzuki, K. Tanemoto, and T. Maeda. Aerodynamic characteristics of train/vehicles under cross winds. J. of Wind Eng. and Ind. Aerodynamics, 91:209-218, 2003.

$\left[\mathrm{SW}^{+} 02\right]$ B. Schulte-Werning et al., editors. TRANSAERO: a European initiative on transient aerodynamics for railway system optimisation. Springer, Berlin Heidelberg New York, 2002.

[WH04] J. Wiedemann and W.H. Hucho, editors. Unsteady flow effects. Expert, Renningen (Germany), 2004.

[Wic03] A.H. Wickens. Fundamentals of Rail Vehicle Dynamics. Swets \& Zeitlinger, Lisse, The Netherlands, 2003.

[Wil97] E. Willemsen. High Reynolds number wind tunnel experiments on trains. $J$. of Wind Eng. and Ind. Aerodynamics, 69-71:437-447, 1997.

[WPO95] P.H. Wirsching, T.L. Paez, and K. Ortiz. Random vibrations. Wiley \& Sons, New York, 1995.

[WSH95] S. Watkins, J.W. Saunders, and P.H. Hoffmann. Turbulence experienced by moving vehicles. Part I. Introduction and turbulence intensity. J. of Wind Eng. and Ind. Aerodynamics, 57:1-17, 1995. 